\title{
Manufacture and Characterization of Ionic Polymer Transducers Employing Non-Precious Metal Electrodes
}

\author{
by \\ Matthew Bennett \\ Thesis submitted to the Faculty of the \\ Virginia Polytechnic Institute and State University \\ in partial fulfillment of the requirements for the degree of
}

\section{MASTER OF SCIENCE}

in

\section{Mechanical Engineering}

Donald J. Leo, Chair

Mark R. Anderson

Timothy E. Long

Douglas J. Nelson

May 2002

Blacksburg, Virginia

Keywords: ionic polymer, electroactive polymer, artificial muscle, Nafion ${ }^{\mathrm{TM}}$

Copyright by Matthew D. Bennett, 2002 


\section{Abstract}

Ionic polymer membranes are commonly used in fuel cell power generation, water electrolysis and desalinization, chlorine generation, and other niche applications. Since the early 1990s ionic polymer membranes have also shown promise as distributed electromechanical actuators and sensors. The cost of these materials is very high because of the expensive noble metals that are used as the electrodes in these applications, however. Currently, high cost of these devices has prevented them from experiencing widespread use. The goal of the current research project is to study new methods of plating metal electrodes onto ionic polymer membranes in order to reduce the cost of these materials and open the door for potential industrial, aerospace, and biomedical applications.

At this time ionic polymer actuators are only made using gold or platinum as the electrode in a lengthy and labor-intensive process. The current research focuses on using less costly metals and revising the metal deposition process. Several new methods allowing for faster deposition of metals onto ionic polymer membranes are developed and evaluated including sputter-coating, electroless plating, and impregnation/reduction. Using these methods, metal electrodes have been plated onto ionic polymer membranes in processes resulting in a purely surface deposition and in processes resulting in interpenetration of the metal into the polymer. This work shows that electromechanical coupling is present with all of these processes, although results indicate that interpenetration of the electrode is important for good adhesion of the metal and good performance of the transducers.

Also studied were different metals; X-ray photoelectron spectroscopy (XPS) testing shows that the use of non-noble metals as the electrodes results in oxidation of the metal and corresponding loss of performance in the actuator. Noble metals are found to not experience the oxidation problem. Further work shows that non-noble metals can be effectively employed as electrodes if alloyed with noble metals by using a co-reduction technique. Also 
studied is the use of protective coatings of noble metal to stabilize the non-noble metal electrodes. Using these approaches, a new plating method is developed and the stability of the electrodes made using this method is studied. These results indicate that samples made using this new process may be actauted continuously for over 150,000 cycles with very little degredation in their performance.

Using this new plating method, ionic polymer membrane transducers can be made in less than five hours. Characterization of these new devices shows that they have a mass energy density of 4-20 mJ/kg in the cantilevered mode. This compares well with a baseline material, which is found to have a mass energy density of 3-12 $\mathrm{mJ} / \mathrm{kg}$. Composition and morphology of the electrodes made using the new method are investigated using scanning electron microscopy (SEM) and the density and tensile modulus are measured. The density of the new material is found to be approximately $2100 \mathrm{~kg} / \mathrm{m}^{3}$ as compared to about 3200 $\mathrm{kg} / \mathrm{m}^{3}$ for the baseline material. Also, the tensile elastic modulus of the new material is about $55 \mathrm{MPa}$, or roughly one fourth of the tensile modulus of the baseline material (about $190 \mathrm{MPa}$ ). These results indicate that the new materials contain much less noble metal in the electrodes than the baseline material. The sensitivity of these devices has also been quantified and compared to the baseline. Results indicate that the new materials have a sensitivity on the order of $0.1-0.3 \frac{\mu \mathrm{A}}{\mathrm{mm} / \mathrm{s}}$ whereas similarly sized samples of the baseline material typically have sensitivities on the order of $0.2-0.8 \frac{\mu \mathrm{A}}{\mathrm{mm} / \mathrm{s}}$. The most important conclusion of this work is that ionic polymer membrane transducers can be made using much less noble metal in the electrode than previously believed without sacrificing the performance of these devices. 


\section{Acknowledgements}

The author would like to thank the following people for their assistance and understanding during this work:

- Dr. Donald Leo for believing in me enough to allow me to take on such a challenging project and to actually pay me for it.

- Dr. Mark Anderson, Dr. Tim Long, and Dr. Doug Nelson for valuable conversations and advice.

- Dr. Mohsen Shahinpoor and Dr. Kwang Kim of the University of New Mexico for donating the baseline Nafion ${ }^{\mathrm{TM}}$ polymer actuators used in this work.

- Dr. Alison Flatau of the National Science Foundation for supporting this work under Grant No. 9975678.

- The National Science Foundation for supporting the scanning electron microscope used in this work under Grant No. CMS 0093889.

- Mr. Kenn Newbury, Mr. Kevin Farinholt, Mr. Curt Kothera, Mr. Barbar Akle, Mr. Orion Parrott, Mr. Sean Brooks, Mr. Kiran Mallavarapu, and Ms. Alyssa Nicolaisen for their assistance and advice during this project.

- the rest of the CIMSS crew for tolerating my strange eating habits and especially my unmerciful sarcasm. 


\section{Contents}

List of Tables $\quad$ vii

List of Figures viii

Chapter 1 Introduction 1

1.1 History of Ionic Polymer Transducers . . . . . . . . . . . . . . . . . . 1

1.2 Background of Plating Methods for Ionic Polymer Transducers . . . . . . . 4

1.2.1 Mechanical and Electrical Deposition Methods . . . . . . . . . 5

1.2.2 Chemical Deposition Methods . . . . . . . . . . . . 6

1.3 Motivation for Research . . . . . . . . . . . . . . 8

1.4 Research Goals . . . . . . . . . . . . . . . . . . . 9 9

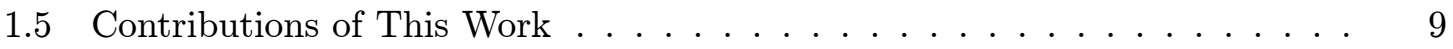

1.6 Overview of This Thesis . . . . . . . . . . . . . . . . 10

Chapter 2 Experimental Methods and Metrics $\quad 11$

2.1 Introduction . . . . . . . . . . . . . . . 11

2.2 Characteristics of Ionic Polymer Transducers _ . . . . . . . . . . . . 11

2.3 Overview of Metrics . . . . . . . . . . . . . . . . . 14

2.3.1 Surface Conductivity Measurements . . . . . . . . . . . 14

2.3.2 Force-Deflection Measurements . . . . . . . . . . . . . 14

2.3.3 Sensing Measurements . . . . . . . . . . . . . . . . . 17

2.3.4 Longevity Measurements . . . . . . . . . . . . . . . . . 18

2.3.5 Modulus Measurements . . . . . . . . . . . . . 20

2.3.6 Density Measurements . . . . . . . . . . . . . . 20

2.3.7 X-ray Photoelectron Spectroscopy _ . . . . . . . . . . . . . . 22 
2.3.8 Scanning Electron Microscopy . . . . . . . . . . . . . . . . . 23

Chapter 3 Description and Results of Initial Plating Methods 24

3.1 Introduction . . . . . . . . . . . . . . . . . . . . . . 24

3.2 Description of Baseline Material _ . . . . . . . . . . . . 25

3.3 Gold Sputter-Coating . . . . . . . . . . . . . . 26

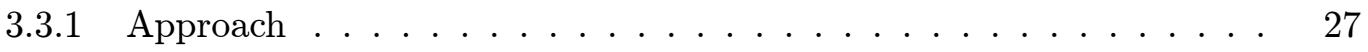

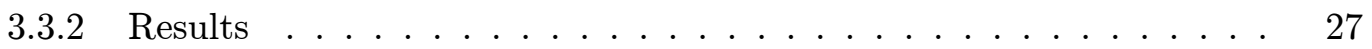

3.4 Electroless Nickel Plating . . . . . . . . . . . . . . . . . . . . . 32

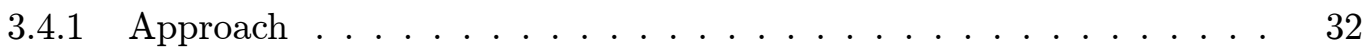

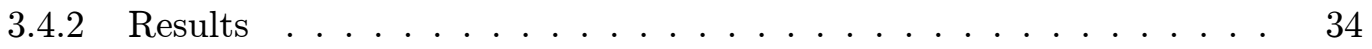

3.5 Impregnation/Reduction of Copper . . . . . . . . . . . . . . . . . 39

$3.5 .1 \quad$ Approach ............................ 41

3.5 .2 Results ............................. 44

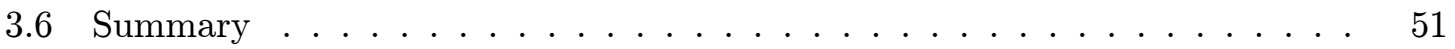

Chapter 4 Description and Results of Co-Reduction Method 54

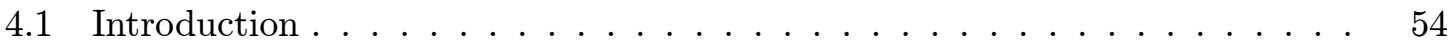

4.2 Concept of Co-Reduction $\ldots \ldots \ldots \ldots \ldots$

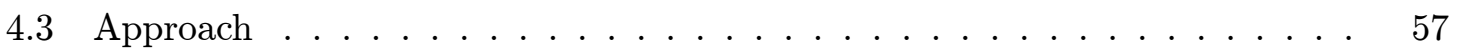

4.4 Details of New Process . . . . . . . . . . . . . . . . . . . 60

4.5 Characterization of New Process . . . . . . . . . . . . . . . . 69

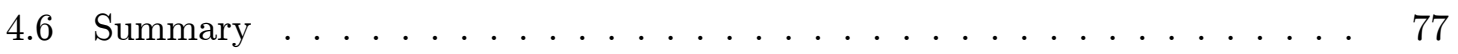

$\begin{array}{llr}\text { Chapter } 5 \text { Conclusions } & \mathbf{8 0}\end{array}$

5.1 Key Results and Conclusions . . . . . . . . . . . . . . . . 80

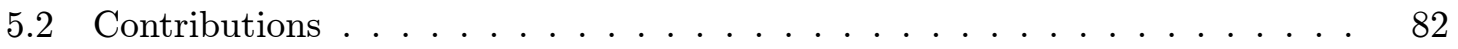

5.3 Suggestions for Future Work f . . . . . . . . . . . . . 83

$\begin{array}{lr}\text { Bibliography } & 85\end{array}$ 


\section{List of Tables}

4.1 Sensitivity coefficients of $5 \mathrm{~mm}$ wide strips of Nafion ${ }^{\mathrm{TM}}-117$ and Nafion ${ }^{\mathrm{TM}}$ 115 plated using the new process and clamped in a cantilevered configuration with a $17 \mathrm{~mm}$ free length. All data are in $\frac{\mu \mathrm{A}}{\mathrm{mm} / \mathrm{s}} \ldots \ldots \ldots$

4.2 Measured densities of several strips of Nafion ${ }^{\mathrm{TM}}-117$ and Nafion ${ }^{\mathrm{TM}}-115$ plated using the current method. All data are in $\mathrm{kg} / \mathrm{m}^{3}$. . . . . . . . . . . . . . 74

4.3 Measured peak blocked force, peak free deflection, and resulting peak energy densities of five strips of Nafion ${ }^{\mathrm{TM}_{-}}-117$ plated using the new process. Each strip was $5 \mathrm{~mm}$ wide with a $17 \mathrm{~mm}$ free length and the input was a $1.22 \mathrm{~V}$

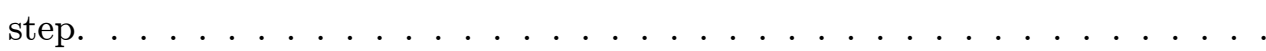

4.4 Measured peak blocked force, peak free deflection, and resulting peak energy densities of five strips of Nafion ${ }^{\mathrm{TM}_{-}}-115$ plated using the new process. Each strip was $5 \mathrm{~mm}$ wide with a $17 \mathrm{~mm}$ free length and the input was a $1.22 \mathrm{~V}$ step. . . . . . . . . . . . . . . . . . .

4.5 Measured elastic modulus of several strips of Nafion ${ }^{\mathrm{TM}}-117$ and Nafion ${ }^{\mathrm{TM}}$ 115 plated using the current method. All data are in MPa. . . . . . . . . 


\section{List of Figures}

1.1 The chemical structure of the Nafion ${ }^{\mathrm{TM}}$ polymer. . . . . . . . . . 2

1.2 An ionic polymer membrane as (a) an actuator, (b) a sensor. . . . . . . . 4

2.1 Blocked force response of an ionic polymer actuator to a $1.22 \mathrm{~V}$ step input. 13

2.2 Free deflection response of an ionic polymer actuator to a $1.22 \mathrm{~V}$ step input. Note some resonance in the response when the step is applied. . . . . . . . 13

2.3 (a) Photo of the setup used to perform the force and deflection tests. (b) Detail of the test fixture. . . . . . . . . . . . . . . . . 16

2.4 Schematic of the setup used to perform the sensing tests. . . . . . . . 18

2.5 Photo of the setup used to perform the longevity testing. . . . . . . . . . 19

2.6 (a) Photo of the setup used to perform the modulus testing. (b) Detail of the test fixture. . . . . . . . . . . . . . . . . .

3.1 Photos of samples of Nafion ${ }^{\mathrm{TM}}$ membrane sputtered with gold for (a) one, (b) two, (c) three, and (d) four coating cycles. . . . . . . . . . . . 28

3.2 Measured surface resistance of the sputtered gold coating as a function of

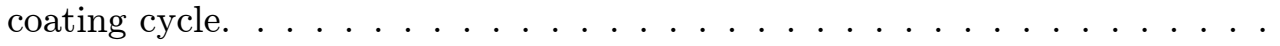

3.3 Scanning electron micrographs of the surface of samples of Nafion ${ }^{\mathrm{TM}}$ that were sputter-coated with gold for (a) one sputtering cycle, (b) two sputtering cycles, (c) three sputtering cycles, and (c) four sputtering cycles. . . . . . .

3.4 Free tip deflection versus time of a sample sputtered with gold for two sputtering cycles. The sample was $5 \mathrm{~mm}$ wide with a $20 \mathrm{~mm}$ free length and the input was a $1.5 \mathrm{~V}$ square wave. . . . . . . . . . . . . 
3.5 Blocked force response to a $4.0 \mathrm{~V}$ step input of a strip of Nafion ${ }^{\mathrm{TM}}$ that had been coated with gold for one sputtering cycle. This sample was $5 \mathrm{~mm}$ wide with a $20 \mathrm{~mm}$ free length. . . . . . . . . . . . . . .

3.6 (a) Blocked force response of a nickel-plated Nafion ${ }^{\mathrm{TM}}$ membrane to a $3.0 \mathrm{~V}$ input, (b) Blocked force response of the baseline material to a $1.5 \mathrm{~V}$ input. Both samples were $5 \mathrm{~mm}$ wide with a $20 \mathrm{~mm}$ free length. . . . . . . . . . 35

3.7 Blocked force response of a nickel-plated sample to a $3.0 \mathrm{~V}$ step input. The sample was $5 \mathrm{~mm}$ wide with a $20 \mathrm{~mm}$ free length. . . . . . . . . . . 36

3.8 Photo of the tension fixture, with a membrane sample mounted in place. . . $\quad 37$

3.9 Photo of a sample plated with nickel without using the plating fixture. Note that the membrane has become wrinkled and creased during the plating process. 37

3.10 Measured XPS data of a nickel-plated sample that had experienced some corrosion of the nickel electrodes. . . . . . . . . . . . . . . . . . 39

3.11 Reference XPS spectra of nickel and nickel oxide (60). . . . . . . . . . . . 40

3.12 Photo of a sample of Nafion ${ }^{\mathrm{TM}}$ membrane plated with copper. . . . . . . 43

3.13 (a) Photo of the fixture in a clamp, with a template used to cut the membrane to the correct size. (b) Photo of a membrane sample in the fixture . . . . . 44

3.14 Force/deflection curve for a sample of Nafion ${ }^{\mathrm{TM}} 5 \mathrm{~mm}$ wide with a $17 \mathrm{~mm}$ free length that was plated with copper with a gold overlayer. The input was a $1.0 \mathrm{~V}$ step and the test was performed immediately after plating. Note that the plot was generated using only the blocked force and free deflection.

3.15 Scanning electron micrograph showing the penetrating copper layer and the gold overlayer. Note that the copper layer is about 0.6 microns thick. . . . .

3.16 Micrograph showing that the gold layer is about 50 nanometers thick. The copper layer is not visible due to the low contrast of the image. . . . . . . 46

3.17 Measured XPS spectrum of the surface of a copper-plated sample that had undergone some corrosion. . . . . . . . . . . . . . . . . 47

3.18 Reference XPS spectra of copper and copper oxide (60) . . . . . . . . 48

3.19 Photos of samples of Nafion ${ }^{\mathrm{TM}}$ membrane plated with copper with a gold overlayer (a) before oxidation of the copper layer, and (b) after oxidation of the copper layer. . . . . . . . . . . . . . . . . 
3.20 Normalized tip displacement versus the number of actuation cycles of a strip of Nafion ${ }^{\mathrm{TM}}$ plated with copper, and a strip plated with copper with a gold overlayer. The input was a $1.5 \mathrm{~V}, 1.0 \mathrm{~Hz}$ sine wave. . . . . . . . . . .

3.21 Normalized tip displacement versus the number of actuation cycles of four strips of Nafion ${ }^{\mathrm{TM}}$ plated with copper with a gold overlayer. The input was a 1.25 V, $1.0 \mathrm{~Hz}$ sine wave. Nitrogen gas was bubbled through the water bath for 2 hours prior to testing and the space above the bath was flushed with nitrogen during testing. . . . . . . . . . . . . . .

3.22 Normalized tip displacement versus the number of actuation cycles of three strips of Nafion ${ }^{\mathrm{TM}}$ plated with copper with a gold overlayer. The input was a $1.25 \mathrm{~V}, 1.0 \mathrm{~Hz}$ sine wave. Nitrogen gas was bubbled through all solutions during the plating process. The samples were actuated in a bath of mineral

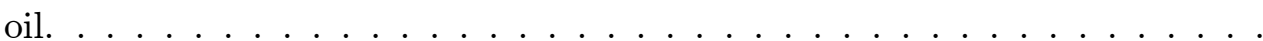

4.1 Normalized tip displacement versus the number of actuation cycles of two strips of Nafion ${ }^{\mathrm{TM}}$ plated with an alloy of copper and gold. The input was a $1.25 \mathrm{~V}, 1.0 \mathrm{~Hz}$ sine wave. . . . . . . . . . . . . . . . . .

4.2 Normalized tip displacement versus the number of actuation cycles of a strip of Nafion ${ }^{\mathrm{TM}}$ deposited with gold and then with copper and subsequently electroless plated with an overlayer of gold. The input was a $3.0 \mathrm{~V}, 1.0 \mathrm{~Hz}$ sine wave. . . . . . . . . . . . . . . . . . .

4.3 (a) Scanning electron micrographs of the cross-section of a sample plated using a 50\% copper, 50\% platinum plating solution. (b) Close-up of the left electrode. . . . . . . . . . . . . . . . . . .

4.4 (a) Scanning electron micrographs of the cross-section of a sample plated using a $80 \%$ copper, $20 \%$ platinum plating solution. Note that the damage near the right edge was caused by the X-ray source. (b) Close-up of the left electrode. . . . . . . . . . . . . . . . . .

4.5 Normalized tip displacement versus the number of actuation cycles for three sets of two samples each plated with a copper/platinum alloy electrode using different ion-exchange solutions. The input was a $1.25 \mathrm{~V}, 1.0 \mathrm{~Hz}$ sine wave. 
4.6 Measured atomic fraction of platinum metal in electrodes versus ionic fraction of tetraammine platinum ion in exchanging solution. This data was obtained by averaging the results of several X-ray scans for each sample. . . . . . . .

4.7 (a) Scanning electron micrographs of the cross-section of a sample plated using a $90 \%$ copper, $10 \%$ platinum plating solution. (b) Close-up of the left

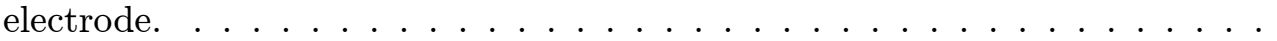

4.8 (a) Scanning electron micrographs of the cross-section of a sample plated using a $95 \%$ copper, $5 \%$ platinum plating solution. (b) Close-up of the left electrode. Note that the gold overlayer is visible in these images. . . . . . .

4.9 Photos of samples of Nafion ${ }^{\mathrm{TM}}$ processed using the new method (a) before deposition of the gold layer, (b) after deposition of the gold layer. . . . . . .

4.10 Scanning electron micrograph showing a close-up of the gold overlayer on a sample plated with a copper/platinum alloy and subsequently with gold. Note that the gold layer is approximately 50-80 nanometers thick. . . . . . 71

4.11 Normalized tip displacement versus the number of actuation cycles for a sample plated using an exchanging solution of 95\% copper ion and 5\% tetraammine platinum ion with a protective gold layer. The input was a $1.25 \mathrm{~V}, 1.0$

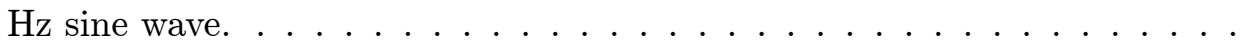

4.12 Sensing response versus frequency of a sample of Nafion ${ }^{\mathrm{TM}}-117$ plated with a copper/platinum alloy and a gold overlayer. The average sensitivity over the indicated frequency range is $0.1075 \frac{\mu \mathrm{A}}{\mathrm{mm} / \mathrm{s}} \ldots \ldots \ldots \ldots$

4.13 Normalized tip displacement versus the number of actuation cycles for a sample of Nafion ${ }^{\mathrm{TM}}-117$ plated using the new process. The input was a 1.25 $\mathrm{V}, 1.0 \mathrm{~Hz}$ sine wave. . . . . . . . . . . . . . . . 


\section{Chapter 1}

\section{Introduction}

\subsection{History of Ionic Polymer Transducers}

Ionic polymers are materials that exhibit ion selectivity due to their molecular structure. This ionic selectivity makes ionic polymers useful in many applications, including water electrolyzers, solid electrolyte fuel cells, chlorine and other gas generators, and other electrochemical processors. In the late 1940s it was discovered that ionic polymers could also be used as electrochemomechanical actuators. The pioneering work in this field was done by W. Kuhn et al. of the University of Basle (Switzerland) and A. Katchalsky et al. of the Weizmann Institute of Science (Israel) $(21 ; 22 ; 18)$. These researchers showed that a polymer made by copolymerizing methacrylic acid with divinyl benzene would swell and dialate upon changes to the $\mathrm{pH}$ of the surrounding solution, effectively making a chemomechanical actuator. In 1965 Hamlen et al. extended this idea to demonstrate an electromechanical ionic polymer actuator (17). By impregnating a fiber of a copolymer of polyvinly alcohol and polyacrylic acid (supplied by Katchalsky) with platinum, they were able to suspend the fiber in a liquid electrolyte and pass an electric current through the electrolyte to the fiber. The change in $\mathrm{pH}$ resulting from the passage of this electric current caused the same swelling and dialation observed by Kuhn and Katchalsky in their early work. In 1973 Yannas and Grodzinsky did a similar experiment using collagen (a natural polymer electrolyte) fibers suspended in a liquid electrolyte (62). Again, mechanical actuation was demonstrated in response to an electrical input. In 1974 and 1975, Grodzinsky and Melcher followed this work by demonstrating that a deformable collagen membrane could produce mechanical motion when activated by an electrical input in a liquid electrolyte $(13 ; 14)$. More im- 
portantly, they also showed that a voltage drop was produced across the membrane when it was acted on by an external mechanical input, demonstrating the ability of a polymer electrolyte membrane to act not only as an actuator, but also as a sensor.

The most common ionic polymer in use today is DuPont's Nafion ${ }^{\text {TM }}$. Developed in the 1960's and commercialized in the 1970's, it has found a myriad of applications since then. Nafion ${ }^{\mathrm{TM}}$ is a telfon-based polymer with pendant sulfonic acid side groups. DuPont reports the acid capacity of Nafion ${ }^{\mathrm{TM}}$ to be 0.89 moles of sulfonate exchange sites per gram of dry polymer and its equilibrium water content to be $5 \%$ of its dry weight in water at $23{ }^{\circ} \mathrm{C}, 50 \%$ relative humidity (11). These acidic sidegroups dissolve when the material is hydrated by boiling in water, causing the membrane to swell significantly, absorbing as much as $38 \%$ of its dry weight in water and causing a linear expansion of as much as $15 \%$ (11). This dissolution frees the cation associated with each pendant acidic group to move within the polymer matrix while the anion maintains a bond to the fluorocarbon backbone. It is this property of Nafion ${ }^{\mathrm{TM}}$ membranes which allows them to transmit cations while blocking anions and makes them useful as selective ion exchange membranes in chlor-alkali processes, hydrogen-air fuel cells, and the like.

The chemical structure of a Nafion ${ }^{\mathrm{TM}}$ repeating unit is shown in Figure 1.1 below (35). In this figure, $\mathrm{b}$ is probably 1 , a varies from 5 to 11 , and $\mathrm{M}^{+}$is the mobile cation (64; 49). Yeager $(63 ; 66)$, Gierke and Hsu (12), and Nemat-Nasser and Li (35) report that the Nafion ${ }^{\mathrm{TM}}$ polymer consists of hydrophilic "clusters" surrounded by hydrophobic regions. These hydrophilic clusters contain the sulfonate exchange sites and the mobile cations and are interconnected by narrow channels through which the cations may pass while the hydrophobic regions contain the fluorocarbon backbone. This result is very important

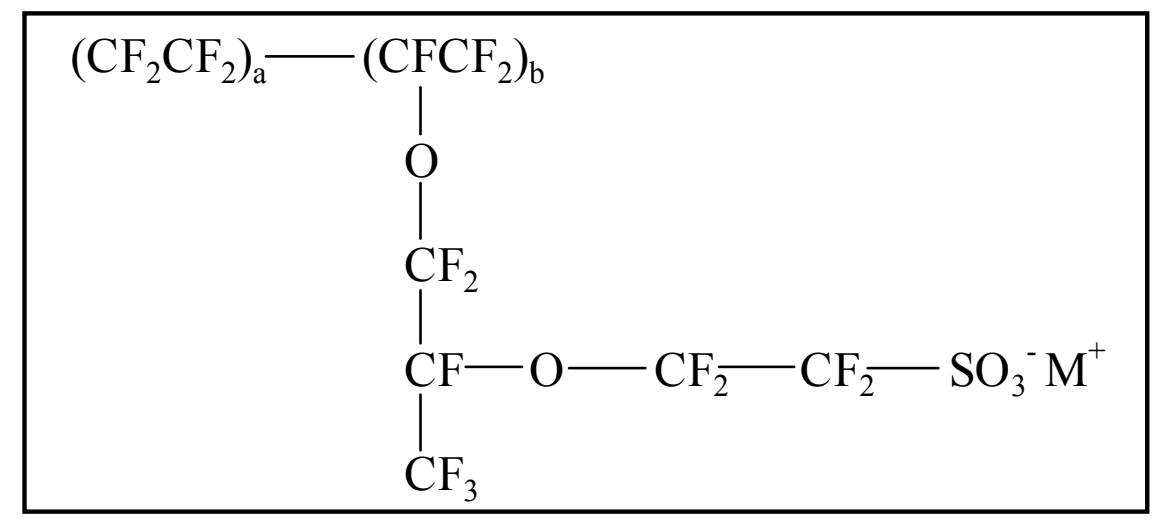

Figure 1.1: The chemical structure of the Nafion $^{\mathrm{TM}}$ polymer. 
to understanding the mechanism of ionic motion within the polymer and the resulting deformation of the membrane.

In recent years, Nafion ${ }^{\mathrm{TM}}$ membranes have been used as electromechanical actuators and sensors. In the early 1990s, three groups of researchers published papers that demonstrated the use of Nafion ${ }^{\mathrm{TM}}$ membranes as electromechanical transducers. In 1992, Sadeghipour, Salomon, and Neogi developed an accelerometer using a Nafion ${ }^{\mathrm{TM}}$ membrane (47). They demonstrated that the voltage produced by a small cantilever constructed from Nafion $^{\mathrm{TM}}$ was proportional to the acceleration. Thus, an electromechanical sensor was produced from an ion-exchange membrane. Almost concurrently with Sadeghipour et al., Oguro et al. and Segalman et al. published results demonstrating the use of Nafion ${ }^{\mathrm{TM}}$ based composites as electromechanical actuators $(42 ; 48)$. Both groups demonstrated that mechanical deformation is produced in the membrane when an electric field is applied to the material.

The principle of electromechanical transduction is shown in Figure 1.2. Figure 1.2a is a picture of a Nafion ${ }^{\mathrm{TM}}$ membrane that has been hydrated and coated with a conductive electrode on both sides. Upon application of an electric field, the membrane bends towards the anode due to the motion of the mobile cations and water molecules within the polymer matrix. Work by Mallavarapu and Leo has shown that feedback control may be used effectively to control the motion of these actuators in a free bender configuration $(28 ; 27)$. The converse property is exhibited in Figure 1.2b. A cantilever sample of an ion-exchange membrane has deformed by the application of a force to the tip of the sample. It is believed that macro motion of the membrane produces micro motion of the mobile cations, resulting in a charge imbalance across the electrodes. Several researchers have demonstrated that the quasi-static displacement of the polymer is correlated with the voltage that is produced by the membrane $(49 ; 34 ; 19)$, and more recently, Newbury and Leo demonstrated that the current induced in the membrane is proportional to the rate of motion caused by the mechanical deformation $(37 ; 36)$.

Since the early 1990s several other research groups have become involved with ionic polymer membranes as actuators. In 1993, Oguro et al. patented the notion of using ionic polymer membranes as actuators (39). Oguro et al. have also demonstrated novel applications of ionic polymer actuators, using them in active catheters (16), underwater microrobots (15), and as elliptical drive elements in rotary and linear motors (57). Bar- 


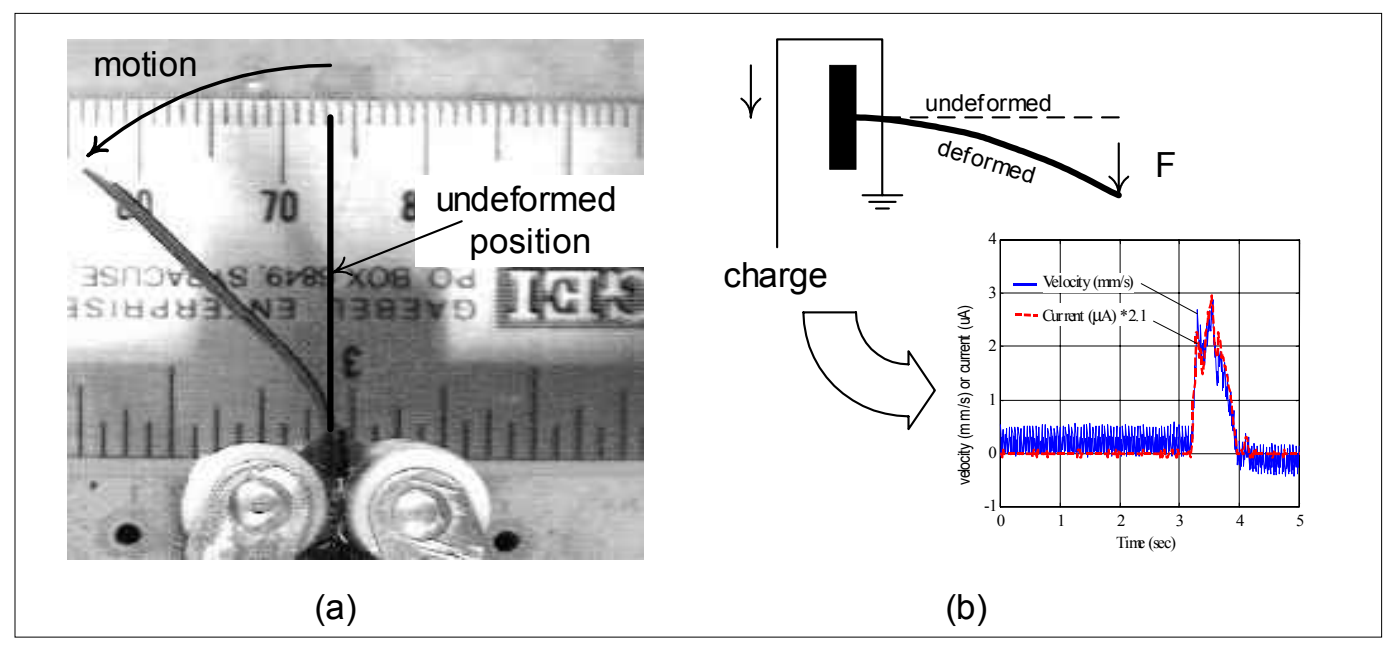

Figure 1.2: An ionic polymer membrane as (a) an actuator, (b) a sensor.

Cohen has focused more on the use of these devices for interplanetary applications, and has demonstrated their ability to operate as low-mass actuators for robotic grippers and dust wipers for camera lenses (4). Although these represent novel applications for ionic polymer actuators, the state of the current technology has limited the use of these materials to laboratory demonstrations.

Research has also focused on increasing the work output of these actuators. Shahinpoor has made several developments in this area, showing that more force can be obtained from ionic polymer actuators by increasing the conductivity of the electrode (53) and by decreasing the porosity of the electrode (20). Work by Shahinpoor et al. and Abe et al. has also shown that increased performance can be obtained by changing the mobile cation inside the membrane, with the best results obtained when lithium is used $(1 ; 52)$. Rashid and Shahinpoor have also performed experimental optimization of the plating process using a Taguchi method in order to improve the performance of Nafion ${ }^{\mathrm{TM}}$ transducers (46).

\subsection{Background of Plating Methods for Ionic Polymer Trans- ducers}

The principle of electromechanical transduction in ionic polymer membrane devices is based on the motion of hydrated mobile cations within the polymer matrix. This motion is induced by electrostatic forces which arise from the application of an electric field across the membrane thickness. Conversely, if the material is caused to bend by an applied external 
force, then an electric field may be generated across the thickness. In this way an ionic polymer membrane may function as both a distributed actuator and sensor. In order for an electric field to be generated across the thickness of an ionic polymer membrane, its surfaces must be made to conduct electricity. This is typically done by plating both sides of the membrane with a metallic electrode. This plating has typically been done using either a mechanical or a chemical deposition method. Most of this literature survey summarizes the development of plating methods for ionic polymer membranes that were to be used in electrochemical processes, for example, hydrogen-oxygen fuel cells, water electrolysis cells, $\mathrm{CO}_{2}$ reduction cells, chemical sensors, etc. Only since the early 1990's have researchers begun to focus on the development of plating methods for making electromechanical ionic polymer transducers.

\subsubsection{Mechanical and Electrical Deposition Methods}

Most of the efforts to coat ionic polymer membranes with metallic electrodes have been aimed at improving fuel cell technology by decreasing the required catalyst loading of the fuel cell membranes. This is primarily because the effective catalysts for the reverse electrolysis reaction that takes place at the membrane of a fuel cell are noble metals such as platinum, ruthenium, and iridium, which account for most of the cost of the cell. The following literature review will attempt to touch on the high points of the development of these plating processes, but this is not an exhaustive account of the development of fuel cell technology.

In 1981, Lawrance and Wood of the General Electric Company patented a method for depositing metal powders onto ionic polymer membranes using elevated temperature and pressure (23). They found that electrodes made by this method had significantly lower loadings of noble metals than those made using previously published methods while maintaining similar performance in water electrolysis cells. Iridium and platinum were used as the electrodes in their study. A similar method has been developed by Wilson and Gottesfeld of Los Alamos National Laboratory (USA) in which an ink is prepared that contains platinum particles suspended on particles of carbon, with a Nafion ${ }^{\mathrm{TM}}$ binder (61). This ink is spread onto a Teflon ${ }^{\mathrm{TM}}$ blank and dried. This blank is then hot-pressed onto an ionic polymer membrane and the Teflon ${ }^{\mathrm{TM}}$ blank is peeled away. In 2001, Surampudi et al. of the California Institute of Technology patented a process for forming a direct- 
methanol fuel cell that used a Nafion ${ }^{\mathrm{TM}}$ membrane as the electrolyte $(55 ; 56)$. In this work platinum and ruthenium are used as the catalytic electrodes and are applied to the Nafion $^{\mathrm{TM}}$ membrane either by sputtering or by painting directly onto the membrane from an ink containing submicron catalyst particles and Nafion ${ }^{\mathrm{TM}}$ and Teflon ${ }^{\mathrm{TM}}$ polymers as binders.

For the purpose of fabricating electromechanical actuators, the most popular electrode deposition method is based on chemical reduction, but mechanical plating methods have also shown promise. In 1999, Liu et al. of NanoSonic, Inc (USA) developed a process to plate gold onto ionic polymer membranes by electrostatic self-assembly (26). By alternatively depositing monolayers of an anionic polymer with monolayers of gold colloids suspended in a solution of a cationic polymer, a very thin $(85 \mathrm{~nm})$ electrode was acheived that had an electrical resistivity only about twice that of pure gold. In 2001, Zhou et al. published the results of a deposition process in which a seed layer of gold is initially deposited by sputter-coating (67). Following this initial layer, which is about 400 nanometers thick, more gold is deposited onto the membrane through electroplating. This second layer is approximately 2.25 microns thick and is needed to increase the conductivity of the electrode. Also in 2001, Shahinpoor and Kim reported on a technique whereby electrodes were plated onto Nafion ${ }^{\mathrm{TM}}$ membranes by first depositing fine silver powder from a suspension onto a backing material (51). This backing material is then pressed against the surface of the Nafion ${ }^{\mathrm{TM}}$ under elevated temperature and pressure to form an electrode structure on the Nafion ${ }^{\mathrm{TM}}$. Further plating of platinum is carried out by chemical reduction, followed by electroplating of gold or palladium.

\subsubsection{Chemical Deposition Methods}

Chemical plating methods for ionic polymer membranes are based on the chemical reduction of the metal to be plated from an aqueous solution. Typically, the ion-exchange properties of ionic polymers are used to facilitate the deposition of the metallic electrode. For this reason, the electrode formation can take place in the presence of aqueous solutions without the need for heat, pressure, or binders. It should be noted that the first chemical plating method reported for an ionic polymer device was done by Hamlen et al. in 1965 (17). In their work, a fiber of a copolymer of polyvinyl alcohol and polyacrylic acid was impregnated with platinum by alternating treatments of the fiber with platinic chloride and sodium 
borohydride. The platinum ions diffuse into the polymer from the platinic chloride and are subsequently reduced to the metallic state by the sodium borohydride. In 1982, Takenaka et al. published the results of their chemical deposition process (59). In this study, iridium, rhodium, platinum, ruthenium, and palladium metals and alloys of these metals were used as the electrodes and the ionic polymers electroded in this way were analyzed for their performance in water electrolysis cells. This process came to be known as the TakenakaTorikai process and was awarded a Japanese patent in 1980 (58). In the Takenaka-Torikai process the cationic reducing agent is allowed to diffuse through the membrane to react with an anionic salt of the metal to be plated. This process is effective because the anionic salt cannot diffuse through the Nafion ${ }^{\mathrm{TM}}$ membrane, thus the electrode is formed near the surface, a few microns into the polymer. In 1988, Millet, Pineri, and Durand developed a similar process for the plating of platinum onto Nafion ${ }^{\mathrm{TM}}$ membranes, which has been the basis of the most common modern plating methods (33). Millet later reported on a research effort to further study and optimize this process $(30 ; 31)$. The fundamental deviation from the Takenaka-Torikai process is that the metal is plated from a cationic salt. Using this "impregnation/reduction" process, a cation of the metal to be plated is incorporated into the membrane and subsequently reduced to its neutral state at the membrane surface by a reducing agent in aqueous solution. Because both of these processes result in a metal electrode that strongly interpenetrates the ionic polymer membrane, adhesion of the metal to the polymer is excellent. Also in 1988, Cook et al. (9) and DeWulf and Bard (10) reported on a similar process in which copper was plated onto Nafion ${ }^{\mathrm{TM}}$ membranes by diffusion of copper ions from one compartment of a two-compartment cell through the membrane to a reducing agent on the other side. Again, the purpose of this copper-plated membrane was not to make an electromechanical actuator, but rather was to perform the electrochemical reduction of carbon dioxide. In 1993 Chen and Chou plated copper and many other metals (platinum, silver, lead, and nickel, as well as alloys of these metals) onto Nafion ${ }^{\mathrm{TM}}$ membranes to study the catlytic effects of these metals in the electrochemical reduction of benzaldehyde (8). Several different plating methods were used in their study, including a diffusion method (similar to the Takenaka-Torikai method), an impregnation/reduction process (similar to the process used by Millet et al.), and an electroless plating method. 


\subsection{Motivation for Research}

The current research is motivated by the high cost of ionic polymer actuators and sensors. These materials, which function as distributed actuators and sensors, have the potential to be used in many industrial and research applications, but are limited by the high cost to fabricate them. Devices and materials that function as distributed actuators and sensors are commonly referred to as "smart" materials. Some examples of common smart materials are piezoelectric ceramics and polymers, shape memory alloys, and and magnetorheological fluids. Ionic polymer actuators and sensors represent a relatively new entry into the field of smart materials. Unlike many other smart materials, ionic polymers may undergo large displacements at relatively low voltages (1-5 V). They also exhibit relatively fast response (10's of $\mathrm{Hz})$ and are amenable to operation in aqueous environments. However, there are no commercial sources or applications for ionic polymer actuators or sensors at this time. One reason for this is that the technology is still very new and not well understood, but another reason is that the technology is still very expensive. A main reason for the high cost of these devices is the high cost of the precious metals that are typically used as electrodes.

From this study it is evident that most of the efforts to plate metals onto ionic polymer membranes have focused on the noble metals. In most of the above reviewed reports, the reason for this is that the catalytic nature of these metals is necessary for the specific application. Also, due to the hydrated and acidic nature of the polymer, non-noble metals may not be stable enough to avoid corrosion.

When using an ionic polymer membrane as a electromechanical actuator or sensor, no electrochemical reaction is taking place and therefore no catalyst is needed. For this reason, noble metals may not seem the best choice as an electrode metal for actuation and sensing applications. However, noble metals have typically been employed in these applications due to their inherent stability in corrosive environments. At this time, most ionic polymer actuators are made using gold or platinum as the electrode. While the use of noble metal electrodes has proven effective in ionic polymer trandsucer technology, the high cost of these metals ( $\sim 285$ per ounce for gold and $\sim \$ 480$ per ounce for platinum) have kept the price of these transducers relatively high and prevented them from being able to compete with other actuator technologies. 


\subsection{Research Goals}

The goal of the current research is to reduce the cost of ionic polymer membrane transducers by employing less expensive metals as the conductive electrodes. Also of interest is the investigation of new methods of plating the electrodes onto ionic polymer membranes in order to gain a greater understanding of how the electrode geometry and polymer/electrode interface affects the performance of these devices. By studying the use of less expensive metals and different ways of plating these metals to form electrodes, it is hoped that the key issues associated with the use of these metals and methods can be identified and strategies developed to overcome these issues.

\subsection{Contributions of This Work}

This thesis presents an overview of a research project aimed at improving the methods of plating electrodes onto ionic polymer membranes. Through the course of this work several different plating methods were developed and studied, including processes involving purely surface deposition of metals and methods involving interpenetration of the metal and the polymer. The benefits and drawbacks of each method were analyzed and the features of a successful plating method were identified. Several different metals were plated as electrodes and the issues associated with the use of each of these metals were identified. Based on the results with these processes a new process has been developed that involves significant reductions in the amount of noble metal required for deposition of stable electrodes. These new devices have been tested as actuators and sensors and their transduction properties compared to a baseline material supplied by Dr. Mohsen Shahinpoor of the University of New Mexico. Results indicate that ionic polymer transducers can be manufactured using this new processing method in less than five hours and that their properties compare favorably with the baseline material. This work has made significant advances towards

reducing the cost of fabricating ionic polymer membrane transducers and is the first step towards further study into improvements of this technology. 


\subsection{Overview of This Thesis}

The purpose of this thesis is to document the discovery of a new method of plating the electrodes onto ionic polymer membranes that reduces the cost of these electrodes. This document will be structured such that the discussion of the various plating methods studied is essentially chrolnological. Chapter 2 discusses the various performance metrics used to characterize ionic polymer sensors and actuators and the methods of measuring these metrics. In Chapter 3, the initial plating processes that were investigated are discussed, as well as the results of these plating methods and some conclusions regarding these results. Chapter 4 covers the details of the final plating process that was developed snd summarizes the results of testing done using this process. Finally, Chapter 5 presents the conclusions of this research and lists suggestions for future work in this area. 


\section{Chapter 2}

\section{Experimental Methods and Metrics}

\section{$2.1 \quad$ Introduction}

This chapter descibes some of the metrics that are used to quantify the performance of ionic polymer sensors and actuators and how these metrics are obtained. Included is detailed information on equipment used including manufacturer and model number as well as experimental procedures utilized. Also discussed are possible sources of experimental error. The discussion of these metrics and how they are relevant to ionic polymer transducers is important in order to understand how comparisons are made between materials fabricated using different processes.

\subsection{Characteristics of Ionic Polymer Transducers}

The ionic polymer membranes used in this study are made of DuPont's Nafion ${ }^{\mathrm{TM}}$, a form of sulfonated Teflon ${ }^{\mathrm{TM}}$ that is very stable in many environments. This polymer is hygroscopic, absorbing large amounts of water upon hydration (up to $38 \%$ by dry weight) (11). Mallavarapu and Leo have shown that the frequency response of the polymer trandsucers changes with changes in the water content, indicating that the response is a function of the hydration level (27). For this reason, all testing is performed with the polymer in a fully hydrated state. The hydration is maintained by either performing the testing with the polymer submerged in water, or in cases where the polymer is operated in air, water is frequenty 
applied to the polymer by brushing. However, Steck and Yeager have shown that Nafion ${ }^{\mathrm{TM}}$ membranes may attain different hydration states and that transitions between these states may be irreversible (54). These researchers explain that upon boiling, the Nafion ${ }^{\mathrm{TM}}$ polymer is fully hydrated and if this water is removed then the polymer must be boiled again in order to be returned to this fully hydrated state. They argue that another, less hydrated state is reached when room temperature $\left(27^{\circ} \mathrm{C}\right)$ water is added to the polymer. Therefore, if the ionic polymer transducer samples analyzed in this study dried out during the testing and were then re-hydrated by brushing with room temperature water, the water content of the polymer may have been changed as a result, thus changing the performance of the samples in an unpredictable way. This is one possible source of experimental error in this study.

Another interesting characteristic of ionic polymer transducers is their unique response to a step input. Upon application of an electric field to the device, a charge flow into the polymer is induced and the material suddenly bends towards the anode. It is believed that this bending is due to motion of the water molecules and free cations within the polymer. Once bent, pressure and concentration gradients are set up within the polymer membrane. These gradients drive the water molecules and free cations back towards their neutral positions, causing relaxation of the ionic polymer membrane, even with the field still applied. The response of the membrane to a step is then a quick rise to a peak value followed by a slow relaxation to a small steady-state value (see Figures 2.1 and 2.2). Smart material technologies are typically characterized by a force/deflection curve that represents the steady-state response of the material for a given input. Because the peak response of an ionic polymer actuator is so much larger than the steady-state response, the concept of a force-deflection curve for an ionic polymer actuator is a bit different than for these other technologies. The reported forces and deflections are the peak values measured in response to a step input, so they represent the maximum possible force or deflection an ionic polymer transducer can generate, not the steady-state value. These numbers can then be though of as the representing the work output of these devices if operated well below their resonance, but are not DC values. This is important to remember when making comparisons between ionic polymer actuators and other actuation technologies. 


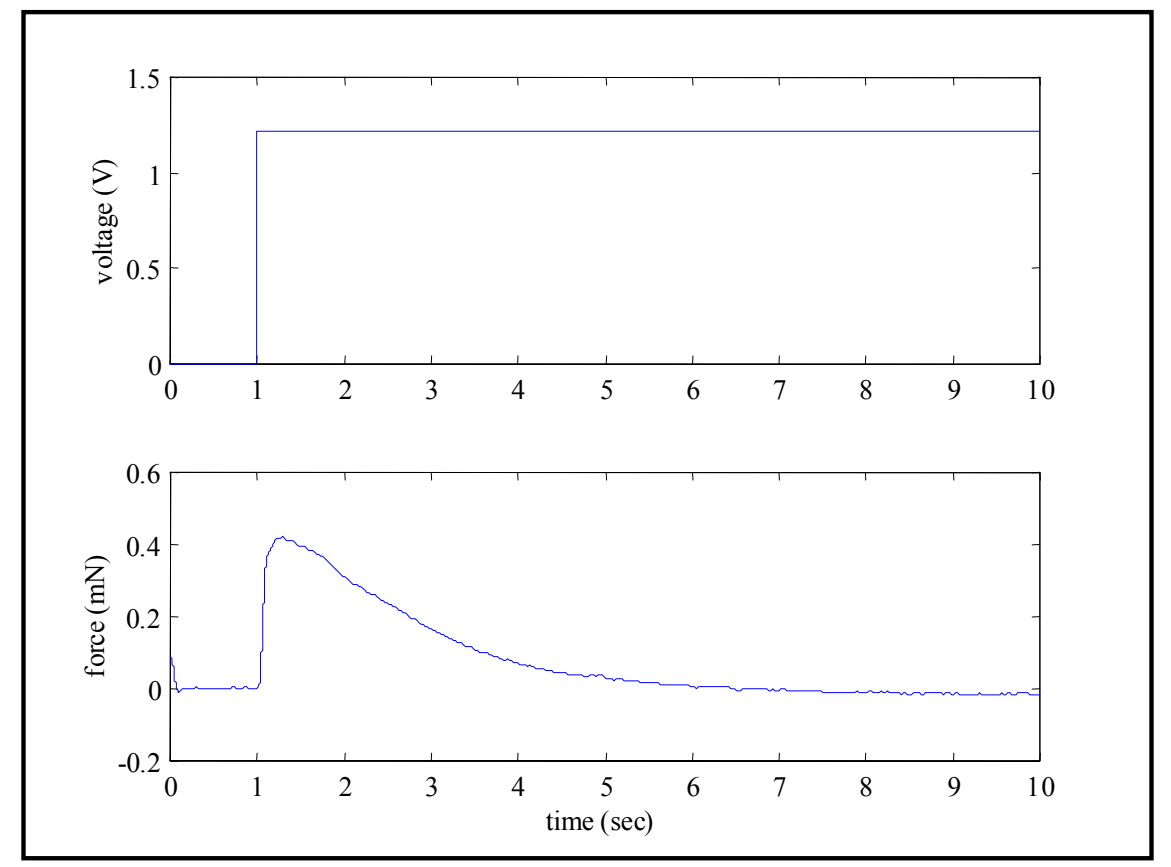

Figure 2.1: Blocked force response of an ionic polymer actuator to a $1.22 \mathrm{~V}$ step input.

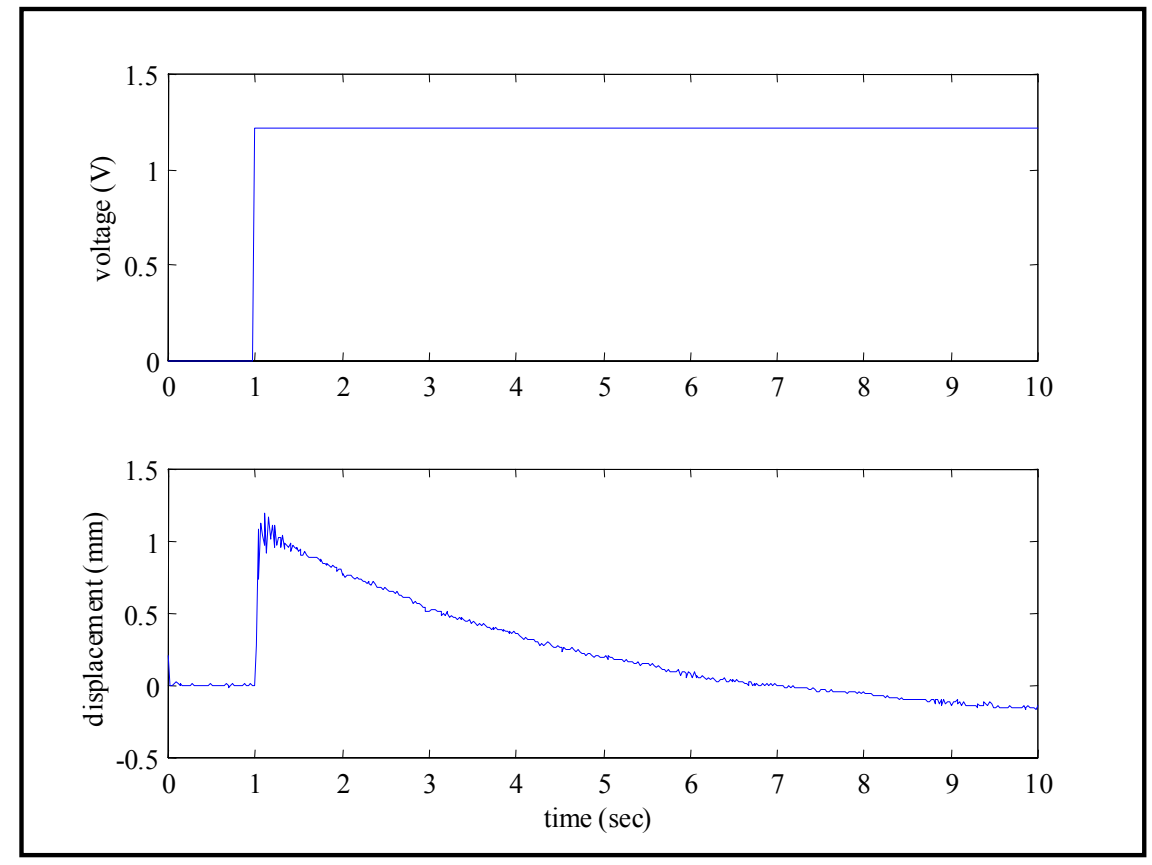

Figure 2.2: Free deflection response of an ionic polymer actuator to a $1.22 \mathrm{~V}$ step input. Note some resonance in the response when the step is applied. 


\subsection{Overview of Metrics}

In order to fully characterize the performance of the ionic polymer transducers used in this study, several different metrics were used. This section will discuss each of the metrics used in detail, explaining the significance of the metric, and the equipment and methods used to gather the metric.

\subsubsection{Surface Conductivity Measurements}

The purpose of this research is to develop new methods of plating the metallic electrode onto ionic polymer membranes for sensing and actuation applications. One of the key attributes of these electrodes is their electrical conductivity. As an illustration of this point, Shahinpoor and Kim showed that the force output of an ionic polymer actuator was increased by $50 \%$ when the conductivity of the electrodes was improved by electroplating additional metal over the existing electrode (53). In the current work the conductivity of the electrode was measured along the length (in-plane direction) of the membranes using a Fluke Model 87 digital multimeter set on the resistance mode. Two leads connected to this multimeter were placed one centimeter apart on the surface of the plated membrane and the indicated resistance recorded. These measurements were taken with the polymer fully hydrated with de-ionized water. No effort was made to remove water on the surface of the membrane prior to measurement of the resistance because the large resistivity of the water relative to that of the metal electrodes makes the contribution of the water to the resistance measurement negligible. Surface resistance measurements were taken at several points on both sides of each sample and averaged.

\subsubsection{Force-Deflection Measurements}

Ionic polymer membrane actuators operate by bending in response to an applied electrical input across their thickness. This bending could possibly be used to perform mechanical work. In order to measure the work capactiy of these devices, the amount of force and deflection generated by the bending motion should be measured. With conventional smart materials (e.g. piezoelectrics), the amount of force that is generated by the actuation of the material depends linearly on the deflection of the material from its neutral position. Therefore, if the deflection is identically zero, the amount of force that can be generated by 
the device is at its maximum. This is called the blocked force. Similarly, if the force that the device must overcome in order to move is identically zero, then the deflection of the device is at its maximum. This is called the free deflection. It has been shown by Newbury and Leo that ionic polymer membrane actuators exhibit a similar linear relationship between the induced force and deflection, although the forces and deflections reported herein for ionic polymer actuators are the maximum values generated over the actuation cycle, not the steady-state values $(37 ; 36)$. Because these devices have a characteristic linear forcedeflection curve, it is only necessary to measure the limiting cases (blocked force and free deflection) to build this curve. Once this data is obtained, the maximum work output of the devices may be determined and compared to other actuator technologies.

In order to measure the blocked force of ionic polymer membrane actuators, samples $5 \mathrm{~mm}$ wide by $17 \mathrm{~mm}$ long (free length) were mounted in a cantilever configuration between two gold foil electrodes. The samples were mounted such that the induced bending motion was normal to the force of gravity on the samples, thus eliminating any errors in the measurements due to gravitational effects - see Figure 2.3 for a photo of the setup used to measure the blocked force and free deflection. Once mounted, a load cell that had been previously zeroed was moved towards the tip of the sample using a lead screw until the load cell registered a non-zero force. Having made contact between the load cell and the ionic polymer membrane actuator in its neutral position, a voltage was applied to the membrane through the gold foil electrodes and the output of the load cell recorded. The load cell used to perform this test was a Transducer Techniques model GSO-10 and the output of this load cell was conditioned using a Transducer Techniques model TMO-1 signal conditioner. The applied step voltage was generated using a DSpace DS1102 controller board, and was amplified using a Hewlett-Packard model 6825A amplifier. The DSpace board was also used to collect the force data from the load cell, at a sampling rate of $1000 \mathrm{~Hz}$. This data was typically collected for 10-30 seconds, which was long enough to observe the fast peak and slow relaxation of the response. In order to facilitate the determination of the maximum force, the data was filtered to remove any noise using a fourth-order Butterworth filter with a cutoff frequency of $10 \mathrm{~Hz}$ in MatLab. The maximum load registered during the actuation of the sample is reported as the maximum blocked force of the particular device.

In order to measure the free deflection of the ionic polymer actuators, the same setup was used except that the load cell was backed away from the actuator using the lead 


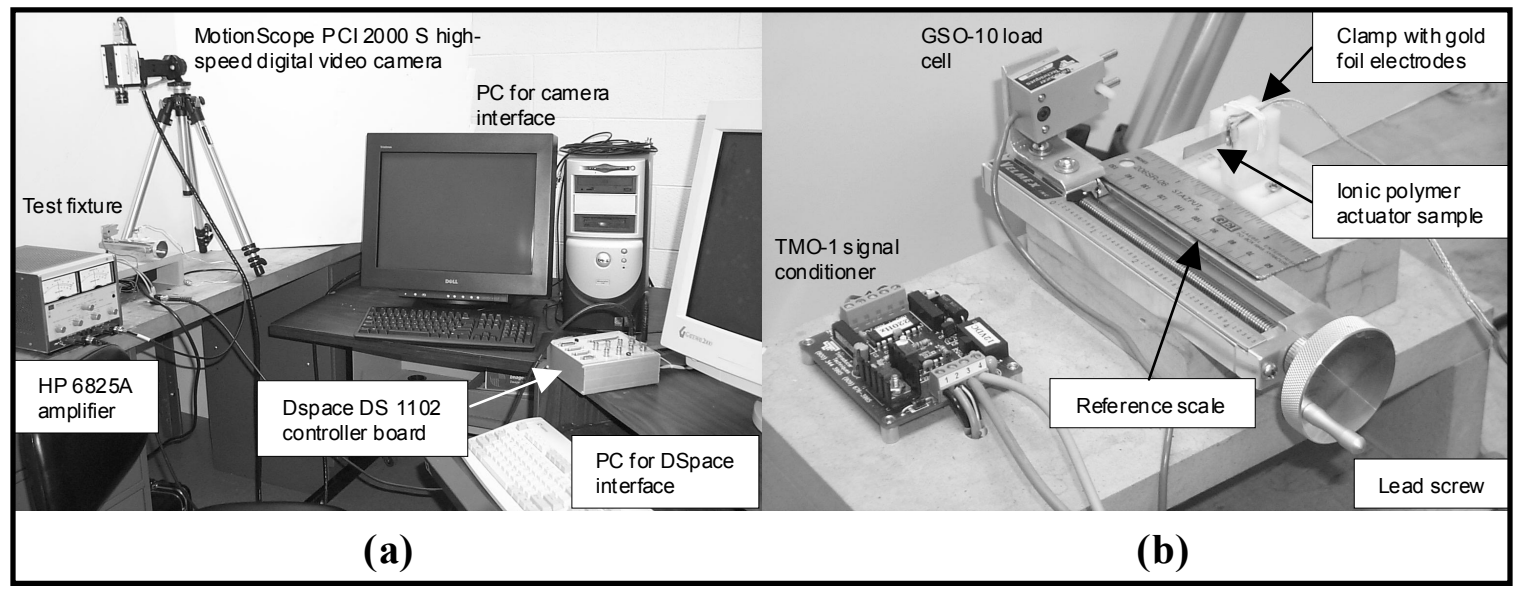

Figure 2.3: (a) Photo of the setup used to perform the force and deflection tests. (b) Detail of the test fixture.

screw. A high-speed digital video camera (MotionScope PCI 2000 S) was mounted to a tripod and positioned over the setup such that the tip of the ionic polymer actuator and a reference scale marked in millimeters were both in its field of view. A dot of White Out ${ }^{\mathrm{TM}}$ was applied to the tip of the actuator as a reference marker for the camera. As before, the DSpace DS1102 controller board was used to generate the step input to the polymer, which was amplified through a Hewlett-Packard 6825A amplifier. The polymer was allowed to deflect freely and its motion was recorded using the digital camera at a rate of 60 frames per second. The linear distance that the tip of the polymer deflected in response to the electrical input was then determined by using a motion tracking software package (Image Express v5.4A, Sensors Applications, Inc). The marker applied to the tip of the actuator and the reference scale placed in the field of view of the camera facilitated this tracking. It should be here noted that the reference scale was not in the same plane relative to the camera as the ionic polymer actuator, but was slightly below it (0.5-1.5 inches). Because this represents a small number relative to the distance from the camera to the setup, any errors introduced by this discrepancy were deemed negligible. Again, data were collected for 10-30 seconds. The maximum linear distance that the tip of the sample moved from its neutral position during this time is reported as the maximum free deflection of the particular device. 


\subsubsection{Sensing Measurements}

In addition to characterizing the Nafion ${ }^{\mathrm{TM}}$ membrane transducers as actuators, testing was also done to characterize them as sensors. Several researchers have shown that a transient voltage is produced across the faces of a metal-plated ionic polymer membrane when bent by an external force $(50 ; 19 ; 47 ; 34 ; 49 ; 35)$. More recently, Newbury and Leo have shown that the rate of charge flow across the membrane is related to the rate of the integrated strain $(37 ; 36)$. More generally, the short-circuit current through the membrane is proportional to the rate of the deformation of the membrane, effectively making it a direct velocity sensor. This important result may lead to new applications for these devices not previously considered. In order to quantify the sensitivity and linearity of these devices, a test fixture was developed by Mr. Kenn Newbury of the Center for Intelligent Material Systems and Structures at Virginia Tech. This fixture consists of a clamp for holding the polymer sample in a cantilevered configuration between two gold foil electrodes. The tip of the sample is then deflected by a high-displacement, low-force shaker (Acoustic Power Systems, Inc. model 113) - see Figure 2.4 for a schematic of the setup used to perform the sensing test. One of the electrodes on the membrane is held at ground and the other electrode is held at virtual ground through an ultraprecision operational amplifier (Analog Devices model OP177). Using the op-amp circuit, the short-circuit current through the membrane is measured - see Figure 2.4 for a diagram of this circuit. A random input is supplied to the shaker through an amplifier (MB Electronics model 2250MB Power Amplifier) and the actual displacement that the shaker imparts to the polymer is measured using a linear potentiometer (Novotechnik model T25). The random input is generated by a Tektronics model 2630 Fourier analyzer and the output of the op-amp circuit and potentiometer signal conditioner are input back into the Fourier analyzer. These signals were then used to create frequency responses between short circuit current and position from zero to $20 \mathrm{~Hz}$ using 512 spectral lines, averaged ten times. These frequency responses were saved to disk and post-processed using MatLab to convert them to frequency responses between short-circuit current and velocity by dividing each spectral band by its corresponding frequency and the imaginary unit, $i$. Because this frequency response is essentially linear in this frequency range, an average of the sensitivities over the 512 spectral bands from zero to $20 \mathrm{~Hz}$ may be taken and this number defined as the average sensitivity coefficient of the particular device 


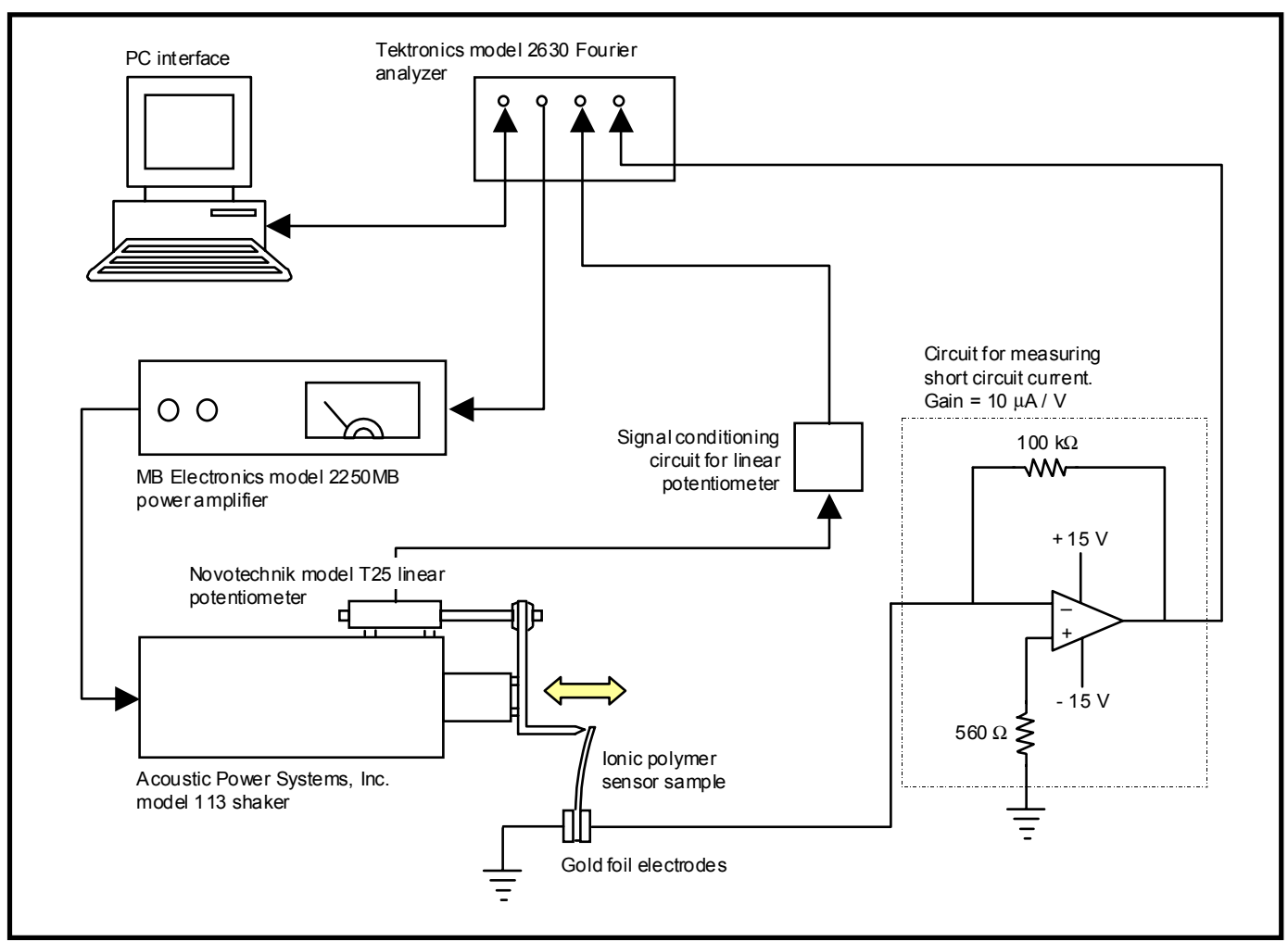

Figure 2.4: Schematic of the setup used to perform the sensing tests.

in that range. This allows for a quick comparison of the sensing capabilities of different ionic polymer membrane transducers, including different thickness transducers, different length transducers, and transducers made using different plating processes.

\subsubsection{Longevity Measurements}

One of the key issues identified with the use of non-precious metal electrodes during this research is the long-term stability of the electrodes, especially during actuation. In order to quantify this stability in terms of the actuator's effectiveness, a longevity test was developed. For this test, samples of plated Nafion ${ }^{\mathrm{TM}}$ membrane $10 \mathrm{~mm}$ wide by $35 \mathrm{~mm}$ long (free length) were clamped in a cantilevered configuration between two copper foil electrodes. The samples were then submerged in de-ionized water and a high-speed digital video camera (MotionScope PCI 2000 S) was positioned over the setup, such that the tip of each sample and a reference scale were both in the field of view of the camera. See Figure 2.5 for a photo of this setup. A dot of White Out ${ }^{\mathrm{TM}}$ was applied to the tip of the actuator as a marker. A sine voltage of $1.25-1.5 \mathrm{~V}$ (peak) at $1.0 \mathrm{~Hz}$ was then applied to each sample using a Hewlett- 


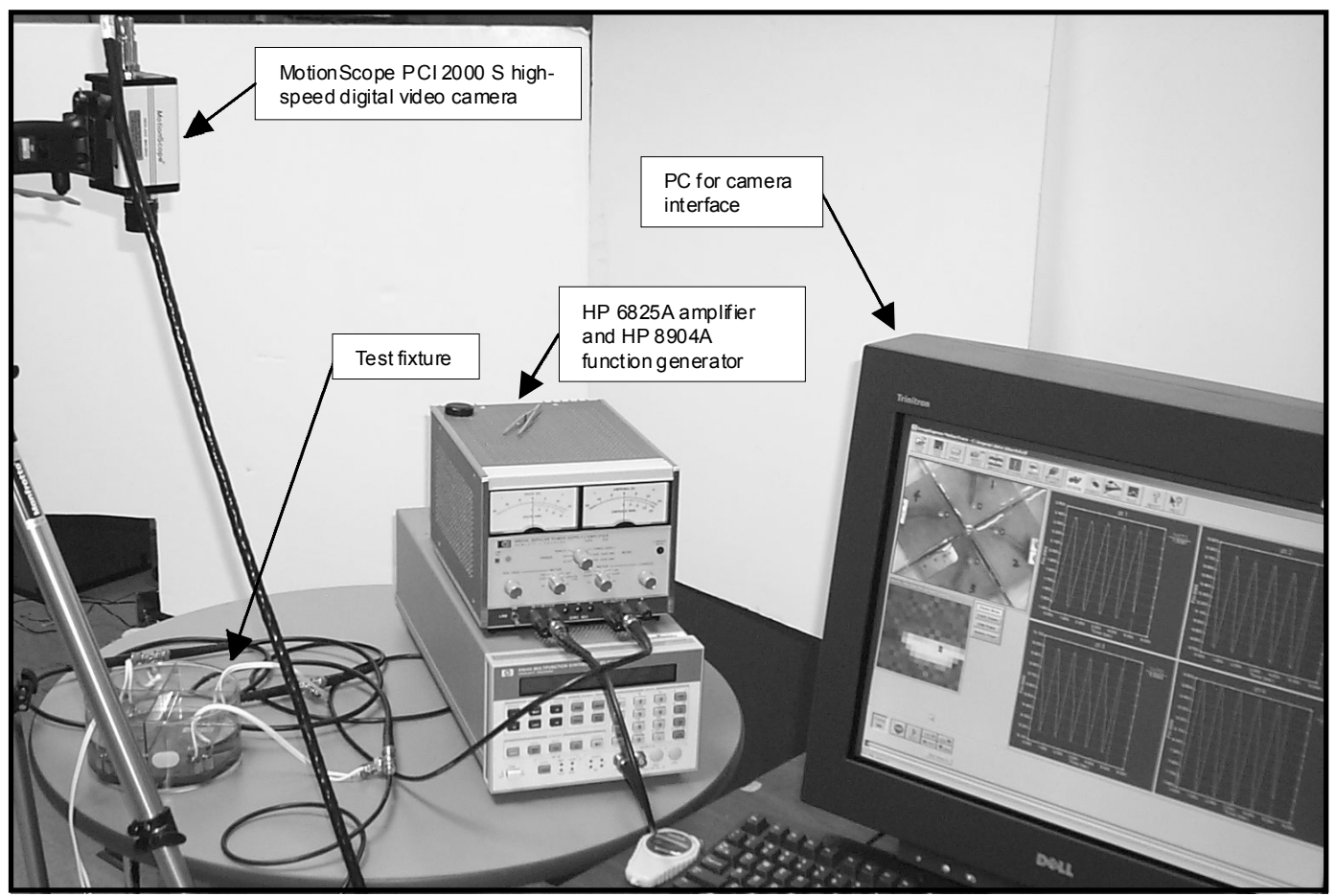

Figure 2.5: Photo of the setup used to perform the longevity testing.

Packard model 8904A function generator through a Hewlett-Packard 6825A amplifier. This signal was continuously applied to the samples for a period of time varying from two hours to several days. During this time the motion of the tip of each actuator was captured periodically using the high-speed video camera. The peak-to-peak free tip deflection of the samples was then obtained by post-processing the video data using a motion tracking software package (Image Express v5.4A, Sensors Applications, Inc). The linear tip deflection of the actuator was then plotted versus the number of actuation cycles, as determined by the frequency of the input signal and the elapsed time. This data was normalized by the initial value so that the longevity of different samples could be easily compared. If the electrodes were unstable, then the conductivity of the electrode would decrease when the sample was actuated. As the conductivity decreased, the tip deflection would also decrease. By measuring the amount that the tip deflection decreased, this test could quantify how well the actuator remained effective during actuation, and could give some insight into the stability of the metal electrodes. 


\subsubsection{Modulus Measurements}

Another interesting characteristic of these materials is their tensile elastic modulus, which will be useful in analytically determining strain energies and resonant frequencies and comparing these numbers to experimental results. In order to measure the elastic modulus, strips of plated Nafion ${ }^{\mathrm{TM}}$ were clamped on each end between two gold foil electrodes, one attached to mechanical ground and the other to a 100-gm load cell (Transducer Techniques, model GSO-100). The load cell was mounted through an angle bracket to a lead screw and a target was placed on the polymer clamp on the load cell side. See Figure 2.6 for a photo of the test fixture used to perform the modulus testing. A laser vibrometer (Polytec model OFV303) was positioned such that the path of the laser was parallel to the plane of the polymer and shone on the target on the movable clamp of the fixture. Both the output of the laser and the output of the load cell signal conditioner (Transducer Techniques model TMO-1) were recorded using a DSpace DS2002/2003 MUX AD board. The output of the load cell was also measured using a Hewlett-Packard model 34401A digital multimeter. A MatLab program written by Mr. Orion Parrot of Virginia Tech was used to collect data from the load cell and laser vibrometer controller (Polytec OFV3001). These data were collected as the tension in the polymer was cycled from zero to $100 \mathrm{gm}$ using the lead screw. After the load was run through three full cycles, the program post-processed the data using Hooke's Law relations to determine the modulus of the sample. The geometry (width and length) of the polymer was measured using a digital caliper (Mitutoyo model CD-6P) and entered into the program. The thickness used was the nominal thickness of the membrane (e.g. 180 microns, 125 microns, etc.). It should be here noted that one area of possible experimental error in this test is that the strain rate in the polymer was not controlled. Because Nafion ${ }^{\mathrm{TM}}$ is a viscoelastic polymer, its modulus is a function of the rate of the applied strain. In the described test, it was assumed that the rate of the strain was sufficiently small relative to the relaxation and creep effects in the polymer and that therefore the measured modulus will effectively be the steady-state modulus of the metal/Nafion ${ }^{\mathrm{TM}}$ composite.

\subsubsection{Density Measurements}

Another important characteristic of ionic polymer membrane transducers is their density. This is useful in determining the mass energy density of these devices and comparing them 


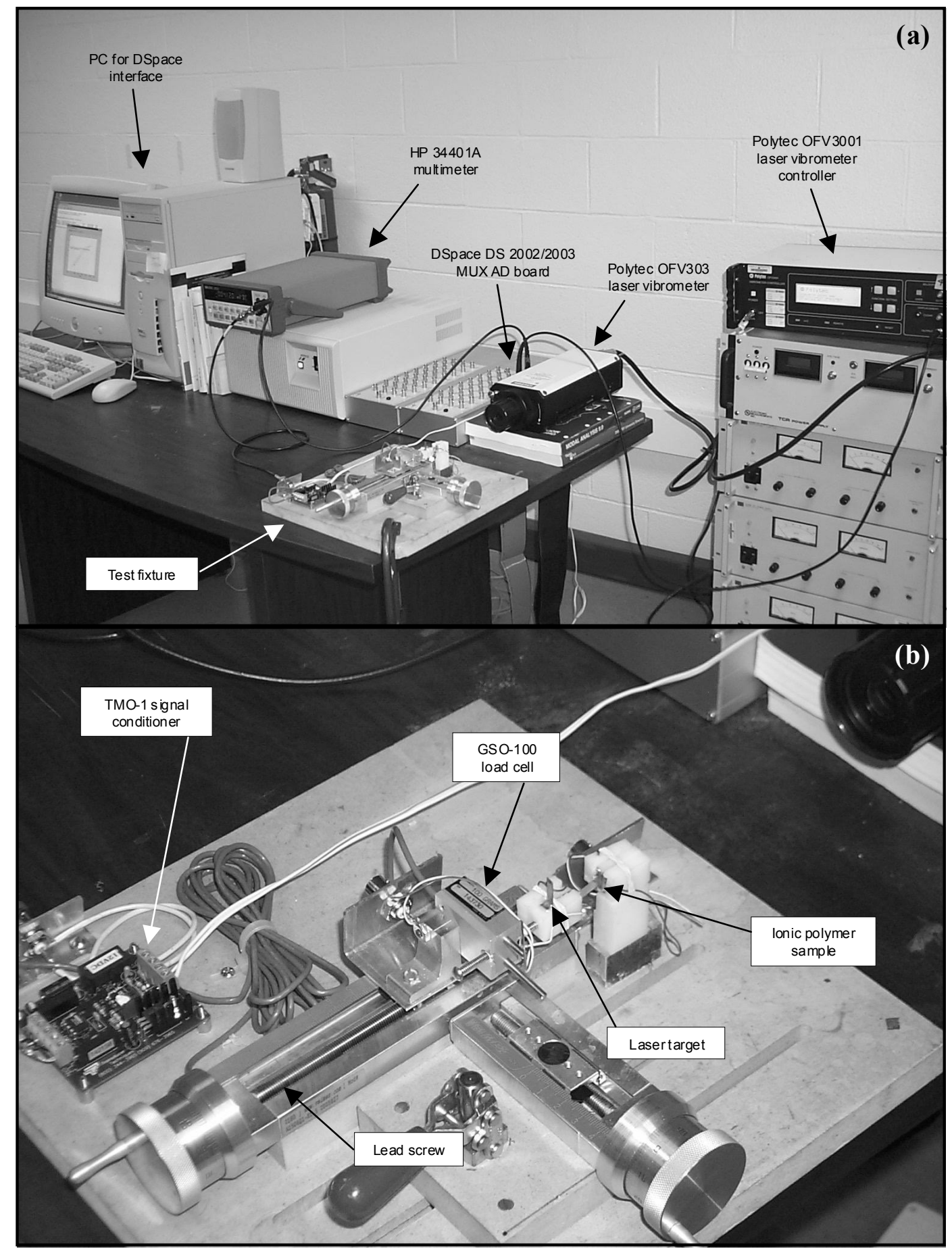

Figure 2.6: (a) Photo of the setup used to perform the modulus testing. (b) Detail of the test fixture. 
to other actuation and sensing technologies. In order to determine the density, the volume and mass of the samples must be known. The volume of the samples was calculated by multiplying their length, width, and thickness. The length and width of the samples was measured to the nearest half millimeter using a stainless steel scale. The thickness of the samples was taken as the nominal thickness of the membrane, provided by DuPont in the product literature (e.g. 180 microns, 125 microns, etc.). The mass of the samples was measured using an analytical balance (Mettler Toledo model AB204-S) with a resolution of $0.0001 \mathrm{gm}$. The samples were weighed in their fully hydrated state, but the water that clung to the surfaces of the samples was removed prior to weighing by shaking the samples vigorously. Typically, sheets of Nafion ${ }^{\mathrm{TM}}$ ranging in size from about 2 square inches to about 9 square inches were metal-plated. Smaller strips of this larger sheet were then cut and used for testing. In order to obtain an average density for a particular sheet, several samples of differing sizes were cut from the sheet and their densities measured. These densities were then averaged together to obtain an average mass density for the particular sheet. This average mass density was then used in all further calculations involving strips cut from that sheet.

\subsubsection{X-ray Photoelectron Spectroscopy}

In order to identify certain compounds present in the platings produced in this study, X-ray photoelectron spectroscopy (XPS) testing was performed. For this testing a Perkin-Elmer model 5400 XPS machine was used. The samples were prepared for the testing by drying under room air. Once dried, the samples were mounted on sample holders and transferred into the testing chamber, which was then evacuated to about $10^{-7}$ torr. A magnesium $\mathrm{K} \alpha \mathrm{X}$-ray source was used, which produces X-rays that impinge on the sample at 1253.6 $\mathrm{eV}$. The X-ray source was excited by electrons at $14 \mathrm{kV}$, and the input power was 300 $\mathrm{W}$. As the X-rays impinge on the sample, photoelectrons are released, which are detected and used to identify the elements in the sample. By measuring the binding energy of the released photoelectrons, the presence of componds in the sample may be detected. The photoelectrons are measured from a spot one millimeter by three millimeters on the sample surface and about 50 scans through the range of binding energies of interest are performed to reduce the effect of noise in the collected data. 


\subsubsection{Scanning Electron Microscopy}

High resolution images of the samples made in this study were captured using scanning electron microscopy (SEM) in order to quantify the thickness and composition of the electrodes and the degree of interlocking of the electrodes with the polymer membrane. Crosssectional micrographs of plated Nafion membranes were obtained by embedding the samples in epoxy and microtoming to obtain a smooth surface using a Reichert-Jung Ultracut E ultramicrotome with a diamond knife. This was done to get a good cross-section with minimal distortion. After microtoming, these samples were coated with approximately 5 nanometers of gold/palladium or platinum/palladium and images of the microtomed surface were captured using a Leo 1550 Field-Emmision Scanning Electron Microscope (FE-SEM) in the back-scatter mode with an accelerating voltage of 10 or $20 \mathrm{kV}$. The detector used for this imaging was a Robinson RBL1500 back-scatter detector. X-ray analysis was also performed in order to measure the composition of the electrodes. In this testing, the sample is bombarded with electrons and X-rays coming off of the sample are measured using an energy dispersive spectrometer (EDS) made by IXRF. This testing is different from the XPS testing in that individual elements can be identified, but not compounds. 


\section{Chapter 3}

\section{Description and Results of Initial Plating Methods}

\subsection{Introduction}

To date, ionic polymer transducers have seen use only in university laboratory settings as the subject of research. One of the reasons for the lack of commercial interest is the high cost of these devices, which is caused in part by the high cost of the precious metals that are typically used as the electrodes in ionic polymer devices. The goal of the current research is to reduce the cost of ionic polymer devices by reducing the cost of the metal electrodes. This chapter will discuss the initial work that was done in fabrication of ionic polymer transducers, including succesful demonstration of electromechanical transduction using electrodes made by sputter-coating gold, electroless plating of nickel, and impregnation/reduction plating of copper, including some secondary electroless gold plating. Each of these processes will be discussed and representative results demonstrating electromechanical transduction will be presented. The benefits and drawbacks of each process will also be addressed. Most importantly, the key issues associated with these processes and the use of non-precious metal electrodes in general will be identified.

Currently, the main research groups investigating ionic polymers as actuators and sensors are using either gold or platinum as the electrodes. It is the goal of this work to show that any conductive metal may be used as the electrode and to identify several key issues associated with the use of metals other than gold and platinum. 
Many of the current researchers working with ionic polymer membrane actuators use a process for the formation of the electrodes consisting of the following basic steps $(46 ; 1 ; 50 ; 33 ; 32 ; 29 ; 25 ; 52 ; 40)$

1. Mechanical roughening of the membrane by abrading with emory paper or sandblasting,

2. Hydrating the membrane in high temperature water,

3. Ultrasonic washing to remove surface particulates,

4. Washing in acid to remove surface oils and to saturate the membrane with protons,

5. Impregnating metal cations into the membrane by soaking in solution of the metal to be plated, and

6. Reducing the absorbed metal cations in aqueous bath.

Following this process the mobile cation may be exchanged for any other cation by soaking the plated membrane in a solution rich in that cation. Dr. Shahinpoor of the University of New Mexico has been making ionic polymer membrane actuators using variations of this process for years and reports the processing time to be around 30 hours (46). Because Nafion is a Teflon ${ }^{\mathrm{TM}_{-}}$-based polymer, adhesion of the membrane is an issue which is difficult to overcome. The process descibed above solves this problem by reducing the metal ions inside the membrane, thus producing an electrode which strongly interlocks with the polymer.

One of the goals of the current research is to investigate to what degree this interlocking is necessary for effective electromechanical transduction in ionic polymer membrane actuators. This will be done by investigating new methods for plating metals onto ionic polymer membranes that do not involve interpenetration of the metal into the polymer. The current work will also investigate other metals besides gold and platinum and their effectiveness as electrode materials in ionic polymer transducers.

\subsection{Description of Baseline Material}

In order to quantify the effectiveness of the new plating methods against a known material, a sample of plated Nafion ${ }^{\mathrm{TM}}$ membrane was obtiained from Dr. Shahinpoor of the University 
of New Mexico for use as a baseline material.

The baseline material kindly supplied by Dr. Shahinpoor consists of a Nafion ${ }^{\mathrm{TM}}-117$ membrane, of 180 micron nominal thickness, that has been plated on both surfaces with gold electrodes. Scanning electron micrographs of this plated membrane reveal that the two electrodoes display some morphological differences. One electrode consists of a diffuse layer of gold that extends more than 15 microns into the polymer while the other appears to terminate at a depth of 4 microns or so; both electrodes are further covered by a dense surface layer approximately 250 nanometers thick. Further imaging revealed that isolated gold particles exist throughout the polymer, extending even to the center of the membrane. Based on these images it is apparent that the electrodes were deposited by making use of the ion exchange properties of the Nafion ${ }^{\mathrm{TM}}$ polymer, perhaps by using a diffusion process such as the one employed by Takenaka and Torikai $(58 ; 59)$.

Much testing on samples of this baseline material has been performed in the Center for Intelligent Material Systems and Structures here at Virginia Tech, including measurements of the useful energy density, sensing capability, and modulus of elasticity. This testing has revealed that this material exhibits a large bending strain when actuated by a low voltage and produces a voltage across its electrodes when suddenly bent. The material exhibits no noticeable degredation and samples have been stored and tested for several months with no loss of actuation performance or loss of adhesion of the gold electrodes.

As mentioned, the free cation in Nafion ${ }^{\mathrm{TM}}$ can be easily exchanged for another cation, which will affect the performance of the material. According to Dr. Shahinpoor, the mobile cation in the sample of baseline material is lithium. Research by Shahinpoor and Kim (52) and by Abe et al. (1) has confirmed that the lithium ion results in larger generated actuation forces and displacements than the other uni- and di-valent cations studied.

\subsection{Gold Sputter-Coating}

The initial approach of the current research was to determine to what extent the interlocking of the metal electrode with the polymer membrane was necessary. As a first step, gold electrodes were sputter-coated onto dry Nafion membranes. Recently, Zhou et al. have used a sputtering process with subsequent electroplating to plate gold onto Nafion ${ }^{\mathrm{TM}}$ membranes (67). In their work, the membranes were initially roughened by 1500 grit sandpaper 
and then cleaned with hydrochloric acid, they were then dried under nitrogen. After drying, chromium, platinum, silver, or gold was sputtered onto the Nafion ${ }^{\mathrm{TM}}$ membrane to a thickness of about 0.4 microns, followed by gold electroplating to a thickness of about 2.2 microns. These researchers found that unless the initially sputtered metal layer was gold, cracks would form between the sputtered layer and the electroplated layer when high voltages $(20 \mathrm{~V})$ were applied, causing delamination of the electroplated gold layer.

The current investigation into new coating methods was begun with sputter-coating because it is relatively common, fast, and controllable. The sputtering process used in this study is typically employed to coat a layer of a noble metal onto a substrate prior to SEM testing, and is capable of depositing very thin coatings.

\subsubsection{Approach}

In order to sputter-coat gold electrodes onto the Nafion ${ }^{\mathrm{TM}}$ membranes, some pretreatment of the membrane is necessary. The membrane is first roughened by sandblasting with fine glass beads and is then cleaned using an ultrasonic cleaner. Following this treatment the membrane is rinsed under de-ionized water and dried at room temperature for several hours. This goal of this roughening and cleaning treatment is to improve the adhesion of the sputtered gold to the Nafion ${ }^{\mathrm{TM}}$ membrane.

Following the pretreatment, sputter coating was performed using a Bal-Tec SCD005 sputter coater. The sputtering is carried out under an atmosphere of inert argon gas. In order to remove any contaminants from the sputtering chamber, the chamber is initially evacuated to a pressure of 0.02 millibar, with occasional flushing of argon gas. The chamber is then back-filled with argon gas to a pressure of 0.05 millibar and the sputtering is initiated. Sputter-coating is performed using a current of $30 \mathrm{~mA}$ for 3 minute intervals. The sputtering is limited to 3 minutes in order to prevent overheating of the membrane or the sputter-coater. In order to deposit thicker gold coatings, the sputtering process is repeated. See Figure 3.1 for a photo of samples of Nafion ${ }^{\mathrm{TM}}$ that had been coated for one, two, three, and four plating cycles.

\subsubsection{Results}

Results with this method showed that the electromechanical response of the actuators was evident, but significantly diminished as compared to gold-plated actuators obtained from 


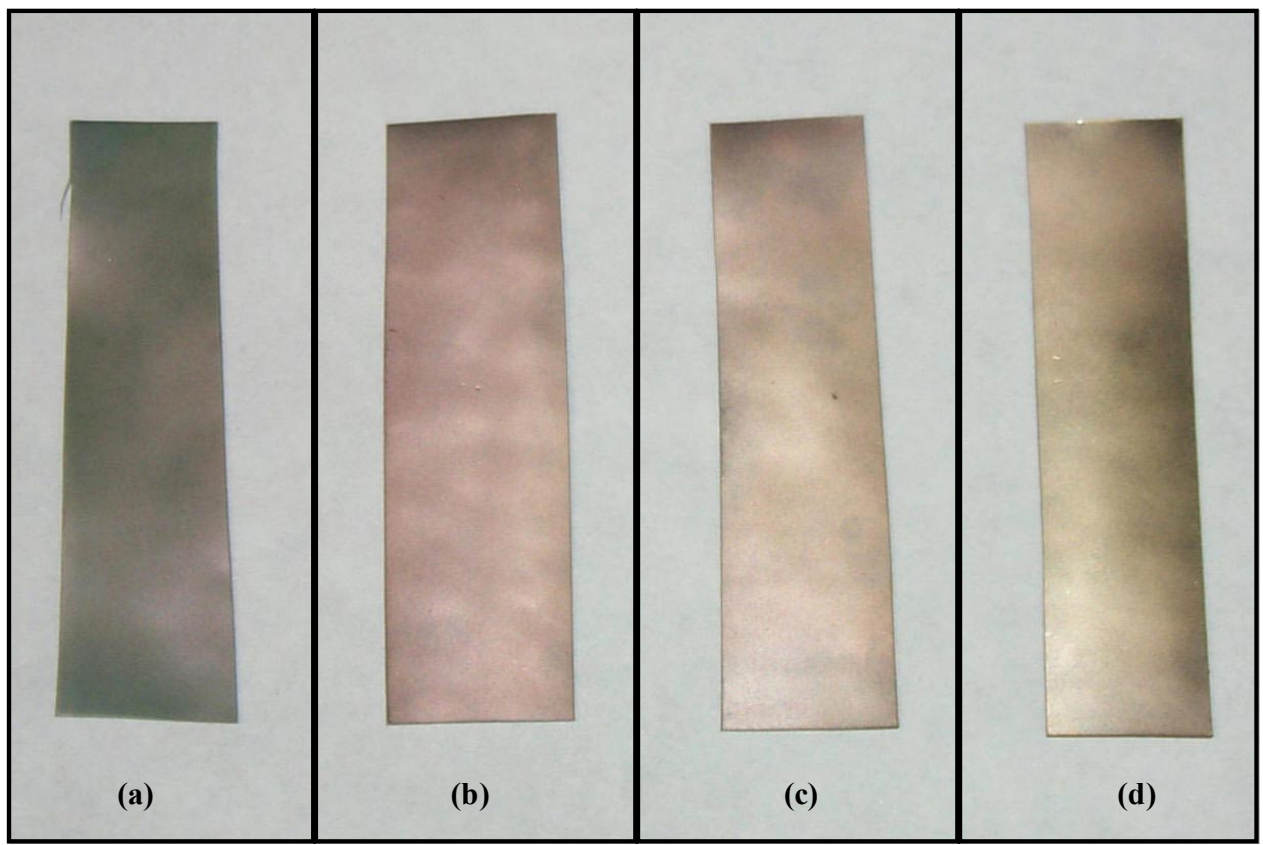

Figure 3.1: Photos of samples of Nafion ${ }^{\mathrm{TM}}$ membrane sputtered with gold for (a) one, (b) two, (c) three, and (d) four coating cycles.

Dr. Shahinpoor, which were used as a baseline. One reason for the drop in performance is the high surface resistance of the sputtered electrodes. The sputtering process was repeated up to four times in order to improve the surface resistance of the gold electrodes - see Figure 3.2 for a plot of the measured surface resistance versus the number of sputtering cycles. Please note that one sputtering cycle represents gold sputter-coating for 3 minutes per side, and the surface resistances were measured as described in Chapter 2. As can be seen from the figure, the surface resistance of the gold electrodes decreases dramatically as more gold is applied to the membrane, but appears to reach a minimum value of about $7.5 \Omega / \mathrm{cm}$. It is possible that this is a fundamental limitation of the sputtering process. Figure 3.3 shows scanning electron micrographs of the surfaces of gold sputtered Nafion ${ }^{\mathrm{TM}}$ membranes for one, two, three, and four sputtering cycles. From this figure it is evident that although the average grain size of the gold increases as the gold coating grows thicker, even for a sample that has been coated four times the relative percentage of the electrode area that is occupied by microcracks and grain boundaries is rather large, owing to the high measured surface resistance of these coatings.

Another problem with the gold sputtering process is the robustness of the electrodes made thereby. The membrane must be in a dry state during the sputtering process and 


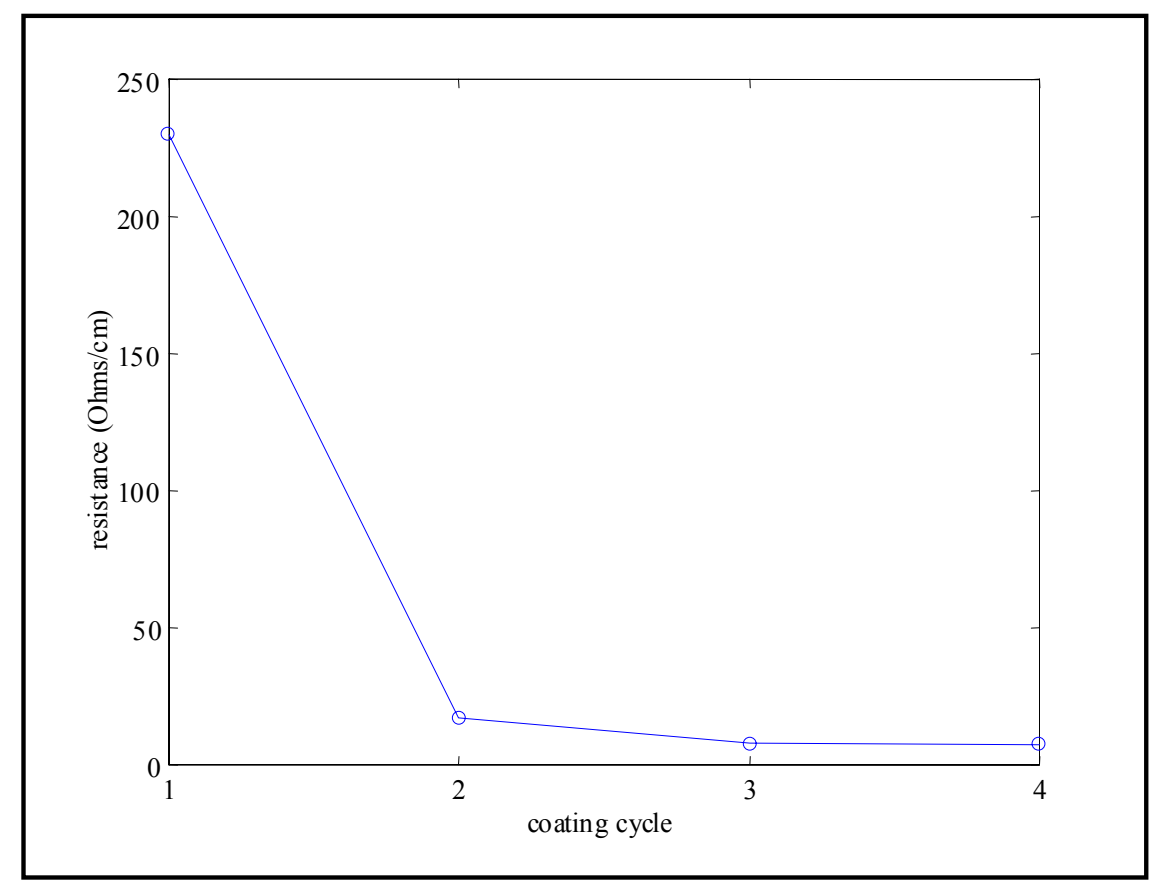

Figure 3.2: Measured surface resistance of the sputtered gold coating as a function of coating cycle.

then hydrated after. Because of the large amount of water that the Nafion absorbs during hydration, it swells in size and stretches the gold electrode, increasing its resistance. As-plated, the surface resistance was measured to be approximatedly $7.5 \Omega / \mathrm{cm}$; once hydrated, this number may become as high as $100 \Omega / \mathrm{cm}$. Bar-Cohen has reported a similar phenomenon in a gold-plated Flemion ${ }^{\mathrm{TM}}$ sample (a carboxylic acid membrane, product of Asahi glass company, Japan) (3). The effect of hydration and swelling of the membrane on the conductivity of his electrodes was less pronounced, however. This is likely because the electrodes used in his study were deposited by an impregnation/reduction process similar to the one described in Section 3.1.

Also problematic was the adhesion of the gold electrodes to the Nafion ${ }^{\mathrm{TM}}$ membrane. Although the gold seemed well-adhered to the membrane following the sputtering, once hydrated the gold could be removed from the membrane simply by rubbing with one's finger or gripping the membrane with a clamp. This loss of adhesion is likely due to the extreme difficulty in bonding to Teflon ${ }^{\mathrm{TM}}$ substrates, on which Nafion ${ }^{\mathrm{TM}}$ is based. The problem is compounded by the fact that the membrane swells so much during hydration, which is likely to generate streses between the Nafion ${ }^{\mathrm{TM}}$ membrane and the gold electrodes.

Regardless of the problems associated with the gold sputter-coating process, electro- 


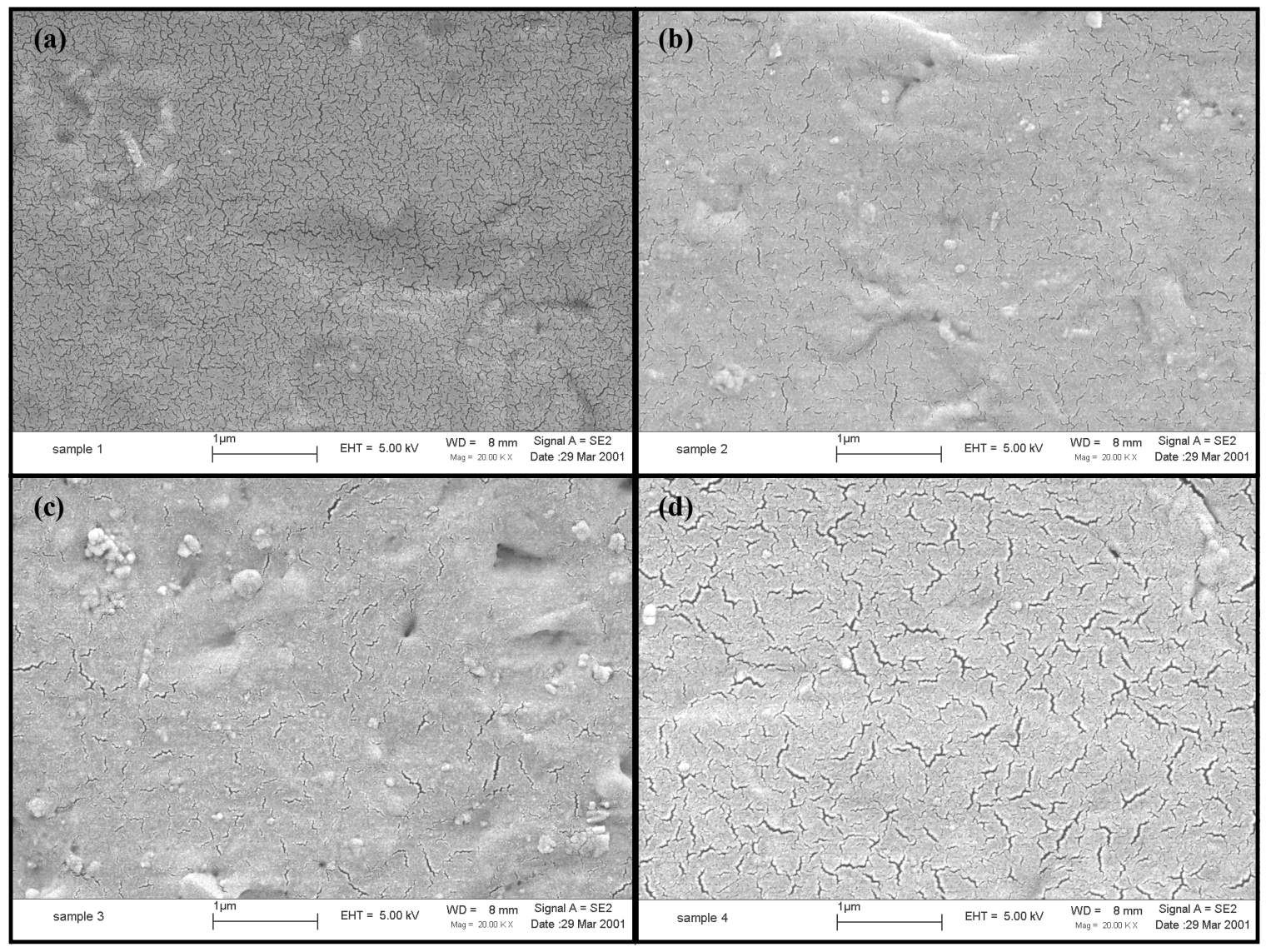

Figure 3.3: Scanning electron micrographs of the surface of samples of Nafion ${ }^{\mathrm{TM}}$ that were sputter-coated with gold for (a) one sputtering cycle, (b) two sputtering cycles, (c) three sputtering cycles, and (c) four sputtering cycles.

mechanical transduction in the samples made using this process was evident. Please refer to Figure 3.4 for a plot of the free tip displacement of a strip of gold-plated Nafion ${ }^{\mathrm{TM}}$ to a $1.5 \mathrm{~V}$ square wave input; the sample is $5 \mathrm{~mm}$ wide with a $20 \mathrm{~mm}$ free length. This sample had been subjected to two sputtering cycles. As can be seen from the figure, the tip of this strip moved on the order of 250 microns in response to the actuation voltage. Figure 3.5 shows a plot of the measured blocked force of a sample that was sputter-coated with gold for one sputtering cycle by the process described above. Again, the sample was $5 \mathrm{~mm}$ wide with a $20 \mathrm{~mm}$ free length. Both of these samples were treated such that the free cation was sodium by soaking in concentrated sodium chloride solution overnight. From Figure 3.5, it is evident that this sample generated over $0.5 \mathrm{mN}$ of force for a $4.0 \mathrm{~V}$ input. By contrast, strips of the baseline material from UNM the same size have been found to generate tip displacements on the order of 500 microns and blocked forces on the order of $0.8 \mathrm{mN}$ for 


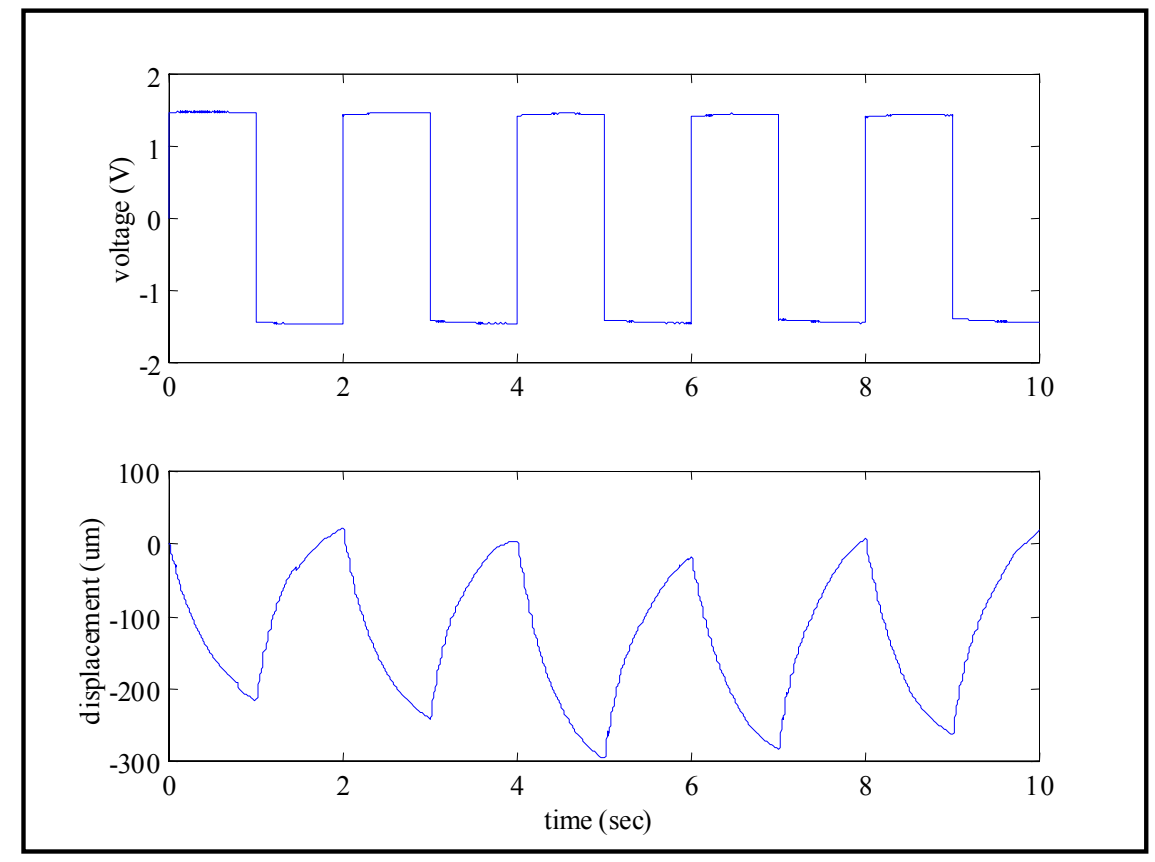

Figure 3.4: Free tip deflection versus time of a sample sputtered with gold for two sputtering cycles. The sample was $5 \mathrm{~mm}$ wide with a $20 \mathrm{~mm}$ free length and the input was a $1.5 \mathrm{~V}$ square wave.

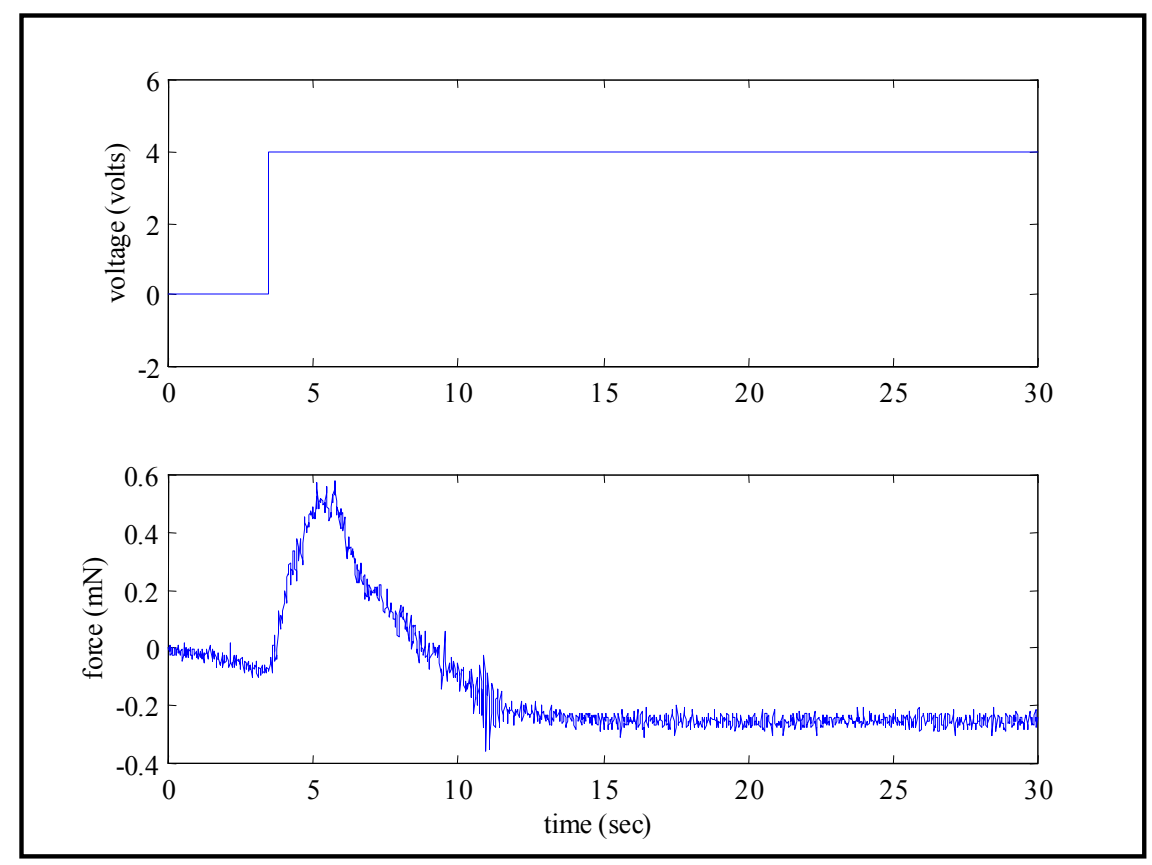

Figure 3.5: Blocked force response to a $4.0 \mathrm{~V}$ step input of a strip of Nafion ${ }^{\mathrm{TM}}$ that had been coated with gold for one sputtering cycle. This sample was $5 \mathrm{~mm}$ wide with a $20 \mathrm{~mm}$ free length. 
1.0 $\mathrm{V}$ inputs and greater than 700 microns of free tip displacement and more than $1.5 \mathrm{mN}$ of blocked force for $1.25 \mathrm{~V}$ inputs $(37 ; 36)$. From these results it is clear the sputter-coated gold samples are generating forces and displacements that are on or near the same order of magnitude as the baseline material, but at much higher input levels, indicating that the electromechanical coupling in the gold sputtered material is lower than in the baseline material.

Because the sputter-coating process results in only a surface deposition, the interlocking associated with the impregnation/reduction process is non-existent. These results suggest that interlocking of the metallic electrode with the ionic polymer membrane is not

necessary for electromechanical transduction to take place, but that the interlocking may be desireable for good adhesion and stability of the electrode. Further, it is also apparent that the plating process should be performed in aqueous solution and with the membrane fully hydrated in order to acheive higher conductivity in the electrodes and improved adhesion of the electrode to the membrane.

\subsection{Electroless Nickel Plating}

Although the gold sputtering process did produce functional actuators, an aqueous plating process seemed more suitable to plating Nafion ${ }^{\mathrm{TM}}$ membranes. After some initial experimentation, an electroless nickel plating process was chosen. Electroless nickel deposition was first developed by A. Brenner and G. Riddell in 1946. These coatings are used in the aerospace, automotive, computer, and oil and gas industries as decorative, protective, and conductive finishes. The main benefit of the process is the excellent thickness uniformity, even in blind holes and recesses (6). A well-known electroless nickel process is developed by reducing nickel chloride using sodium hypophosphite at elevated temperature. This plating proceeds according to the following chemical reaction:

$$
\mathrm{Ni}^{2+}+\mathrm{H}_{2} \mathrm{PO}_{2}+\mathrm{H}_{2} \mathrm{O}--->\mathrm{Ni}^{0}+\mathrm{H}_{2} \mathrm{PO}_{3}+2 \mathrm{H}^{+}
$$

\subsubsection{Approach}

The electroless nickel plating was performed according to the reaction above in aqueous solution at elevated temperature. The plating chemicals were supplied by Buehler as product number 20-8192 Edgemet ${ }^{\mathrm{TM}}$ Kit. In order for this reaction to take place, a catalyst 
must be present. In some cases the catalyst may be the substrate that is being plated, but for plastic substrates, a catalyst must first be plated onto the surface. A common technique for plating nickel or copper onto Teflon ${ }^{\mathrm{TM}}$ printed circuit boards is to first plate a thin layer of palladium as a catalyst. There are a number of companies selling systems to accomplish this; the one used in this work was supplied by Solution Technology Systems, of Redlands, CA as their DIRECT ${ }^{\mathrm{TM}}$ technology. This palladium plating system consists of four solutions, HN502 $\mathrm{S}^{\mathrm{TM}}$ conditioner, HN503 $3^{\mathrm{TM}}$ pre-dip, HN504 ${ }^{\mathrm{TM}}$ activator, and $\mathrm{HN} 505^{\mathrm{TM}}$ accelerator. Prior to any plating treatment, the membrane is sandblasted on both sides, washed in an ultrasonic cleaner, and hydrated by boiling in de-ionized water for one hour. The first step in the palladium deposition process is the HN502S ${ }^{\mathrm{TM}}$ conditioner, which is an alkaline solution designed to remove oil, oxidation, and soil from the substrate. The membrane is soaked in the conditioner at $80{ }^{\circ} \mathrm{C}$ for three minutes, with agitation by a magnetic stirrer. The membrane is then rinsed under hot running tap water for one minute, and soaked in the HN503 ${ }^{\mathrm{TM}}$ pre-dip at room temperature for 20 seconds, with agitation by shaking. The purpose of the pre-dip is to displace the rinse water from the surface of the membrane and prevent excess water from being carried into the activator solution. After the pre-dip, the membrane is placed directly into the HN504 ${ }^{\mathrm{TM}}$ activator solution for three minutes at $41{ }^{\circ} \mathrm{C}$, with agitation by a magnetic stirrer. The activator solution is identical to the pre-dip, with the addition of a tin/palladium colloidal catalyst. After the activator, the membrane is again rinsed under hot running tap water and then placed into the $\mathrm{HN} 505^{\mathrm{TM}}$ accelerator solution at $60{ }^{\circ} \mathrm{C}$ for 3 minutes. The accelerator serves to merge the colloids deposited by the activator into a continuous film and increase the plating potential of the treated parts. This process was derived from a process to plate through-holes on Teflon ${ }^{\mathrm{TM}}$ printed circuit boards. Once the palladium layer is deposited, nickel is plated over the palladium using the solutions supplied by Bueller. These solutions, Edgemet ${ }^{\mathrm{TM}}$ solution A, which contains 5-10\% nickel chloride, and Edgemet ${ }^{\mathrm{TM}}$ solution B, which contains 1-5\% sodium hypophosphite, are combined in equal volumes and heated to $78{ }^{\circ} \mathrm{C}$ on a hot plate. The palladium-treated membrane is added to the heated and mixed solutions and plating is allowed to take place for 5-20 minutes, with agitation by a magnetic stirrer. This temperature was found by experimentation to give good quality nickel coatings; the thickness of the plating could be changed by adjusting the plating time. Because the nickel ions will only reduce at the palladium interface, this process results in a purely surface deposition of 
metal, like the gold sputter-coating process.

\subsubsection{Results}

Results with this process showed that the electromechanical response of the actuators was evident, but the performance was not as good as the baseline material. The Nafion ${ }^{\mathrm{TM}}$ membranes treated with this process were about 3-8 in ${ }^{2}$ in size. Strips of nickel-plated Nafion $^{\mathrm{TM}} 5 \mathrm{~mm}$ wide and about $30 \mathrm{~mm}$ long were cut from these larger pieces and clamped in a cantilevered configuration with a $20 \mathrm{~mm}$ free length. The blocked force and free deflection at the tip of these strips was measured for voltage inputs of 1.0-5.0 V, as described in Chapter 2. Results with this process were very scattered, with little consistency between responses of membrane samples plated on different days, strips cut from the same plated sample, or even the same strip actuated repeatedly. Although plated strips were tested that produced no measurable force in repsonse to an actuation voltage, some samples were found to produce as much as $6 \mathrm{mN}$ of force. Although there was a great deal of scatter in the measured data, the nickel-plated samples were found to produce force on the order of about $0.18 \mathrm{mN}$ per volt at voltage levels of 2.0-3.0 V. Very little force was measured at lower voltage inputs. By comparison, strips of the baseline material the same size obtained from UNM have been measured to produce blocked force at a level of about $0.8 \mathrm{mN}$ per volt at voltage levels of $0.75-1.0 \mathrm{~V}$, more than four times that produced by the nickel-plated samples $(37 ; 36)$. See Figure 3.6 for a plot of the measured blocked force versus time for a nickel-plated sample and a sample of the baseline material. These results indicate that as with the gold sputter-coated samples, the electromechanical coupling is lower in the nickel-plated Nafion ${ }^{\mathrm{TM}}$ actuators than in the baseline material.

From this figure it is apparent that the baseline material produces more force than the nickel-plated sample, and at a lower actuation voltage. Another important difference between these two responses is evident, however. The baseline material has a much faster time constant than the nickel-plated material. Whereas the sample from UNM reaches its peak force of about $1.4 \mathrm{mN}$ in about 0.15 seconds, the nickel-plated sample does not reach its peak force until almost 2 seconds after the step voltage is applied. Both the magnitude of the peak force produced by the nickel-plated samples and the rise time of the blocked force response was found to vary across the range of samples tested. Figure 3.7 shows a

plot of the blocked force response of a nickel-plated strip that produced almost $6 \mathrm{mN}$ of 
measured blocked force for a $3.0 \mathrm{~V}$ step input. This sample has a very slow characteristic rise time; from the figure it can be seen that the peak force did not occur until 15 seconds after the step was applied.

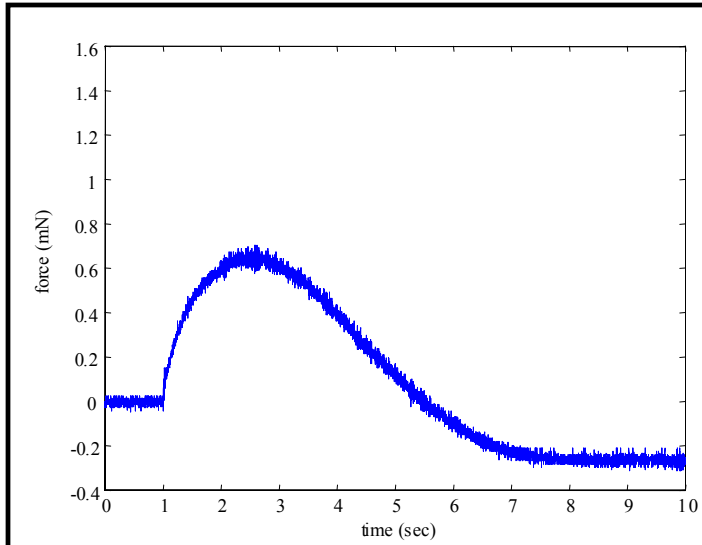

(a)

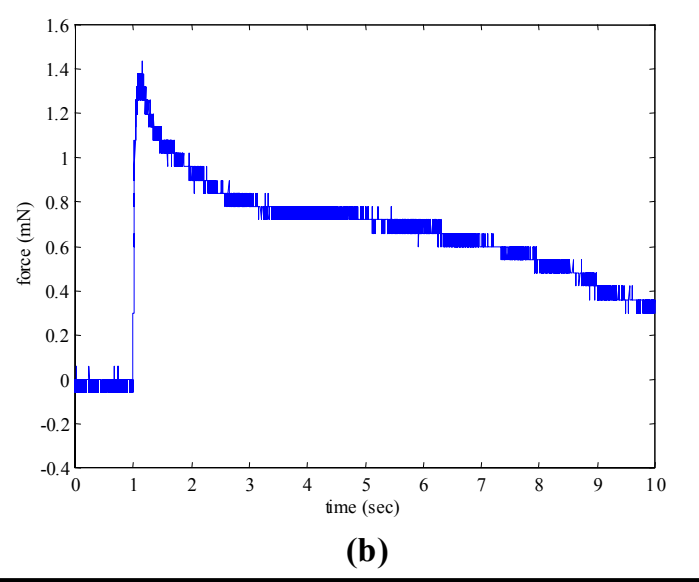

(b)

Figure 3.6: (a) Blocked force response of a nickel-plated Nafion ${ }^{\mathrm{TM}}$ membrane to a 3.0 V input, (b) Blocked force response of the baseline material to a $1.5 \mathrm{~V}$ input. Both samples were $5 \mathrm{~mm}$ wide with a $20 \mathrm{~mm}$ free length.

Also, significant adhesion problems were encounted when using this process. Although electrodes with relatively low surface resistance measurements were obtained (2-3 $\Omega / \mathrm{cm}$ ), the electrodes would typically crack and flake off upon application of the electric field. The poor performance of the actuators prepared in this way was initially attributed to this loss of adhesion of the electrode. The poor adhesion was attributed to the high compliance and Teflon ${ }^{\mathrm{TM}}-$ like properties of the Nafion ${ }^{\mathrm{TM}}$ membrane.

One way to improve adhesion is through the use of an etching process, like plasma etching. Takenaka et al. have used gas plasma etching to improve the adhesion of catalyst layers to Nafion ${ }^{\mathrm{TM}}$ membranes (59). This etching can also be performed by a chemical process. A well-known chemical etching process for Teflon ${ }^{\mathrm{TM}}$ is called Tetra-Etch ${ }^{\mathrm{TM}}$ and is made by W L Gore and Associates. Tetra-Etch ${ }^{\mathrm{TM}}$ consists of a sodium-napthalene complex in glycol ether solution. It works by stripping a layer of fluorine atoms off of the surface of the polymer, allowing functional groups to form when the surface is exposed to water and air. This increases the surface energy of the Teflon ${ }^{\mathrm{TM}}$ and can significantly improve the adhesion of coatings. Like the palladium deposition process discussed above, chemical etching is used extensively in the plating of Teflon ${ }^{\mathrm{TM}}$ printed circuit boards. Also, BarCohen et al. have used Tetra-Etch ${ }^{\mathrm{TM}}$ to treat metal-plated Nafion ${ }^{\mathrm{TM}}$ membranes prior to 


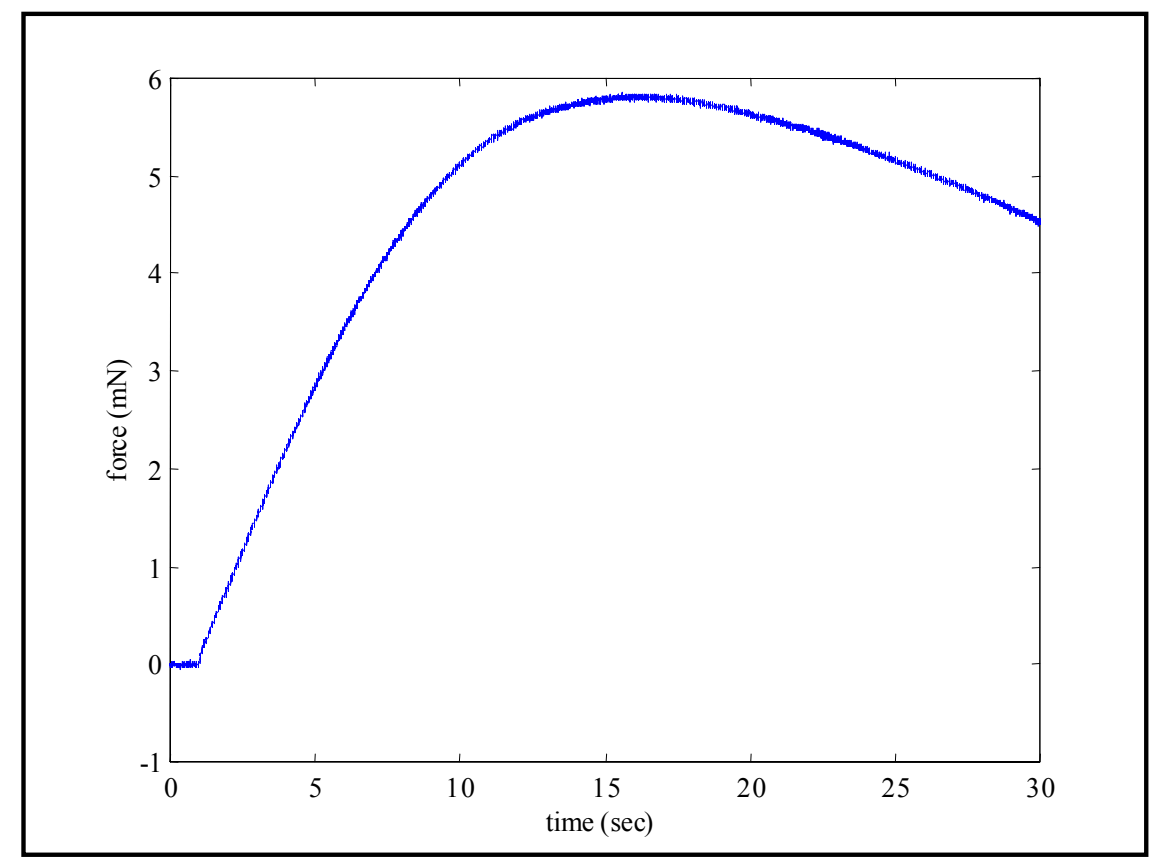

Figure 3.7: Blocked force response of a nickel-plated sample to a $3.0 \mathrm{~V}$ step input. The sample was $5 \mathrm{~mm}$ wide with a $20 \mathrm{~mm}$ free length.

the application of a barrier coating, which was meant to prevent the dehydration of the material (5). They found that without using the etching treatment, the barrier coating was easily peeled off. When the membranes were etched, the coating showed up to four times improvement in the adhesion, as measured by a peel test. Tetra-Etch ${ }^{\mathrm{TM}}$ was used to a limited extent in the current study, but because it did not seem to improve adhesion of the nickel to the membrane, and because it is a very dangerous substance and requires elaborate safe handling procedures, such chemical etching was quickly abandoned.

Other attempts were made to improve the adhesion of the nickel to the Nafion ${ }^{\mathrm{TM}}$ membrane. The first such attempt involved the design and construction of a fixture to tension the membrane and hold it flat in the plating solutions. See Figure 3.8 for a photo of this fixture. Prior to this development, the membranes were not well fixtured during the plating process and as a result they became creased and wrinkled - see Figure 3.9 for a photo of a sample that was nickel-plated without using this fixture. While this fixture did improve the quality of the nickel plating, it suffered from several drawbacks. First, it only provided tension in one direction, which limited its effectiveness in holding the membrane flat. Second, it allowed only for very small samples to be made. Another approach was to change the roughening treatment of the membrane prior to the plating process. The 


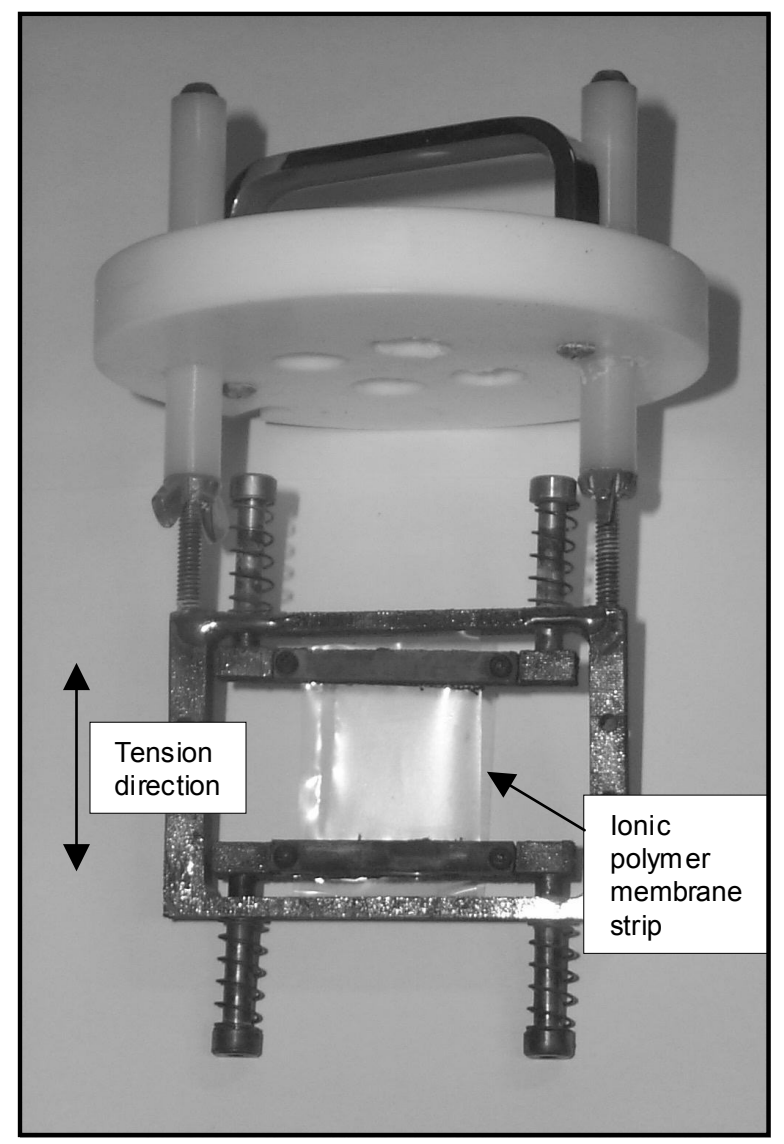

Figure 3.8: Photo of the tension fixture, with a membrane sample mounted in place.

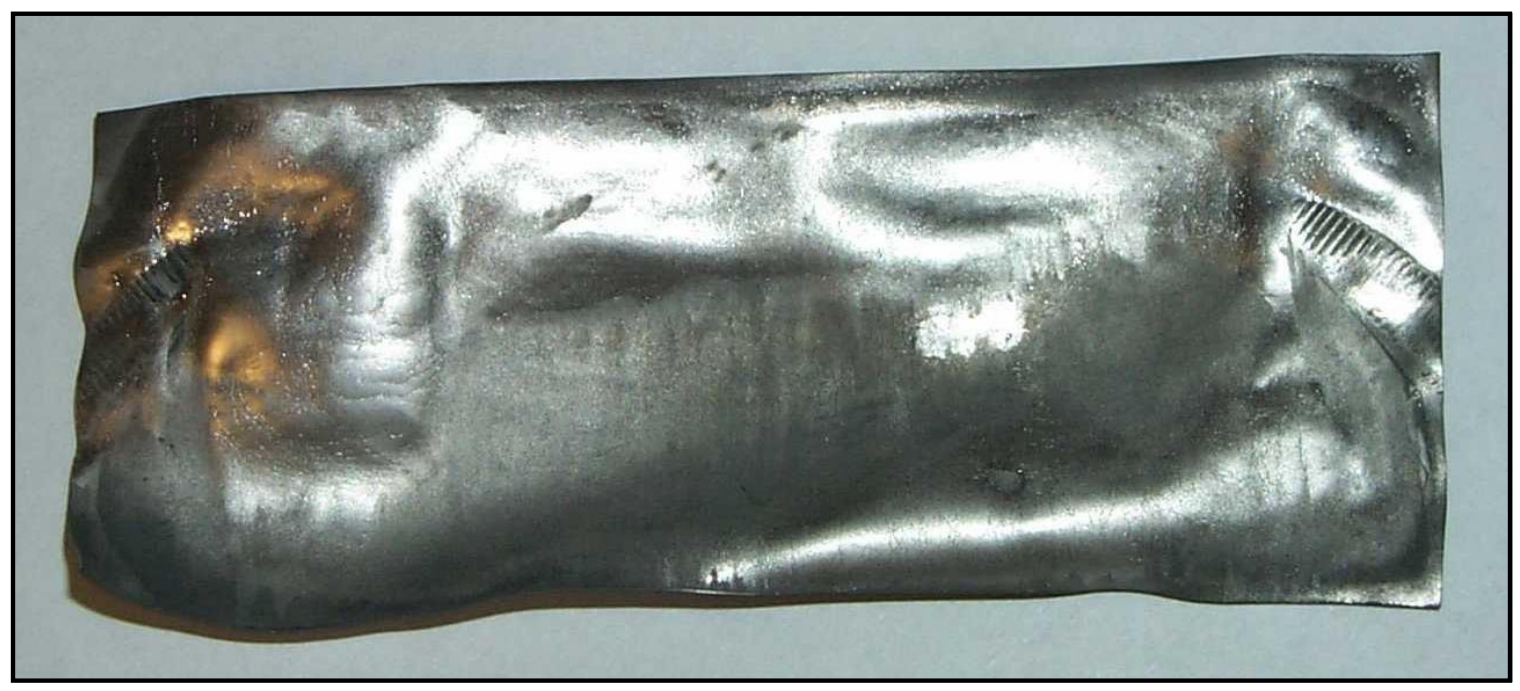

Figure 3.9: Photo of a sample plated with nickel without using the plating fixture. Note that the membrane has become wrinkled and creased during the plating process. 
initial roughening treatment involved sandblasting the membrane with fine glass beads; a later treatment that was investigated involved roughening the membrane by hand using 80 grit sandpaper. This process resulted in a much rougher surface than the sandblasting, and although it did improve the initial adhesion of the nickel to the membrane, the cracking and peeling of the electrodes during actuation was still evident.

When the nickel electrodes flaked off, the underlying membrane had a dark black appearance. This dark black coating seemed very stable and very well adhered to the membrane. At first it was thought that this was the palladium catalyst layer, but X-ray photoelectron spectroscopy (XPS) testing was performed in order to identify the substance. By bombarding a sample with X-rays, the binding energy of the electrons in the sample can be measured. By comparing the spectrum of these binding energies to know references, the elements and compounds in the sample can be identified. Using this technique, the black compound on the surface of the nickel-plated Nafion ${ }^{\mathrm{TM}}$ membranes was identified as nickel oxide. See Figure 3.10 for a plot of the measured binding energy spectrum of the sample tested. Figure 3.11 shows reference spectra of nickel and nickel oxide. Note that the measured spectrum very closely matches the reference spectrum for nickel oxide, although there are some small deviations. During the actual testing of the sample, $285 \mathrm{eV}$ was used as the hydrocarbon reference; in the reference data $284.6 \mathrm{eV}$ is used. This and other dissimilarities in the testing procedure may account for the differences between the measured and reference spectra. It is clear that the measured spectrum corresponds to a surface coating of nickel oxide on the sample, however.

This data indicates that the nickel plated electrodes were oxidizing upon actuation of the samples. This is of much concern for several reasons. First, nickel oxide is not conductive, so when the oxide forms the electrodes lose their conductivity and charge cannot flow into the electrodes, thus the actuator becomes inneffective. Second, the formation of an oxide layer is typically associated with the generation of a stress in the oxidizing layer (7). It is also worthy to note that this is a likely explanation for the cracking and flaking off of the nickel electrodes when the samples were actuated. The instability of non-precious metals as electrodes for ionic polymer actuators has been reported by other researchers, as well. Shahinpoor observed the degredation of copper coatings that had been plated onto Nafion $^{\mathrm{TM}}$ membranes previously plated with gold (53). Le Guilly has observed the same phenomenon when copper was electroplated onto platinum-plated Nafion ${ }^{\mathrm{TM}}$ membranes 


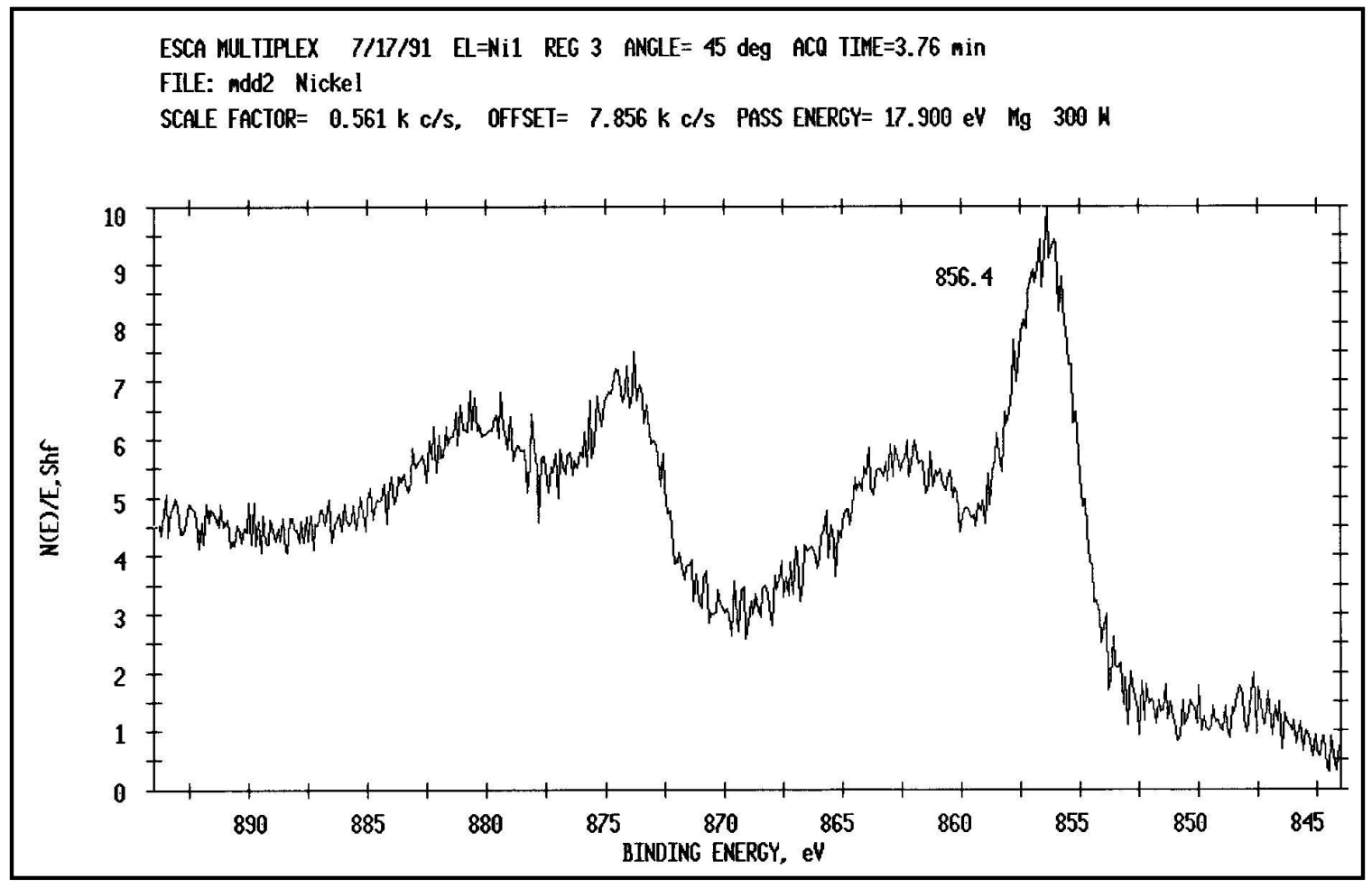

Figure 3.10: Measured XPS data of a nickel-plated sample that had experienced some corrosion of the nickel electrodes.

(24). It is evident that the hydrated and acidic nature of the Nafion ${ }^{\mathrm{TM}}$ represents a highly corrosive environment, further complicated by the fact that applying a voltage across the plated membranes drives the oxidation reaction.

\subsection{Impregnation/Reduction of Copper}

Based upon the results with the gold sputtering and electroless nickel plating processes, a third process was developed that involved the interpenetration of the metal electrode into the polymer matrix, much like that used by other researchers. It was felt that the interpenetration of the electrode and the polymer could overcome the adhesion issues encountered with the other two processes. Further, it is commonly believed that increasing the capacitance of the plated membrane will improve its performance. Several researchers have shown that the capacitance can be increased by increasing the interfacial area between the electrode and the polymer, and thus the charge density on the surface of the membrane $(41 ; 43)$. Attempts were made to increase this interfacial area with the other two processes, primarily by increasing the surface roughness of the membrane prior to plating. The im- 


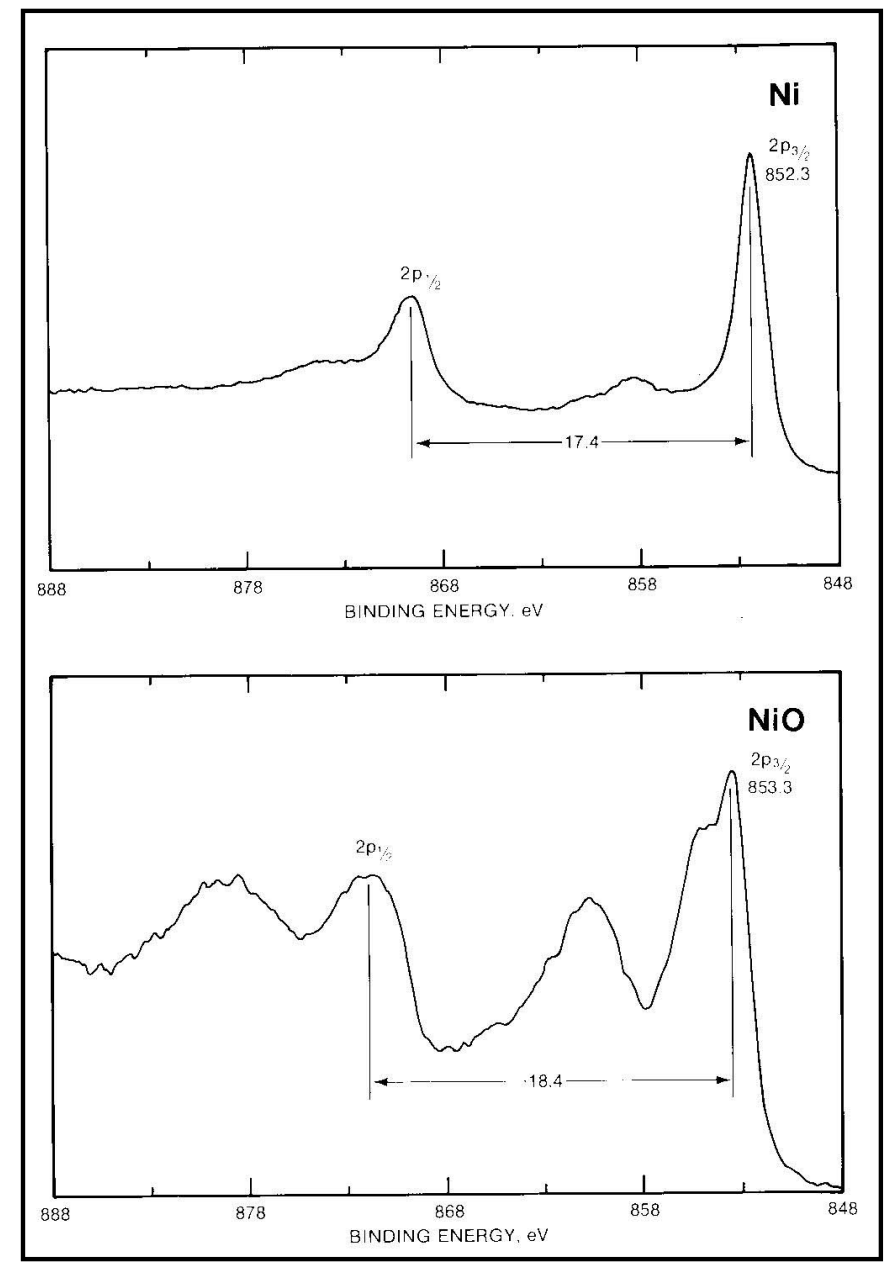

Figure 3.11: Reference XPS spectra of nickel and nickel oxide (60).

pregnation/reduction process is designed to accomplish this goal by allowing the electrode to grow dendritic-like "fingers" into the membrane, enabling a very high charge density, if the conductivity of the electrode is maintained.

Investigation into the available literature revealed that primarily gold and platinum had been used as the conductive electrodes in the work being done on ionic polymer actuators. However, further investigation revealed that researchers in other fields, particularly electrochemistry, had demonstrated success in plating Nafion ${ }^{\mathrm{TM}}$ membranes with many other metals, and that most of this work was being done using impregnation/reduction techniques. For example, Dewulf and Bard and Cook et al. have both demonstrated methods to electrode Nafion membranes with copper by dissolving copper ions into the membrane and reducing them in situ $(10 ; 9)$. Their work focused on the use of these membranes to perform the electrochemical reduction of $\mathrm{CO}_{2}$. More recently, Chen and Chou have plated 
nickel, lead, copper, and silver onto Nafion by a similar method for the reduction of benzaldehyde (8). Based on these findings, a process was developed to plate Nafion membranes with interpenetrating copper electrodes for the purpose of creating ionic polymer membrane transducers.

\subsubsection{Approach}

The process for plating copper electrodes onto Nafion ${ }^{\mathrm{TM}}$ membranes was derived from the work done by Chen and Chou in 1993. The first embodiment of this new process was realized when a sample was plated with copper by first boiling it in $2.0 \mathrm{M} \mathrm{HCl}$ for 15 minutes to wash and hydrate the membrane and to fully saturate it with protons. It was then soaked in $10 \%$ (wt.) $\mathrm{CuSO}_{4}$ for two hours at $40{ }^{\circ} \mathrm{C}$. Following the ion-exchange, the membrane was rinsed and soaked in $1.5 \% \mathrm{NaBH}_{4}$ at $30{ }^{\circ} \mathrm{C}$ for a few minutes. Upon addition to the sodium borohydride solution, a copper deposit could immediately be seen forming on the surfaces of the membrane; the surface resistance of the copper coating was measured to be $4 \Omega / \mathrm{cm}$. Strips of this copper-plated membrane were cut and actuated in the cantilever configuration. Upon application of a voltage across the thickness of these strips, bending of the membrane was observed, indicating that the copper plating is effective as an electrode for an ionic polymer transducer. However, as the strips were continuously actuated, the copper electrodes began to change to a black color. After storing in de-ionized water overnight, the samples had become a blue/green color and the surface resistance had increased to 250 $\mathrm{k} \Omega / \mathrm{cm}$. In their study, Chen and Chou found that in order to improve the stability of their gold, platinum, and copper deposits, the $\mathrm{pH}$ of the reducing agent $\left(\mathrm{NaBH}_{4}\right)$ should be increased to $12-14$ by addition of $\mathrm{LiOH}(8)$. Their explanation was that by increasing the $\mathrm{pH}$ of the reducing agent, the rate of metal deposition was decreased, resulting in a more stable deposit. With this in mind, a new sample was fabricated. First, the sample was pretreated by roughening the surfaces, washing in an ultrasonic cleaner, and boiling, this time in $\mathrm{H}_{2} \mathrm{SO}_{4}$. Copper ions were then exchanged into the membrane by soaking in $10 \%$ (wt.) $\mathrm{CuSO}_{4}$ at room temperature for four hours. The sample was then rinsed under de-ionized water and placed in a solution of sodium borohydride and ammonium hydroxide at room temperature for a couple of minutes, with agitation by a magnetic stirrer, after which time the membrane had become a uniform dark brown color. The surface resistance

of this dark brown coating was measured to be around $200 \mathrm{k} \Omega / \mathrm{cm}$. It is possible that the 
dark brown coating observed in this experiment is a precipitate of copper hydroxide, which is insoluble in water.

Following this experiment, several different iterations on the copper plating process were attempted in an effort to improve the stability of the copper coating, most of them involving some change to the reduction process. Sodium hypophosphite was investigated as a possible reducing agent with no success. Also tried were solutions of sodium borohydride at various temperatures and $\mathrm{pH}$. All of the samples plated in these experiments had a dark brown appearance and very high surface resistance (greater than $100 \mathrm{k} \Omega / \mathrm{cm}$ ). This dark brown substance was later identified by XPS testing to be copper oxide. Like nickel oxide, copper oxide is not a good electrical conductor, and the formation of copper oxide on the Nafion $^{\mathrm{TM}}$ membrane renders it useless as a transducer.

Following the XPS testing more experimentation was performed, again with little success, but an interesting phenomenon was observed. During the reduction process, the portion of the membrane near the clamp that was holding it visually appeared to be mettalic copper, whereas the rest of the deposit appeared to be copper oxide. Because the portion of the membrane near the clamps experiences a low mass transfer rate of reactants relative to the rest of the membrane, it was reasoned that greatly lowering the concentration of the reducing agent might yield better results. Copper platings were deposited using sodium borohydride as the reducing agent in concentrations of $0.01 \%-0.5 \%$ (wt.) with much better results. Based on these new experiments, a concentration of sodium borohydride of $0.08 \%$ (wt.) was found to result in high-quality copper coatings - see Figure 3.12 for a photo of a sample plated with copper. It seems that by decreasing the rate of the reduction reaction, the deposited coating is copper, whereas faster deposition rates result in coatings of copper oxide. This supports the findings of Chen and Chou that reducing the rate of metal deposition results in a more stable coating. Unfortunately, the same problem encountered when using the electroless nickel plating process was encountered again using this impregnation/reduction copper plating process - the electrodes oxidized when the samples were actuated.

Although other researchers have used impregnation/reduction techniques to deposit copper electrodes onto Nafion ${ }^{\mathrm{TM}}$ membranes, these efforts have focused on the study of the electrochemical characteristics of the copper/Nafion ${ }^{\mathrm{TM}}$ composites. Only a few investigators have employed copper as an electrode material in an ionic polymer electromechanical trans- 


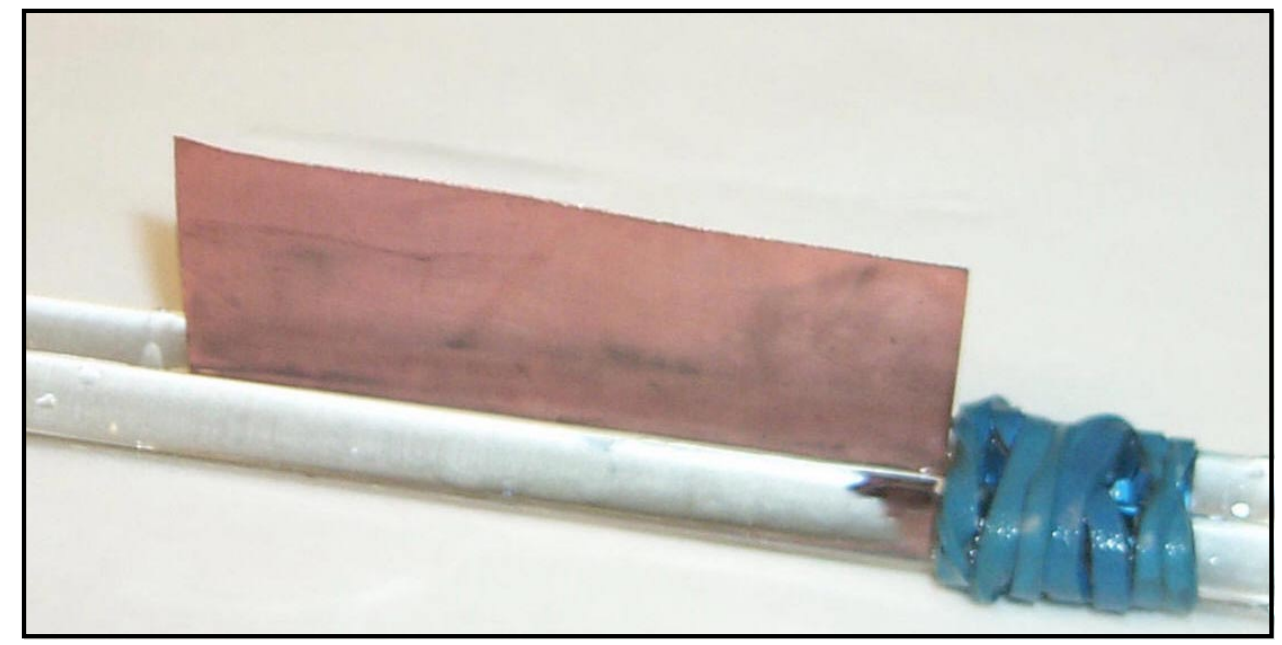

Figure 3.12: Photo of a sample of Nafion ${ }^{\mathrm{TM}}$ membrane plated with copper.

ducer $(24 ; 53)$. In these reports, a copper overlayer was electroplated over an initial layer of gold or platinum. The gold or platinum layer was deposited by an impregnation/reduction technique, and these researchers reported that the copper coatings corroded during actuation of the membranes. The current work represents the first time that copper electrodes have been deposited onto ionic polymer membranes using an impregnation/reduction technique and the resulting composites characterized as electromechanical transducers.

Chen and Chou have also reported instability of their lead, copper, and nickel electrodes during the electrochemical reduction of benzaldehyde. They found that electrodes that contained noble metals were more stable than those that did not. Once a process was developed to quickly deposit stable copper coatings onto Nafion ${ }^{\mathrm{TM}}$ membranes, the focus of the work was shifted to study ways of improving the stability of the copper electrodes during actuation. As a first attempt, a process for depositing thin layers of $24 \mathrm{kt}$ gold over the copper electrodes was investigated. This process, sold by Alfa Aesar as part number 42307, consists of a single-part electroless plating bath that operates at elevated temperature and will deposit a thin gold layer onto a copper or nickel substrate at a rate of 0.005-0.007 microns per minute up to a maximum thickness of about 40 nanometers (2). Using this electroless gold plating process, high-quality gold deposits were plated onto the copper electrodes on a hot plate. These gold platings had the benefit of improving the surface conductivity of the electrodes from about $3-4 \Omega / \mathrm{cm}$ to less than $1 \Omega / \mathrm{cm}$. Also, whereas the copper electrodes that were not subsequently plated with gold would oxidize in less than 12 hours if simply stored in de-ionized water, the addition of the gold coating allowed for 
seemingly indefinite storage without visible degredation of the electrode. Chen and Chou have used a similar approach to plate protective layers of platinum by an electroless plating method over lead/palladium electrodes deposited by an impregnation/reduction procedure (8).

Also fundamental to the deposition of high-quality copper coatings was the development of a new type of plating fixture that tensioned the membrane in two directions. This fixture was effective in holding the membrane flat throughout the plating process and its use improved the quality of metal coatings - please refer to Figure 3.13 for a photo of this fixture. In Figure 3.13a, the fixture is shown held in a clamp, with a template that was used to cut the membrane sample to the proper size. The membrane is then secured to the fixture with a screw in each corner and the fixture removed from the clamp. This allows the springs in the fixture to apply a tension to the membrane and to compensate for the volume change that results from hydrating the membrane. A sample of the membrane in the fixture can be seen in Figure 3.13b.

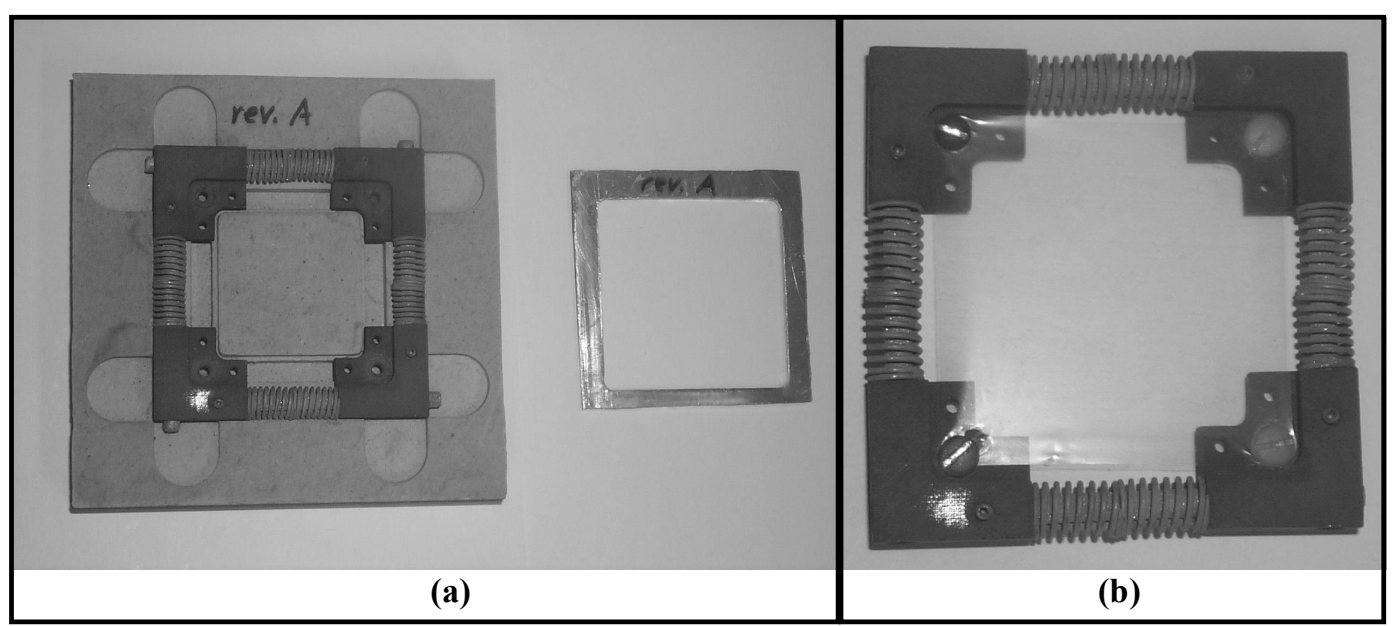

Figure 3.13: (a) Photo of the fixture in a clamp, with a template used to cut the membrane to the correct size. (b) Photo of a membrane sample in the fixture

\subsubsection{Results}

The results with this process showed that the electromechanical coupling was much more pronounced than in the samples plated using the gold sputter-coating or nickel electroless plating processes. Figure 3.14 shows the peak force/deflection plot of a sample of Nafion ${ }^{\mathrm{TM}}$ plated with copper and an overlayer of gold using the process described above; this plot 
was generated immediately after the plating process. This sample was $5 \mathrm{~mm}$ wide with a $17 \mathrm{~mm}$ free length and the input was a $1.0 \mathrm{~V}$ step. Note that although when the sample is new it is capable of producing forces and deflections that compare well to the baseline material, oxidation of the copper electrodes will cause a significant reduction in the work output of this device.

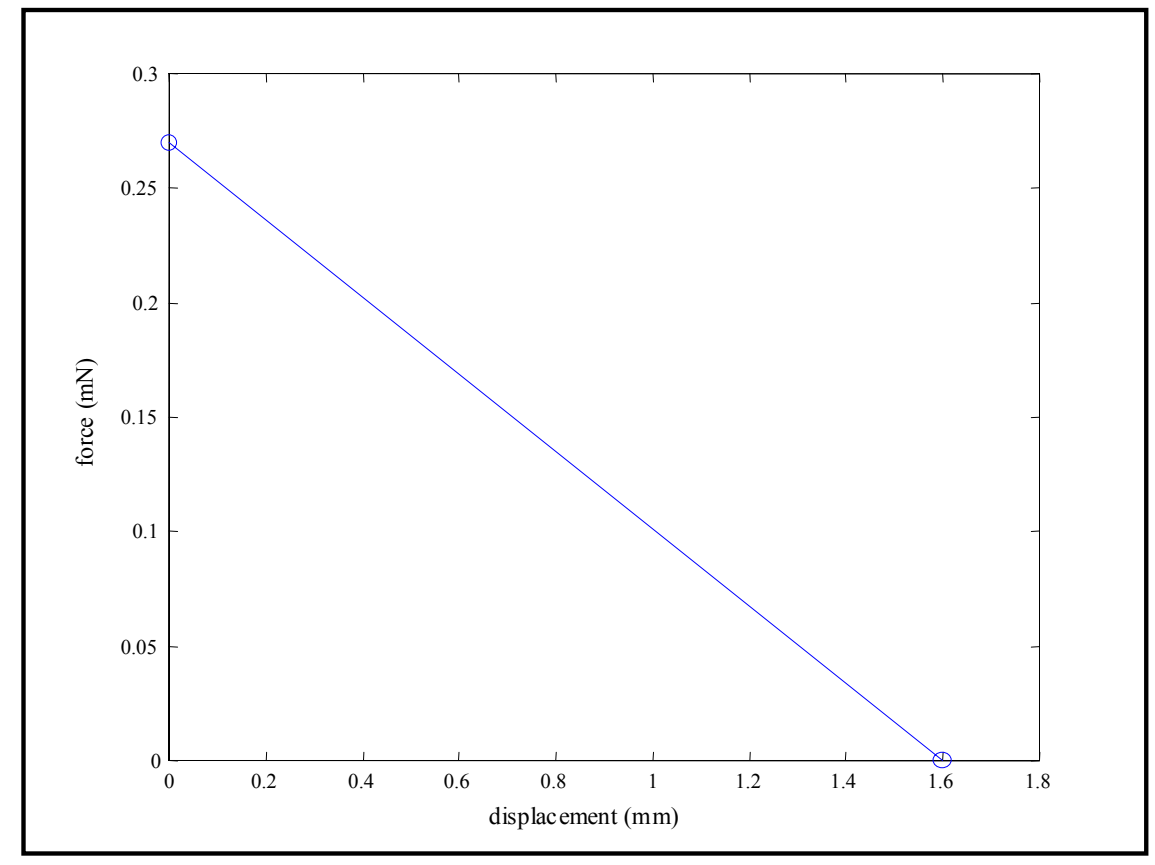

Figure 3.14: Force/deflection curve for a sample of Nafion ${ }^{\mathrm{TM}} 5 \mathrm{~mm}$ wide with a $17 \mathrm{~mm}$ free length that was plated with copper with a gold overlayer. The input was a 1.0 V step and the test was performed immediately after plating. Note that the plot was generated using only the blocked force and free deflection.

Refer to Figures 3.15 and 3.16 for micrographs of these electrodes. These images show that the copper layer extends about 0.6 microns into the membrane and that the gold overlayer is about 50 nanometers thick. This interpenetration of the metal and the polymer explains the improved electromechanical coupling observed in these materials as compared to the samples plated using the gold sputtering or electroless nickel plating processes. The interpenetrating electrodes provide a higher charge density at the surface of the membrane. This interpenetration is also responsible for the improved adhesion of these electrodes.

Once a method for depositing copper electrodes with a gold overlayer was developed, a further study of the stability of devices made by this process was initiated in an attempt to identify the root causes of the instability of the electrodes and develop ways to improve this stability. 


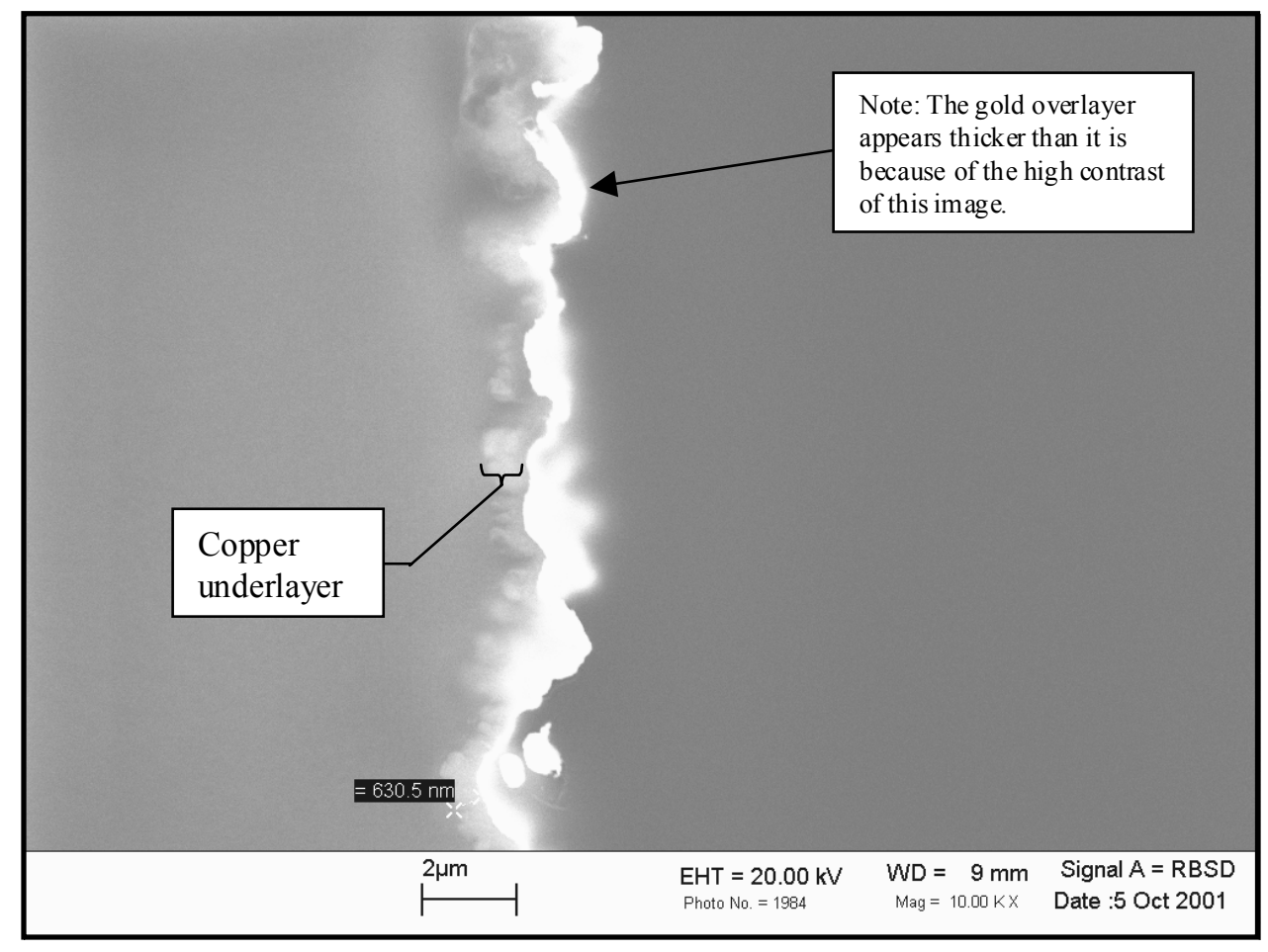

Figure 3.15: Scanning electron micrograph showing the penetrating copper layer and the gold overlayer. Note that the copper layer is about 0.6 microns thick.

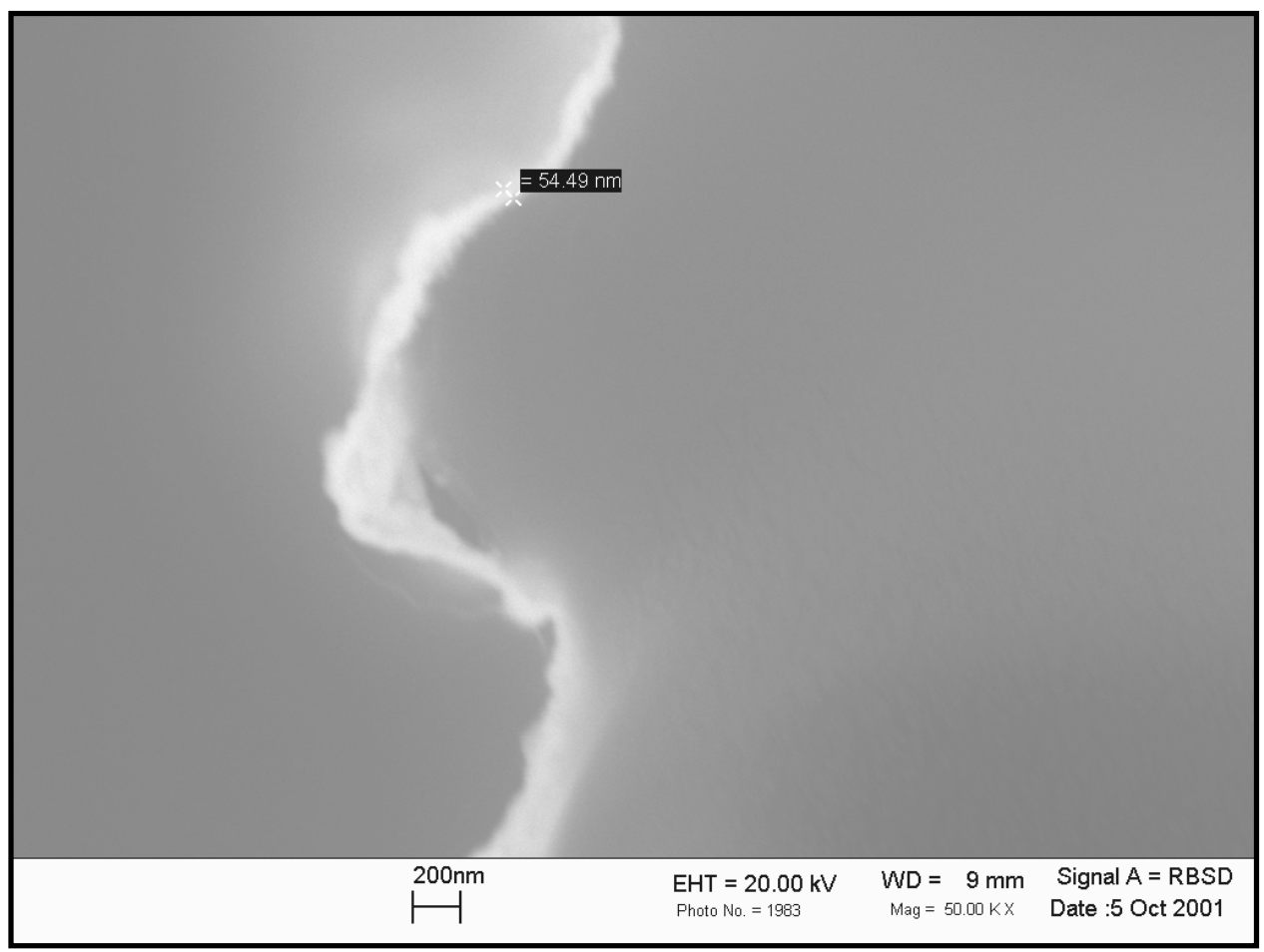

Figure 3.16: Micrograph showing that the gold layer is about 50 nanometers thick. The copper layer is not visible due to the low contrast of the image. 


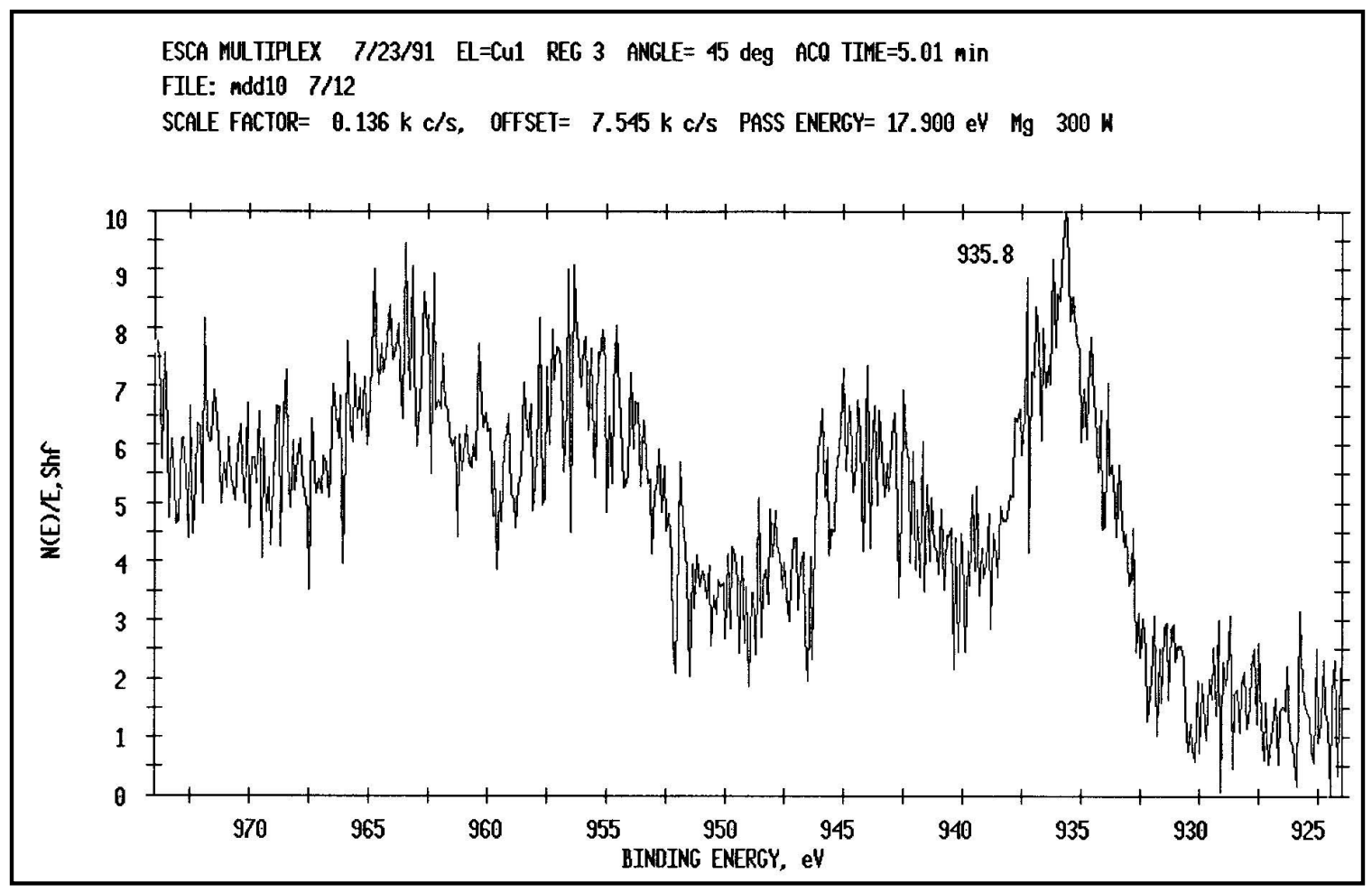

Figure 3.17: Measured XPS spectrum of the surface of a copper-plated sample that had undergone some corrosion.

As mentioned, the copper electrodes were found to oxidize upon actuation. The oxidation of the electrodes was confirmed by XPS testing. See Figure 3.17 for a plots of the measured XPS spectrum of the oxidized copper-plated samples. Figure 3.18 shows reference spectra of copper and copper oxide. Although there is a great deal of noise in the measured data, four distinct peaks can clearly be seen in Figure 3.17, indicating that the substance present on the surface of the membrane is copper oxide. Once the nature of the degredation of the copper was confirmed to be oxidation, future oxidation could be identified simply by observing the drastic color change that occurs in the electrodes during oxidation.

As mentioned, the purpose of the gold layer was to prevent oxidation of the copper electrodes. Unfortunately, the gold overlayer had little effect on the stability of the copper electrodes under actuation conditions. Longevity testing was performed as described in Chapter 2 to characterize the stability of the electrodes. As can be seen in Figure 3.20, the samples that were treated with the gold overlayer experience a dramatic decrease in their measured free tip deflection upon continuous cyclic actuation, just as those without the gold overlayer do. As before, the oxidation of the copper is associated with a color change in the 


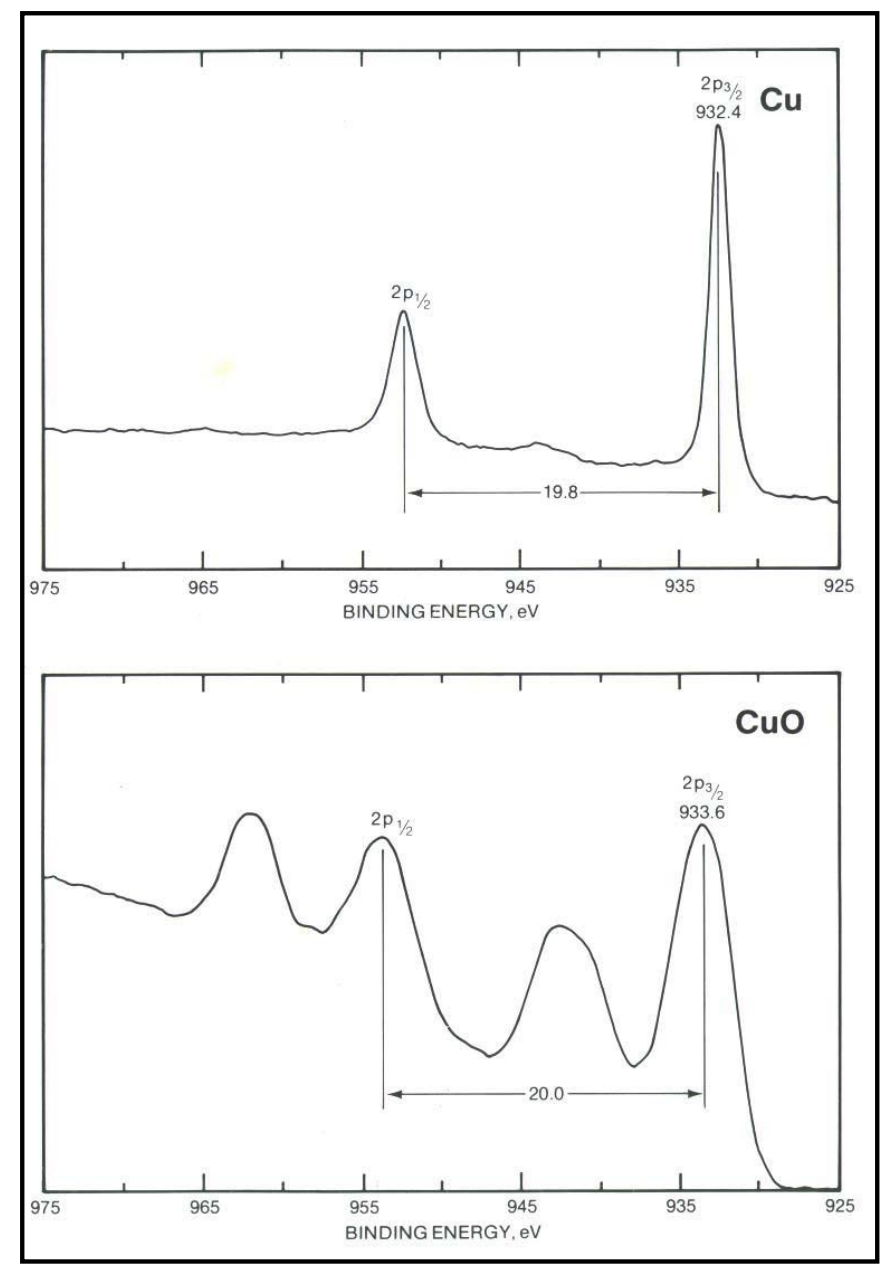

Figure 3.18: Reference XPS spectra of copper and copper oxide (60).

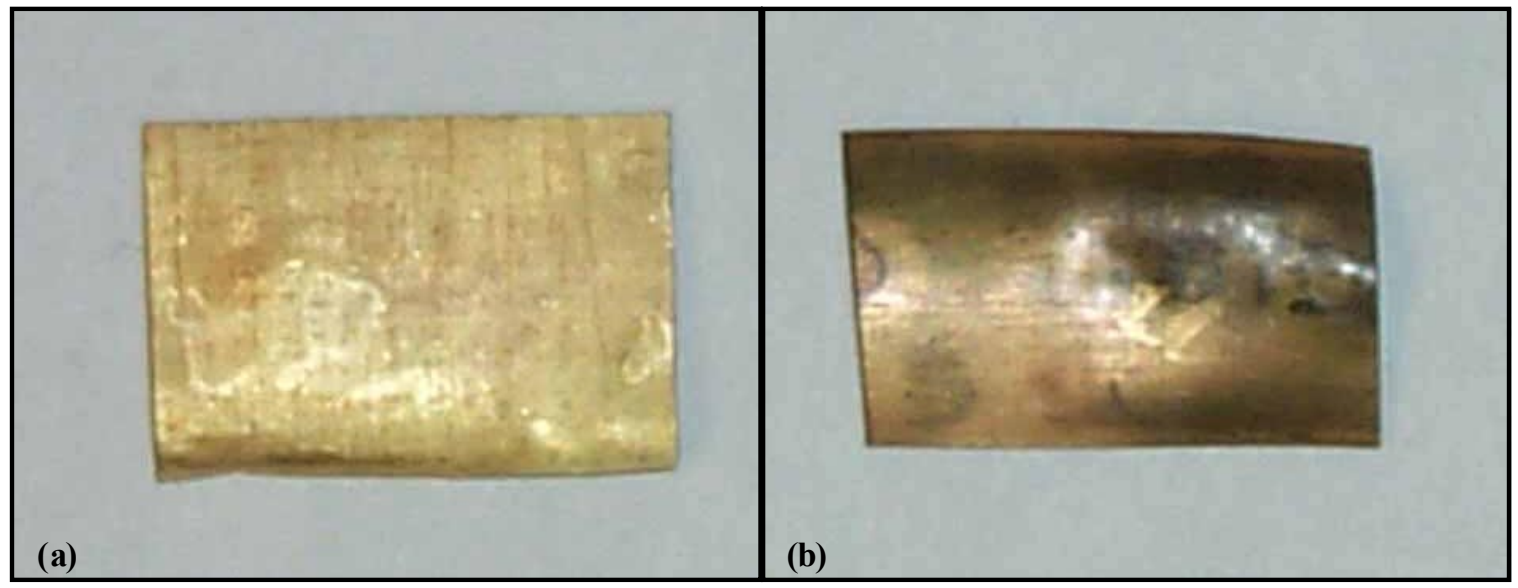

Figure 3.19: Photos of samples of Nafion ${ }^{\mathrm{TM}}$ membrane plated with copper with a gold overlayer (a) before oxidation of the copper layer, and (b) after oxidation of the copper layer. 


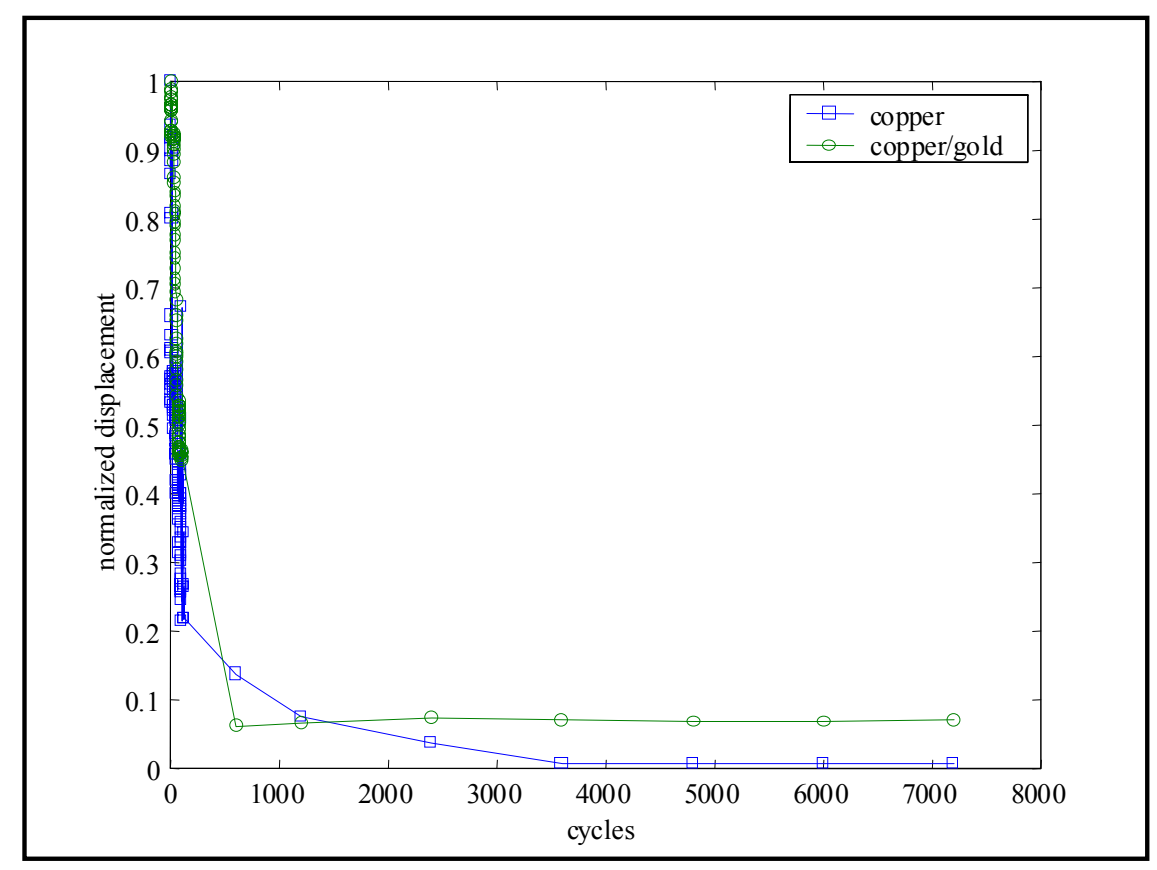

Figure 3.20: Normalized tip displacement versus the number of actuation cycles of a strip of Nafion ${ }^{\mathrm{TM}}$ plated with copper, and a strip plated with copper with a gold overlayer. The input was a $1.5 \mathrm{~V}, 1.0 \mathrm{~Hz}$ sine wave.

electrode. This color change is evident even when viewed through the gold overlayer, further indicating that the gold layer is very thin. See Figure 3.19 for photos of a sample plated with copper and gold before and after oxidation of the copper layer. It is interesting that although the samples without the gold layer exhibit no measurable deflection after a period of 3500 cycles or so, the tip deflection of the samples with the gold layer seems to drop to a small but steady value. This is likely because the initially deposited copper layer becomes fully oxidized, but the gold overlayer remains unaffected during the continuous actuation. Thus, even when the copper electrode has become fully ineffective, the gold still serves to transfer some charge down the length of the ionic polymer strip. This is further support for the idea that increased interfacial area between the electrode and the polymer membrane affords better performance of the composite. At the beginning of the longevity test, the copper layer interpenetrates the polymer membrane and provides a very charge density at the membrane/metal interface. Once the copper layer oxidizes and loses its conductivity, the effective interfacial area becomes very small and the only condutive electrode is the gold overlayer, and the free tip deflection of the sample drops to less than one tenth of its original value. This situation is analagous to the sputtered gold electrodes discussed in Section 3.3 
above. Thus, although the addition of the gold layer did improve the stability of the copper electrodes under storage conditions, it did not improve the stability of the copper electrode when the sample was actuated. Following this result, several other tests were run in an attempt to identify the root causes of the oxidation of the copper electrodes.

It follows to reason that oxidation is caused by the presence of oxygen, and to this point no attempt had been made to decrease or control the amount of oxygen present in either the solutions used to perform the plating or the water that the samples were actuated in during the longevity testing. For this reason, a new test was performed. Immediately after the plating process was completed, the samples were cut and mounted in a cantilevered configuration and placed into a bath of de-ionized water. Pure nitrogen was then bubbled through the water bath continuously for two hours. A cover was then placed over the bath and nitrogen gas was flowed through the empty space between the top of the water and the cover. Figure 3.21 shows the normalized free tip deflection versus the number of actuation cycles for the four samples run simultaneously during this test. Note that although extensive measures were untertaken to remove all oxygen from the testing environment, the free tip deflection of the samples had dimished to less that $10 \%$ of its original value within 1200 actuation cycles.

Based upon this result, it seems possible that the cause of the oxidation of the copper electrodes upon actuation may be oxygen dissolved in the Nafion ${ }^{\mathrm{TM}}$ membrane, and not oxygen dissolved in the water bath. In an attempt to reduce the amount of oxygen dissolved in the membrane, a new test was devised in which nitrogen was bubbled through all of the aqueous solutions that the membrane encountered during the plating process. The nitrogen bubbling was begun at least 30 minutes prior to the membrane being placed in any solution. Following the plating procedure, the membrane was immediately placed into a bath of mineral oil and three strips were measured and cut from the larger sample. These strips were then mounted in a cantilevered configuration and actuated continuously with a 1.25 V, $1.0 \mathrm{~Hz}$ sine wave as the free tip deflection of each sample was periodically recorded. The testing was done in a bath of mineral oil to prevent dehydration of the samples. Please refer for Figure 3.22 for the results of this test. As before, the tip deflection of all of the samples drops to less than $10 \%$ of its initial value within 3000 actuation cycles.

One reason for this result may be the relatively high oxygen solubility of Nafion ${ }^{\mathrm{TM}}$. Ogumi et al. have studied the solubility and diffusion constants of hydrogen and oxygen gas 


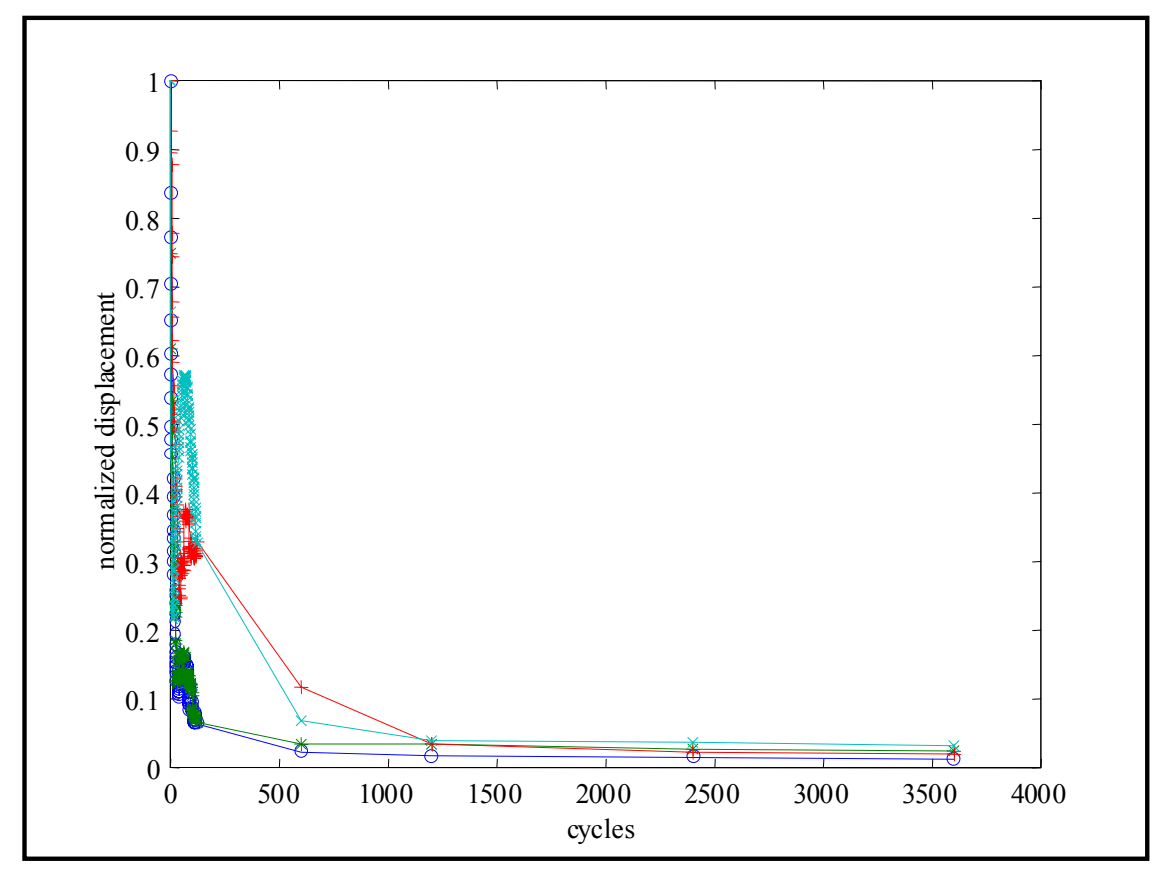

Figure 3.21: Normalized tip displacement versus the number of actuation cycles of four strips of Nafion ${ }^{\mathrm{TM}}$ plated with copper with a gold overlayer. The input was a $1.25 \mathrm{~V}, 1.0 \mathrm{~Hz}$ sine wave. Nitrogen gas was bubbled through the water bath for 2 hours prior to testing and the space above the bath was flushed with nitrogen during testing.

in Nafion ${ }^{\mathrm{TM}}$ of varying exchange capacities and water contents and with various counterions (38). Thier findings indicate that the solubility of oxygen gas in Nafion ${ }^{\mathrm{TM}}$ is on the order of $10^{-2}$ mol per cubic decimeter and that this value was essentially independent of the water content, exchange capacity, or counterion in the membrane. Based on this information, it seems possible that there is a large amount of oxygen within the membrane as-recieved and that elaborate processing will be required to remove it.

\subsection{Summary}

In the initial stages of this research project, three plating techniques were investigated: gold sputter-coating, electroless nickel plating, and impregnation/reduction plating of copper. The results of these processes demonstrated that electromechanical transduction in hydrated Nafion $^{\mathrm{TM}}$ membranes may take place if a conductive electrode is plated onto the surfaces of the membrane. These results indicate that the use of a noble metal and the interpenetration common with most of the traditional techniques are not necessary for this transduction to 


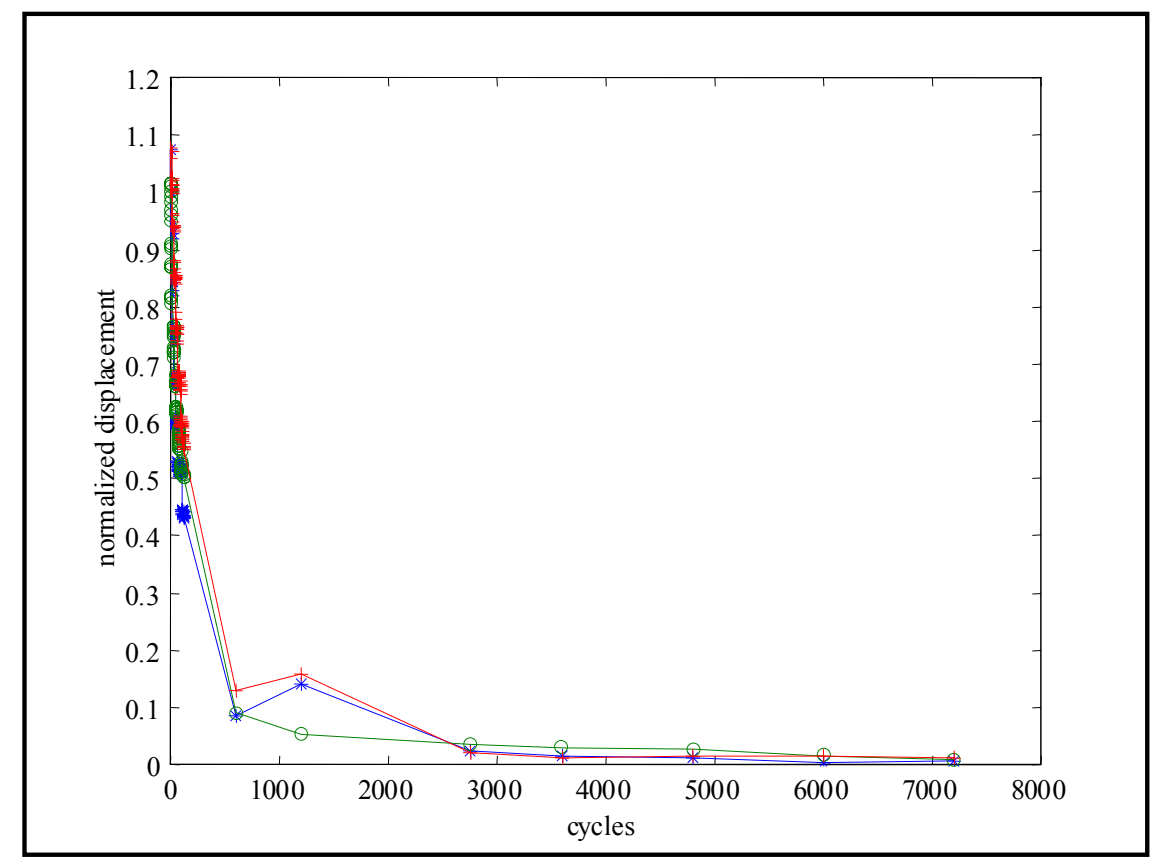

Figure 3.22: Normalized tip displacement versus the number of actuation cycles of three strips of Nafion ${ }^{\mathrm{TM}}$ plated with copper with a gold overlayer. The input was a $1.25 \mathrm{~V}, 1.0 \mathrm{~Hz}$ sine wave. Nitrogen gas was bubbled through all solutions during the plating process. The samples were actuated in a bath of mineral oil.

occur. Several key issues associated with these processes were identified, however.

When using a plating process that does not result in an interpenetrating electrode, significant adhesion problems were encountered. Typically, the electrodes made in this way would flake off during actuation of the samples, or would rub off of the membrane during handling. These adhesion problems are likely caused by the Teflon ${ }^{\mathrm{TM}}$-like properties of the Nafion ${ }^{\mathrm{TM}}$ membrane, and are compounded by the water within the membrane and the fact that the membrane undergoes large volume changes upon hydration. In order to overcome these adhesion problems, an impregnation/reduction process was developed.

Another key issue with the processes is the oxidation of non-noble metals upon actuation. When electrodes are made using non-noble metals, the inherent instability of these metals allows for quick oxidation of the electrodes during actuation of the membrane. This oxidation causes a large increase in the surface resistance of the electrodes, resulting in a decrease in the charge capacity of the plated device. In some cases, this oxiation also causes the electrode to become de-bonded from the Nafion ${ }^{\mathrm{TM}}$ membrane. While some efforts were undertaken to prevent this oxidation by the addition of a protective gold layer to the electrode and removal of dissolved oxygen from the region of the electrode, these 
efforts did not succesfully affect the oxidation problem.

The following chapters will discuss other approaches to overcoming the problem of electrode oxidation and the results of these efforts, as well as a discussion of the characterization of the properties and performance of the ionic polymer transducers made using these new processes. 


\section{Chapter 4}

\section{Description and Results of Co-Reduction Method}

\subsection{Introduction}

Chapter 3 discussed some novel methods of plating both noble and non-noble metals onto Nafion $^{\mathrm{TM}}$ membranes and identified some of the issues associated with these plating processes. By exploring plating methods that do not involve the polymer/metal interlocking associated with most of the processes currently employed by other researchers, it has been shown that this interlocking is not essential for electromechanical transduction to take place. Without this interlocking, significant adhesion problems were encountered, however. Also, the key issue associated with the use of non-noble metals as the electrode in ionic polymer transducers has been identified as oxidation of the electrode. Although extensive efforts to eliminate this oxidation have been undertaken, so far the results have not been succesful. In this chapter, new methods of forming electrodes on ionic polymer membranes are discussed, and the results of these methods and their effect on the oxidation of the electrodes are presented. Also, a detailed investigation of the mechanical properties of these new devices is presented, including measurements of the useful energy density and comparisons to a baseline material. 


\subsection{Concept of Co-Reduction}

Of the three methods for plating electrodes onto Nafion ${ }^{\mathrm{TM}}$ membranes that were discussed in Chapter 3, the most promising is the impregnation/reduction of copper. This process resulted in membranes that produced more deflection and at lower voltages than the gold sputtering or electroless nickel plating processes and also overcame the adhesion problems inherent with those processes. The copper electrodes were found to oxidize upon actuation of the samples, however. In order to increase the stability of the copper, a new process has been developed that involves the co-reduction of copper and platinum at the membrane surface as an electrode.

The concept of co-reduction is not a new one. In 1993, Chen and Chou reported on a process to sequentially exchange lead and then palladium ions into Nafion ${ }^{\mathrm{TM}}$ membranes and then simultaneously reduce these ions using a mixture of sodium borohydride and lithium hydroxide (8). Their goal was to find a suitable catalyst layer for the electrochemical reduction of benzaldehyde. They desired to use lead because its hydrogen overpotential is very high, but lead by itself is not stable as an electrode. By co-reducing lead with palladium and then covering this surface with a protective layer of platinum, a stable electrode with efficient operation was acheived. In a similar fashion, Millet et al. have suggested the use of co-reduction to improve the stability of ruthenium anodes in electrolyzer applications (31). Ruthenium anodes have lower anodic overvoltages than platinum anodes, but ruthenium corrodes in this application $(31 ; 59)$. Millet et al. have suggested that co-reducing ruthenium with platinum may lead to the development of more stable ruthenium-based anodes. In this work, Millet et al. developed a model for predicting the rate of ion-exchange of tetraammine platinum ions into Nafion ${ }^{\mathrm{TM}}$ membranes initially in the hydrogen-ion form for different platinum salt concentrations and volumetric flow rates of platinum salt in the ion-exchange cell. They found that a shift from the exchange rate being controlled by diffusion in the boundary layer just outside the membrane to the exchange rate being controlled by diffusion within the membrane occured as the salt concentration and volumetric flow rate were increased. Although no results for multiple ions exchanging with a single membrane were presented, the model could possibly be expanded to include this case if sufficient experimental data were available.

Of course, for two metals to be simultaneously reduced in a Nafion ${ }^{\mathrm{TM}}$ membrane, 
they must first be simultaneously exchanged into the membrane, meaning that an understanding of the ion-exchange properties of Nafion ${ }^{\mathrm{TM}}$ membranes must be gained. Dr. H. L. Yeager, formerly of the University of Calgary, Canada, is as much an expert in this field as anyone. In collaboration with Dr. Steck, also of the University of Calgary, he has extensively studied the ion-exchange selectivities of Nafion ${ }^{\mathrm{TM}}$ and compared them with more conventional cross-linked sulfonated ion-exchange resins. These researchers have determined the selectivity coefficients for exchange of hydrogen ion with various univalent (65) and divalent cations $(54 ; 63)$. The selectivity coefficients were determined by fully exchanging samples of Nafion ${ }^{\mathrm{TM}}$ that were initially in a homoionic form with solutions containing both hydrogen ion and an alkali metal or alkaline earth ion in different relative molarities but with constant ionic strength. The resulting ionic fraction of the alkali metal or alkaline earth ion in the membrane after full exchange was used to determine the selectivity coefficient of that ion to hydrogen ion. The results of their work show that the selectivity coefficient for these various ions can be predicted by considering the amount of water that moves out of the polymer during the exchange. Because Nafion ${ }^{\mathrm{TM}}$ in the hydrogen-ion form is in its most hydrated state, water will always leave it when another ion is exchanged into the polymer. The expulsion of water from the polymer matrix and the resulting contraction of the polymer are both entropy-producing processes, which causes the polymer to prefer any ion over hydrogen. This is notably different than traditional cross-linked sulfonated ion-exchange resins, in which the water content changes very little for different ions. This can be explained by the fact that the non cross-linked nature of Nafion ${ }^{\mathrm{TM}}$ allows for a much larger amount of swelling and thus the entropy change of the exchange and not the electrostatic interactions between the ions and the exchange sites dominates the selectivity of the polymer. While this is very interesting, Yeager and Steck did not study the selectivity coefficient of Nafion ${ }^{\mathrm{TM}}$ to copper ion or any noble metal ion, and thus other results of their work are more important to the current effort. First, they have shown that the number of available exchange sites in a given sample is independent of the exchanging ion $(65 ; 54 ; 63)$. Again, this is likely because the polymer is not cross-linked and thus able to swell such that all of the exchange sites in the polymer are available for exchange, regardless of the size of the exchanging ion. Second, they showed that multiple ions can be exchanged into a sample of Nafion ${ }^{\mathrm{TM}}$ simultaneously, and that the relative proportion of the ions in the membrane can be controlled by changing the relative molarities of the ions in the exchanging solution. 


\subsection{Approach}

In the first attempt at a co-reduction process, the two metals used were copper and gold. As a precursor to this attempt, a membrane sample was deposited with gold from a solution of gold chloride $\left(\mathrm{AuCl}_{3}\right)$.

This gold deposition was carried out as follows. The membrane was pretreated by sanding both sides and washing in an ultrasonic cleaner. It was then boiled in $0.5 \mathrm{M} \mathrm{HCl}$ for 30 minutes to hydrate the membrane and saturate it with protons. Following this, the sample was soaked in $0.1 \%$ (wt.) $\mathrm{AuCl}_{3}$ for about 3 hours to allow the $\mathrm{Au}^{3+}$ ions to exchange into the membrane; the gold solution was stored in a beaker wrapped with aluminium foil to prevent photoreduction of the ions in the solution. The sample was then rinsed with de-ionized water and soaked in $0.08 \%$ (wt.) $\mathrm{NaBH}_{4}$ for about 5 minutes to reduce the gold ions to metallic gold. During the reduction process, a color change was observed in the membrane as it took on a slight yellow tint, but the dense electrodes formed during similar processing with copper were absent. The measured surface resistance of the membrane following this processing was very high, indicating that although a small amount of gold was possibly deposited in the sample, it was not enough to form a conductive electrode.

The next step in this process was to attempt to deposit gold and copper in the membrane at the same time. For this test, a solution of $0.0118 \mathrm{M} \mathrm{CuSO}_{4}+0.0039 \mathrm{M} \mathrm{AuCl}_{3}$ was prepared. As before, the solution was protected from light to prevent photoreduction of the gold ions. Another membrane sample was prepared, by the same pretreatment as before, and soaked in this copper/gold solution overnight. After the ion-exchange, the sample was soaked in a solution of $0.08 \%$ (wt.) $\mathrm{NaBH}_{4}$ for 12 minutes to reduce the metal ions. Because of the inclusion of the copper in the solution, the electrodes formed by this processing had good conductivity and two strips from this sample were cut and tested in a longevity test, the results of which can be seen in Figure 4.1 below.

As can be seen from the figure, the stability of the electrodes made using this technique is no better than those made using any of the other processes, and the free tip deflection of these samples drops to below $1 \%$ of its original value within 1200 actuation

cycles. It may be reasoned from this result that there was not enough gold present in the electrode to affect the electrode stability in a positive manner. It is possible that more dense gold deposits could be obtained by increasing the concentration of gold in the exchanging 


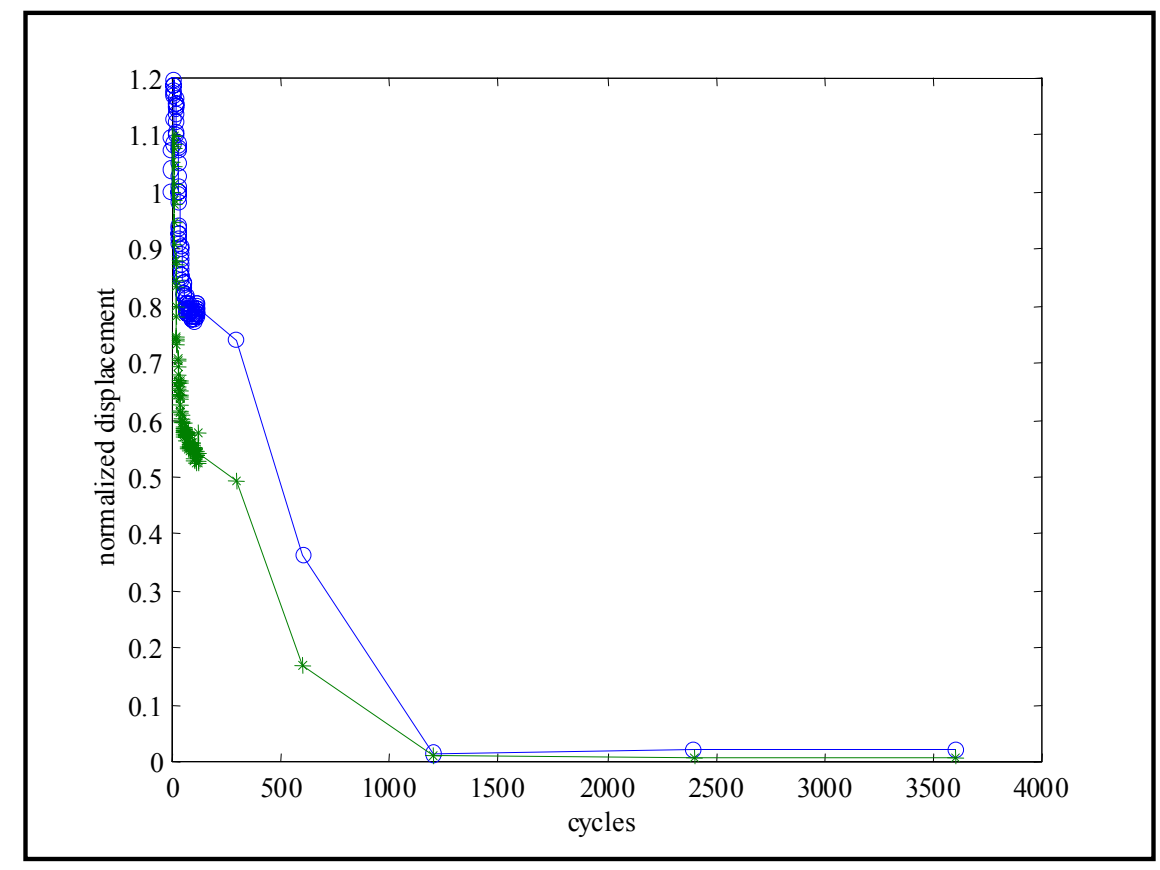

Figure 4.1: Normalized tip displacement versus the number of actuation cycles of two strips of Nafion ${ }^{\mathrm{TM}}$ plated with an alloy of copper and gold. The input was a $1.25 \mathrm{~V}, 1.0 \mathrm{~Hz}$ sine wave.

solution.

In another attempt to make a copper/gold alloy, the deposition of gold and copper was done sequentially, with much better results. A sample of Nafion ${ }^{\mathrm{TM}}$ membrane was pretreated, again by sanding, ultrasonic cleaning, and boiling in $0.5 \mathrm{M} \mathrm{HCl}$, and was then soaked in $0.85 \%$ (wt.) $\mathrm{AuCl}_{3}$ for about 48 hours. This sample was then rinsed with deionized water and the gold ions were reduced in a $0.15 \%$ (wt.) solution of $\mathrm{NaBH}_{4}$ for about 9 hours. Following this treatment, the sample was soaked in $0.5 \%$ (wt.) $\mathrm{CuSO}_{4}$ for 2 hours and then in $0.08 \%$ (wt.) $\mathrm{NaBH}_{4}$ for a few minutes. The electrode formed in this way was then plated over with a thin layer of gold using the electroless gold plating solution described in Chapter 3. This processing presumably resulted in an electrode that consisted of gold and copper phases just below the membrane surface, covered by a thin layer of pure gold outside the membrane. Longevity testing on a strip of this sample was conducted, with the results shown in Figure 4.2 below. Please note that for this test the input signal was a 3.0 $\mathrm{V}$ sine wave at $1.0 \mathrm{~Hz}$, not the typical $1.25 \mathrm{~V}$ or $1.5 \mathrm{~V}$ sine wave input of most of the other longevity tests. This particular sample was found to exhibit relatively low electromechanical coupling and the free displacement response was very small at lower voltage levels. The 


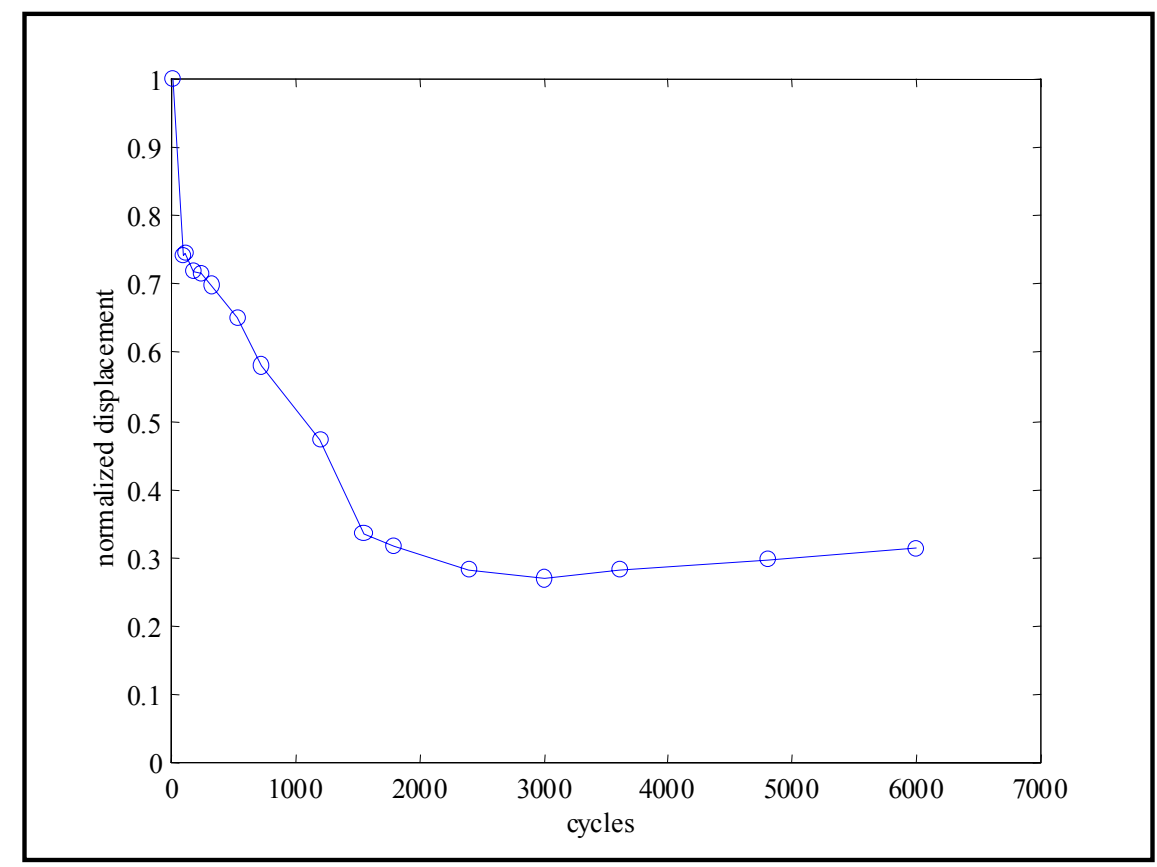

Figure 4.2: Normalized tip displacement versus the number of actuation cycles of a strip of Nafion ${ }^{\mathrm{TM}}$ deposited with gold and then with copper and subsequently electroless plated with an overlayer of gold. The input was a $3.0 \mathrm{~V}, 1.0$ $\mathrm{Hz}$ sine wave.

specific reasons for this are unknown.

Although the electromechanical coupling in this material was smaller than that observed in other samples, it did have improved stability over these other samples. Even after 6000 cycles with a $3.0 \mathrm{~V}$ actuation input, the free tip displacement is still more than $30 \%$ of its original value. This seems to indicate that there is some hope for improvements to the stabiltiy of non-noble metal electrodes by the addition of small amounts of noble metals as alloying metals. The current method of depositing gold clearly suffers from some problems, however. Although the idea seems sound, after several attempts at using gold chloride to deposit gold electrodes onto Nafion ${ }^{\mathrm{TM}}$ membranes using an impregnation/reduction procedure, it is clear that fundamental limitations exist either in the ion-exchage process or in the reduction process. After a thorough search, no other source of gold cations was found to be commercially available. In order to overcome this problem, a new treatment was developed using platinum as an electrode material.

The deposition of platinum was first realized when a sample of Nafion ${ }^{\mathrm{TM}}$, pretreated in the usual way, was soaked in $0.1 \%$ (wt.) tetrammine platinum chloride $\left(\left[\mathrm{Pt}\left(\mathrm{NH}_{3}\right)\right] \mathrm{Cl}_{2}\right)$ for about 48 hours and then soaked in $0.1 \%$ sodium borohydride $\left(\mathrm{NaBH}_{4}\right)$ for about 3 hours. 
Similar methods of plating platinum onto Nafion ${ }^{\mathrm{TM}}$ membranes have been reported by a number of researchers $(50 ; 1 ; 33 ; 32 ; 29 ; 46 ; 25 ; 52 ; 40)$. Using this process, platinum electrodes that had a measured surface resistance of about $6 \Omega / \mathrm{cm}$ were formed on the Nafion ${ }^{\mathrm{TM}}$ membrane. This measurement of the surface resistance is higher than typical measured values for copper electrodes prepared in a similar way $(\sim 3 \Omega / \mathrm{cm})$. This can be explained by the fact that platinum has a bulk electrical resistivity more than six times that of copper. It is interesting to note that whereas the copper reduction process occurs very rapidly in sodium borohydride solution of similar concentration, the reduction of platinum is much slower and no deposit is visible in the membrane until 30 minutes or so after the membrane has been placed in the reducing agent solution. Once processes for depositing good quality platinum and copper electrodes onto Nafion membranes were developed, the transition to depositing electrodes composed of platinum/copper alloys was easliy realized.

\subsection{Details of New Process}

Based on the initial results with using electrodes composed of noble and non-noble metals and alloys of noble and non-noble metals, further investigation was performed to study the stability of electrodes composed of a copper/platinum alloy and the performance of the actuators made using these electrodes. The first step in this investigation was to determine a way to control the relative proportions of copper and platinum in the electrodes. Yeager et al. have shown that the relative ionic fraction of different ions in a Nafion ${ }^{\mathrm{TM}}$ membrane can be controlled by controlling the relative ionic fraction of the ions in the exchanging solution $(65 ; 54 ; 63)$. For ions whos selectivity coefficients are known, the proper ionic fraction in the solution can be determined based on the desired ionic fraction in the membrane. For the copper/platinum electrodes plated in the current study, tetrammine platinum chloride $\left(\left[\mathrm{Pt}\left(\mathrm{NH}_{3}\right)\right] \mathrm{Cl}_{2}\right)$ was used to source the platinum ions and cupric sulfate $\left(\mathrm{CuSO}_{4}\right)$ was used to source the copper ions. Unfortunately, the selectivity coefficients for the exchange of the copper and tetraammine platinum ions with hydrogen ion is not known, and therefore the proper proper proportion of ions to be mixed in the exchanging solution must be determined by trial-and-error. This is as much the case because the proportion of copper to platinum in the electrode that will eliminate the problem of electrode oxidation is also not known.

The goal of this study was to minimize the amount of platinum present in the 
electrode while eliminating the problem of electrode oxidation. It is advantageous to have less platinum in the electrode for several reasons. First, platinum is very expensive; the purpose of this research project from the beginning was to reduce the cost of ionic polymer transducers by reducing the cost of the electrodes used to make them. Second, platinum has a density that is 2.36 times greater than the density of copper, meaning that electrodes that contain large amounts of platinum will be heavier as a result, increasing the overall mass of the ionic polymer device and decreasing its mass energy density. And third, the electrical resistivity of platinum is over six times that of copper, which will adversely affect the surface conductivity if large amounts of platinum are used.

In the current embodiment of the impregnation/reduction process for plating metal electrodes onto Nafion ${ }^{\mathrm{TM}}$ membranes, copper and platinum ions are exchanged into the membrane simultaneously and then reduced simultaneously at the membrane surface in aqueous solution of sodium borohydride. This process produces an electrode composed of an alloy of copper and platinum. In order to determine the amount of platinum necessary to stabilize the electrodes, several samples were made and tested, with different relative proportions of copper to platinum. As before, longevity testing was used to determine the stability of the electrodes under actuation conditions. Because there are a large number of parameters whose effect on the formation and stability of the metal electrodes is unknown, many of them were held constant during this testing, including the soak time in the exchanging solution, the soak time in the reducing solution, the temperature of the solutions, the pretreatment of the membrane, and the amplitude, frequency, and waveform of the applied actuation input.

Throughout this study, the molarities of the copper and platinum compounds in the exchanging solution were changed, but the total molarity of these two compounds was maintained at $0.01 \mathrm{M}$. The membrane samples were pretreated in the same manner for each test, first by roughening on both sides with 600 grit sandpaper, then by ultrasonic cleaning, and finally by boiling for 30 minutes in $0.5 \mathrm{M} \mathrm{HCl}$. The soak in the exchanging solution was performed at room temperature for 30 minutes, with agitation by a magnetic stir bar. The soak in the reducing agent solution was performed at room temperature for two hours, again with agitation by a magnetic stir bar. Initially, a fixture was used to tension the membrane (see Figure 3.13), but the quality of the electrodes made using this fixture were poor, presumably because its large size prevented sufficient mixing in the 
exchanging solution. Following this result, the samples were held by clamping one edge vertically between two glass stirring rods, held together by rubber bands. The unclamped portion of the sample was then allowed to swing freely in the solution, like a flag.

The first sample made using this process was fabricated by soaking in an exchanging solution composed of $50 \%$ copper ions and $50 \%$ platinum ions; this solution contained 0.005 $\mathrm{M}$ cupric sulfate and $0.005 \mathrm{M}$ tetrammine platinum chloride. Following ion-exchange in this solution for 30 minutes, the sample was rinsed with de-ionized water and absorbed metal ions were reduced at the surface in $0.1 \%$ (wt.) sodium borohydride solution for one hour. The presence of copper in the electrode is evident when the sample is placed in the reducing agent solution, as a color change in the membrane is immediatey observed upon immersion in this solution. Past experience indicates that when the exchanging solution is composed only of platinum, no color change will be immediately visible when the reduction is initiated. By contrast, the copper reduction occurs very rapidly and a color change in the membrane is evident immediately upon initiation of reduction. Following this initial color change, a deposit of platinum could slowly be observed forming on the membrane, and by the end of the reduction process, the appearance of the sample looked no different that that of a sample plated with only platinum. The measured surface resistance of the electrodes following all processing was approximately $10 \Omega / \mathrm{cm}$. Images of the of the cross-section of this sample were collected using scanning elecron microscopy-see Figure 4.3. As can be seen in the figure, the thickness of the electrodes appears to be less than one micron. The composition of the electrode was also determined, by using X-ray analysis as described in Chapter 2. From this testing, the electrode was found to consist of $99 \%$ (atomic) platinum, with only very small amounts of copper present. Based on the extremely small amount of copper found in these electrodes, it seems that the Nafion ${ }^{\mathrm{TM}}$ membrane has a preference for the tetraammine platinum ion over the copper ion. Further testing investigated the properties of copper/platinum electrodes made using exchanging solutions containing lower relative proportions of tetraammine platinum ion.

Following this initial result, another sample of Nafion ${ }^{\mathrm{TM}}$ was plated with a copper/platinum alloy, this time by soaking in a solution of $0.008 \mathrm{M}$ cupric sulfate and $0.002 \mathrm{M}$ tetrammine platinum chloride for 30 minutes. The sample was then rinsed with de-ionized water and soaked in a solution of $0.1 \%$ (wt.) sodium borohydride for two hours. Again, an initial deposition of copper was formed in the membrane, indicated by a rapid color change. 


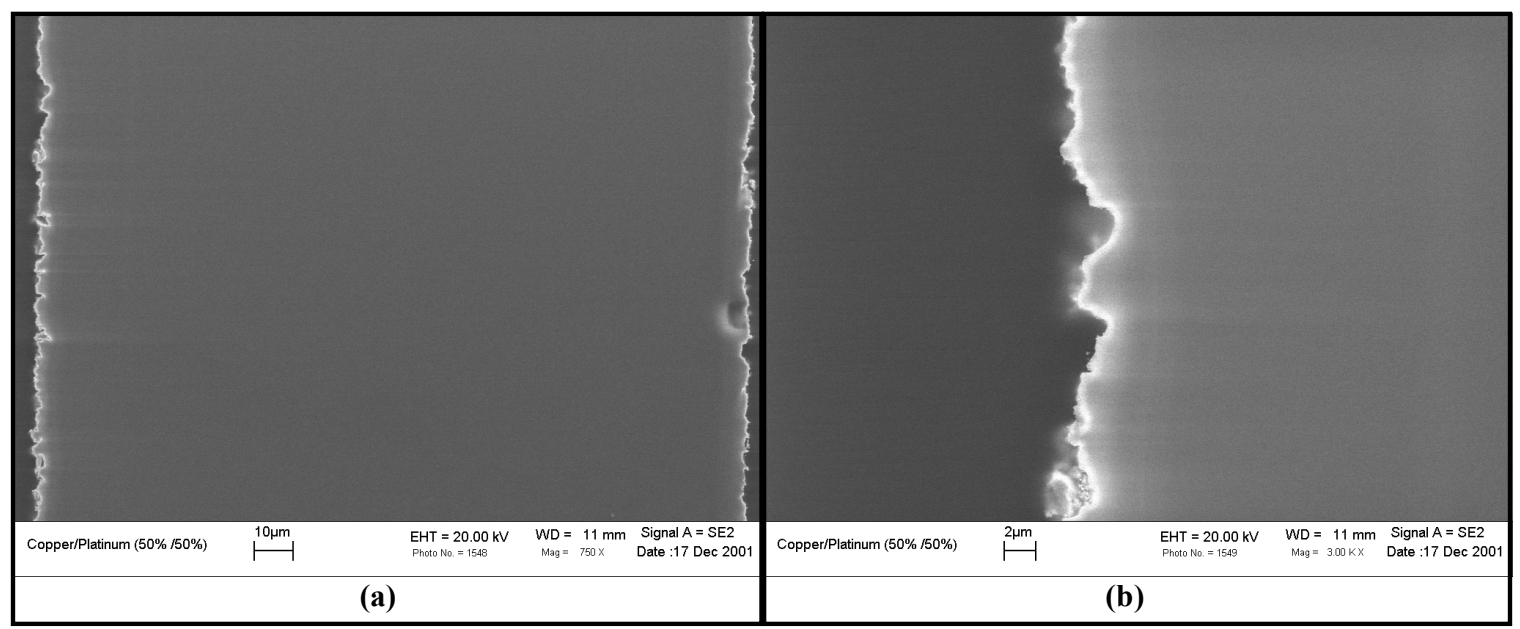

Figure 4.3: (a) Scanning electron micrographs of the cross-section of a sample plated using a 50\% copper, $50 \%$ platinum plating solution. (b) Close-up of the left electrode.

This was followed by a slow deposition of platinum and again, the final appearance of the electrodes was identical to a sample plated with only platinum. The measured surface resistance of this sample was about $10 \Omega / \mathrm{cm}$. Again, scanning electron microscopy was used to image the cross-section of this sample - see Figure 4.4. From this figure it is evident that the electrode is much thicker and has a visibly different structure than the electrode made using the 50\% copper, 50\% platinum solution. Again, X-ray analysis was performed; the the composition of the electrode was found to be about $90 \%$ (atomic) platinum and about $10 \%$ (atomic) copper. This result is the average composition across several scans of different areas of the electrode. Longevity testing of this sample was also performed, the results of which are summarized in Figure 4.5 for several samples. As can be seen in the figure, of the two samples tested, one retained $92 \%$ of its initial motion after almost a quarter of a million actuation cycles at $1.25 \mathrm{~V}$, the other retained $56 \%$ of its initial motion. The reason for the large discrepancy in the stabilty of the two samples is unknown, but may be attributed to inconsistencies in the copper/platinum coating.

The results of this test confirmed that the relative proportions of copper and platinum in the electrode can be controlled by changing the relative proportions of copper and platinum ions in the exchanging solution. Based on this result, more samples were fabricated in which the loading of platinum was reduced further. Each of these samples was processed in the same way, except that the composition of the cupric sulfate/tetraammine platinum chloride solution was changed. The results of longevity testing performed with 


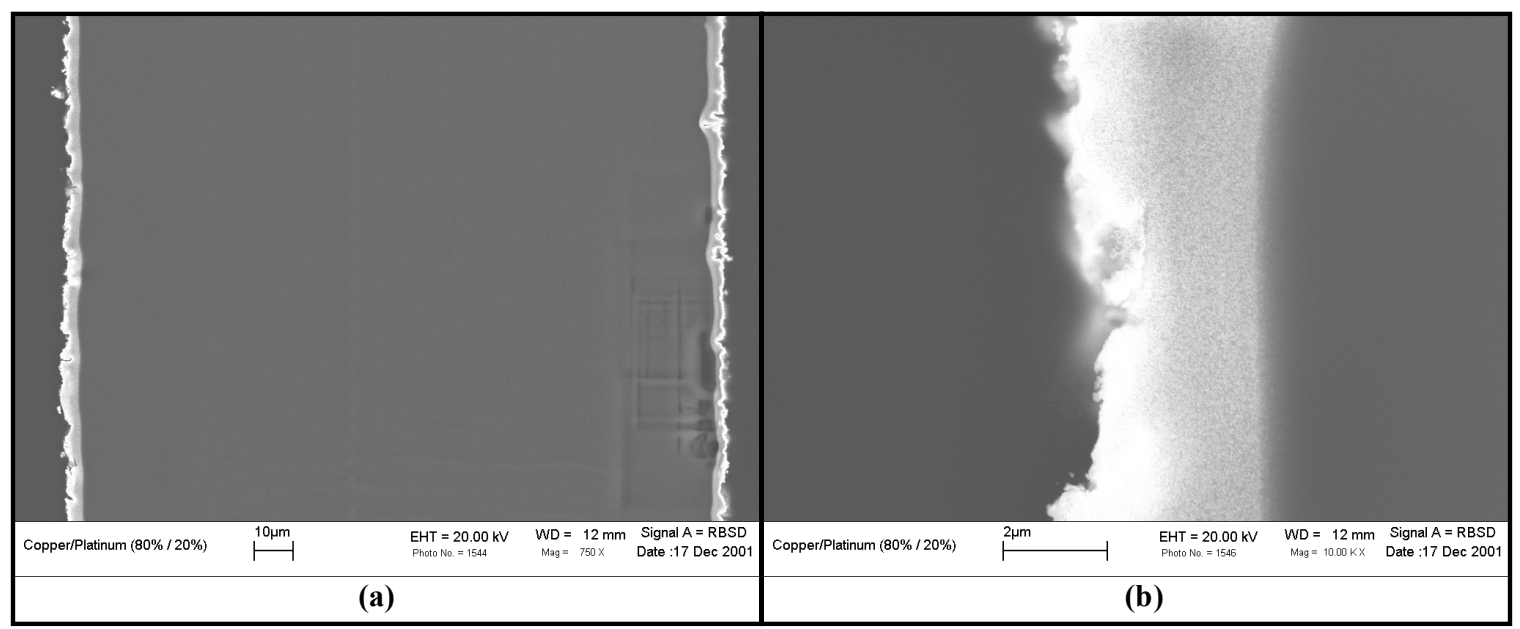

Figure 4.4: (a) Scanning electron micrographs of the cross-section of a sample plated using a $80 \%$ copper, $20 \%$ platinum plating solution. Note that the damage near the right edge was caused by the X-ray source. (b) Close-up of the left electrode.

these samples can be found in Figure 4.5. As can be seen in the figure, as the amount of platinum present in the exchanging solution is decreased, the stability of the electrode is decreased. Scanning electron micrographs of these samples were also obtained, and Xray analysis was performed to determine the composition of metals in the electrode. This testing revealed that as the relative proportion of platinum in the exchanging solution was decreased, the amount of platinum present in the electrode also decreased-see Figure 4.6. This was also witnessed by the difference in the appearance of the electrodes both during and after the reduction process of samples processed in exchanging solutions containing different amounts of platinum. For samples fabricated using exchanging solutions with more than $90 \%$ relative proportion of copper ion, a shiny copper coating is observed forming at the surface of the membrane, before being obscured by a deposition of platinum. When the relative proportion of copper ion is lower than $90 \%$, a light brown copper deposit is formed below the surface of the membrane, but a shiny copper layer is not evident. Again, this copper deposit is gradually obscured by a deposition of platinum. Also, for samples fabricated using solutions very rich in copper ion, the final appearance of the electrode is a pink/grey color, indicating that both copper and platinum metal phases exist near the surface of the electrode. SEM and X-ray analysis confirmed that these two metals also exist within the polymer membrane, to depths of 0.5 to 7 microns. See Figure 4.7 for images of the crosssections of a sample plated using an exchanging solution containing $90 \%$ copper and $10 \%$ 
platinum. From the figure, it is evident that these electrodes have a fundamentally different morphology than the electrodes plated using solutions containing relatively less copper. These electrodes exhibit a diffuse layer of metal about 3-4 microns deep as well as dendritic formations that extent about 6-7 microns into the polymer. This structure is similar in appearance to electrodes made by Oguro et al. using a similar impregnation/reduction process to plate gold electrodes onto Nafion ${ }^{\mathrm{TM}}$ membranes $(44 ; 41)$.

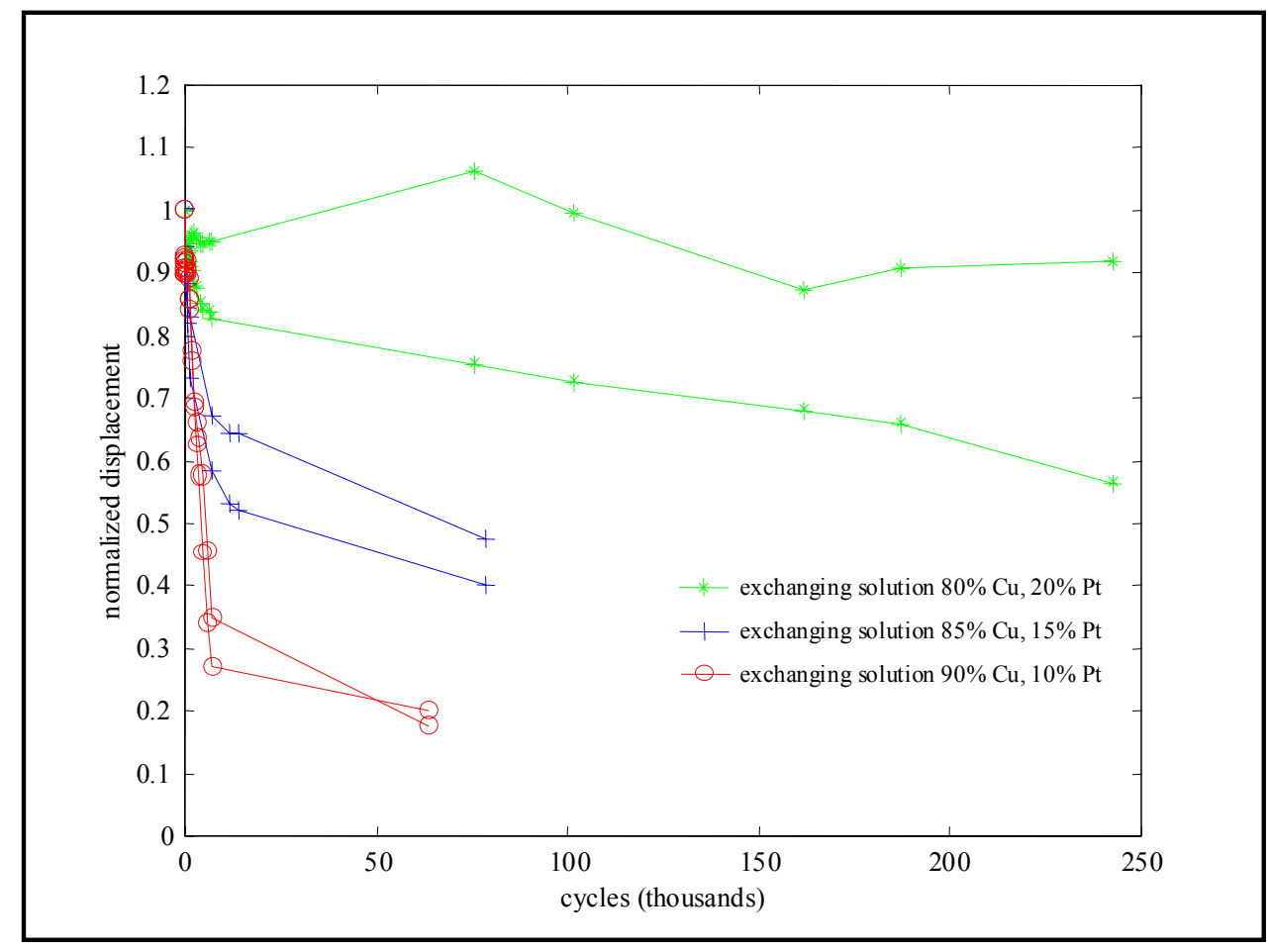

Figure 4.5: Normalized tip displacement versus the number of actuation cycles for three sets of two samples each plated with a copper/platinum alloy electrode using different ion-exchange solutions. The input was a $1.25 \mathrm{~V}, 1.0 \mathrm{~Hz}$ sine wave.

The testing performed with exchanging solutions of different compositions has confirmed that the stability of the copper/platinum alloy electrodes is a function of the relative proportion of platinum in the electrode. An interesting result is the relationship between the relative proportions of the ions in the exchanging solution and the relative proportions of the metals in the electrode - see Figure 4.6. This data seems to indicate that the membrane has a much higher preference for the tetraammine platinum ion that for the copper ion. Based on the work of Yeager et al., a plausible explanation for this phenomenon may be that the copper ion is more solvated, and thus the platinum ion is preferred. Prior to the ion-exchange process, the membrane is put into the hydrogen form by boiling it in 0.5 


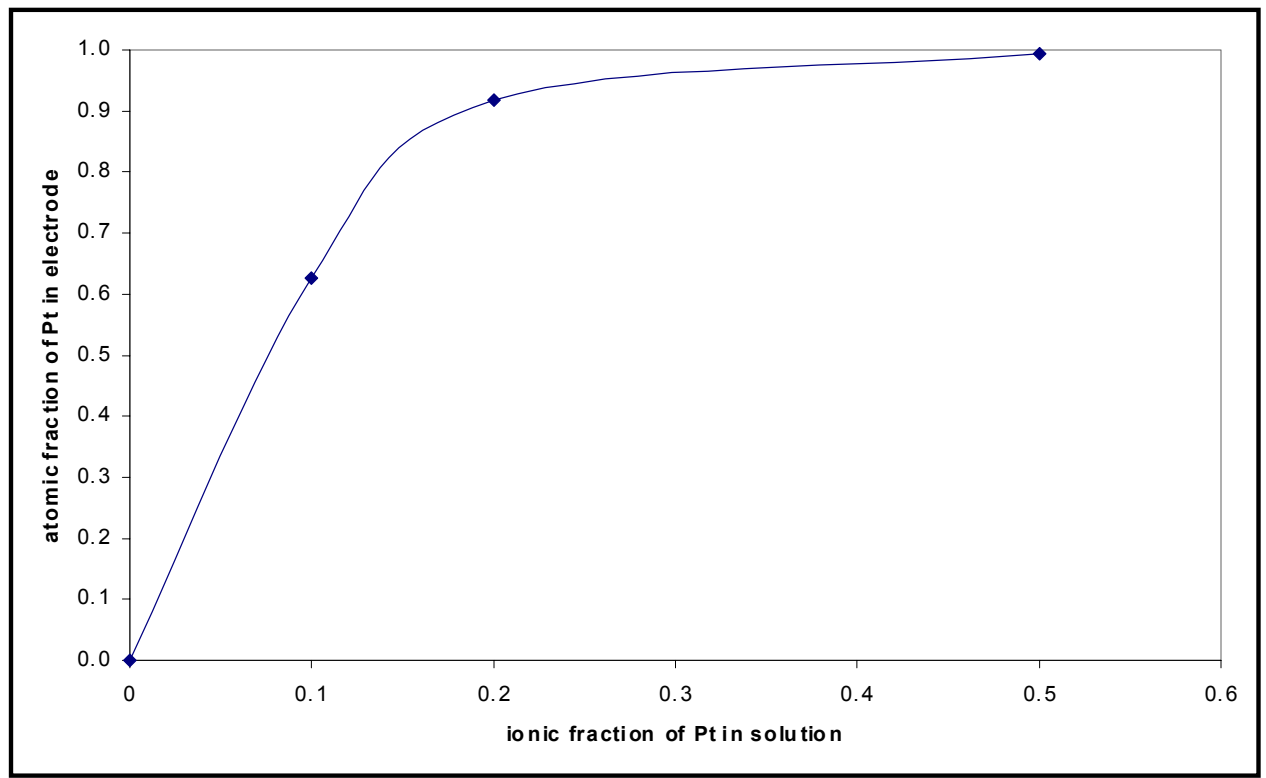

Figure 4.6: Measured atomic fraction of platinum metal in electrodes versus ionic fraction of tetraammine platinum ion in exchanging solution. This data was obtained by averaging the results of several X-ray scans for each sample.

$\mathrm{M} \mathrm{HCl}$. Because the hydrogen ion is highly solvated, this is the most hydrated state that the polymer can acheive. When another ion replaces a hydrogen ion, some water is released into the outer solution and the polymer relaxes; both of these processes produce entropy, which drives the exchange reaction toward completion. If two ions are present in the outer solution, the polymer will prefer the one that is less solvated, because exchange with this ion will generate the largest possible loss of water by the polymer. This is a very simplistic view of the ion-exchange process, electrostatic forces may also play a role. However, both the copper and the tetraammine platinum ion are of charge +2 , so there should be very little difference in the electrostatic forces between these ions and the sulfonate exchange sites. Therefore, the ion that is less solvated should be preferred by the polymer. Because the copper ion is smaller than the tetraammine platinum ion, it has a higher charge density, and therefore it is likely more solvated. So experiment follows theory for this case; the ion that is presumably less solvated is preferred by the membrane and the composition of the exchanging solution is adjusted accordingly.

Another interesting phenomenon is observed when the composition of the exchanging solution is held constant and the soak time in the exchanging solution is changed. For all of the experiments in which the composition of the copper/platinum solution was changed, 


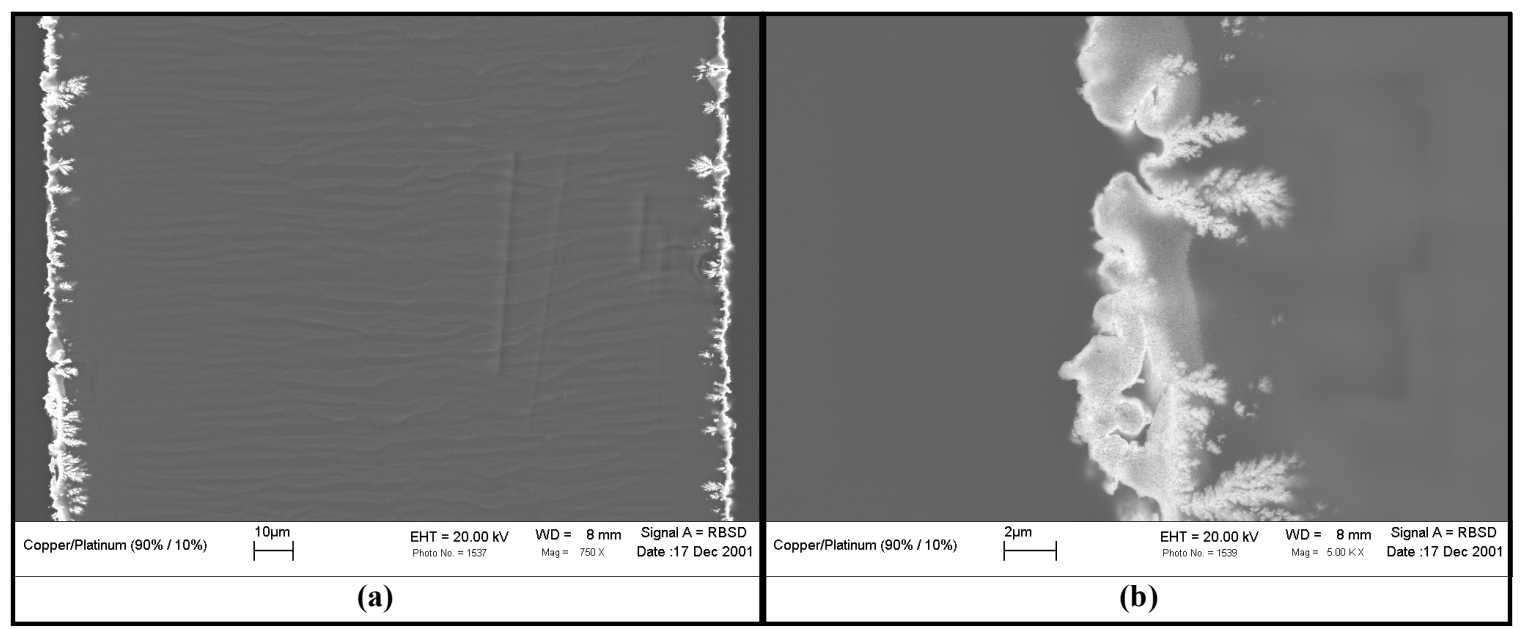

Figure 4.7: (a) Scanning electron micrographs of the cross-section of a sample plated using a $90 \%$ copper, $10 \%$ platinum plating solution. (b) Close-up of the left electrode.

the soak time was held constant at 30 minutes, with agitation. When this soak time was varied, interesting differences were observed in the resulting electrodes, however. In the first experiment, a sample of pretreated Nafion ${ }^{\mathrm{TM}}$ membrane was soaked in a solution of 0.009 $\mathrm{M} \mathrm{CuSO}_{4}$ and $0.001 \mathrm{M}\left[\mathrm{Pt}\left(\mathrm{NH}_{3}\right)\right] \mathrm{Cl}_{2}$ overnight. This sample was then reduced in a solution of $0.1 \%$ (wt.) $\mathrm{NaBH}_{4}$ for two hours. During and after the exchange process, the electrode appeared to be mostly platinum. The measured surface resistance of the electrodes was 10$12 \Omega / \mathrm{cm}$. Another experiment was performed in which the same copper/platinum solution was used, but the soak time was reduced to 15 minutes. Again, the reduction was done in $0.1 \%$ (wt.) $\mathrm{NaBH}_{4}$ for two hours. The measured surface resistance was between 6 and $11 \Omega / \mathrm{cm}$ and the electrode visually appeared to be composed of mostly copper, with only small amounts of platinum. While a visual observation is not definitive evidence that the relative proportions of copper and platinum were different in these two samples, it does seem logical that a sample that looks more like platinum contains more platinum than a sample that looks more like copper. Based on this result, a reasonable conclusion may be that the diffusion coefficient for the copper ion in the Nafion ${ }^{\mathrm{TM}}$ polymer is higher than the diffusion coefficient for the tetraammine platinum ion in the Nafion ${ }^{\mathrm{TM}}$ polymer, therefore a longer soak time will increase the presence of platinum in the electrodes. A simple explanation for this may be that the copper ion diffuses through the polymer faster because it is smaller, therefore it has less physical interaction with the polymer matrix as it moves through the membrane. Yeager has published similar results for sodium and cesium ions (both of charge 
+1 ) exchanging with Nafion ${ }^{\mathrm{TM}}$ membranes initially in the hydrogen form (63). Although the selectivity coefficient for cesium to hydrogen is 7.5 times greater than that for sodium to hydrogen, the self-diffusion coefficient for sodium is 18.1 times greater than the self-diffusion coefficient for cesium. This result fits the current argument, as the cesium ion is larger (and therefore presumably less solvated) than the sodium ion.

The initial results with the copper/platinum co-reduction processes indicates that the relative amounts of copper and platinum in the electrode can be controlled, and that changing the relative amounts of copper and platinum will affect the stability of these electrodes. In the current embodiment of this process, an exchanging solution containing $95 \%$ copper ion and $5 \%$ platinum ion is used to impregnate copper and tetraammine platinum ions into the membrane simultaneously. These ions are then reduced in an aqueous solution of sodium borohydride to form metallic electrodes. The thickness of these electrodes is approximately 2 microns - see Figure 4.8 for SEM images. X-ray analysis of these electrodes to determine their composition has not been performed at this time. By itself, this electrode is not stable. This is not surprising considering the longevity results presented in Figure 4.5. From this figure, it is evident that even electrodes containing more platinum than those produced using this process are not indefinitely stable under actuation conditions. The stabililty of this copper/platinum alloy electrode may be improved by plating it with a thin layer of gold, however. Using the same electroless plating process described in Chapter 3 , a thin layer of gold is plated over the copper/platinum alloy to help protect the electrode from oxidation. Refer to Figure 4.9 for photos of Nafion ${ }^{\mathrm{TM}}$ membranes processed using this technique both before and after the deposition of the gold layer. This gold layer can be seen in Figure 4.10; from this image it is apparent that the gold layer is about 50-80 nanometers thick. Using this process copper/platinum alloy electrodes with gold overlayers have been fabricated and a sample plated in this way has been actuated at $1.25 \mathrm{~V}$ for over 150,000 cycles with only an $8 \%$ drop in the free tip deflection-see Figure 4.11. Having confirmed that ionic polymer transducers could be reliably manufactured using an exchanging solution of $95 \%$ copper ion and $5 \%$ platinum ion with subsequent electroless gold plating, further testing of devices made using this process was performed in order to quantify their electrical and mechanical properties. 


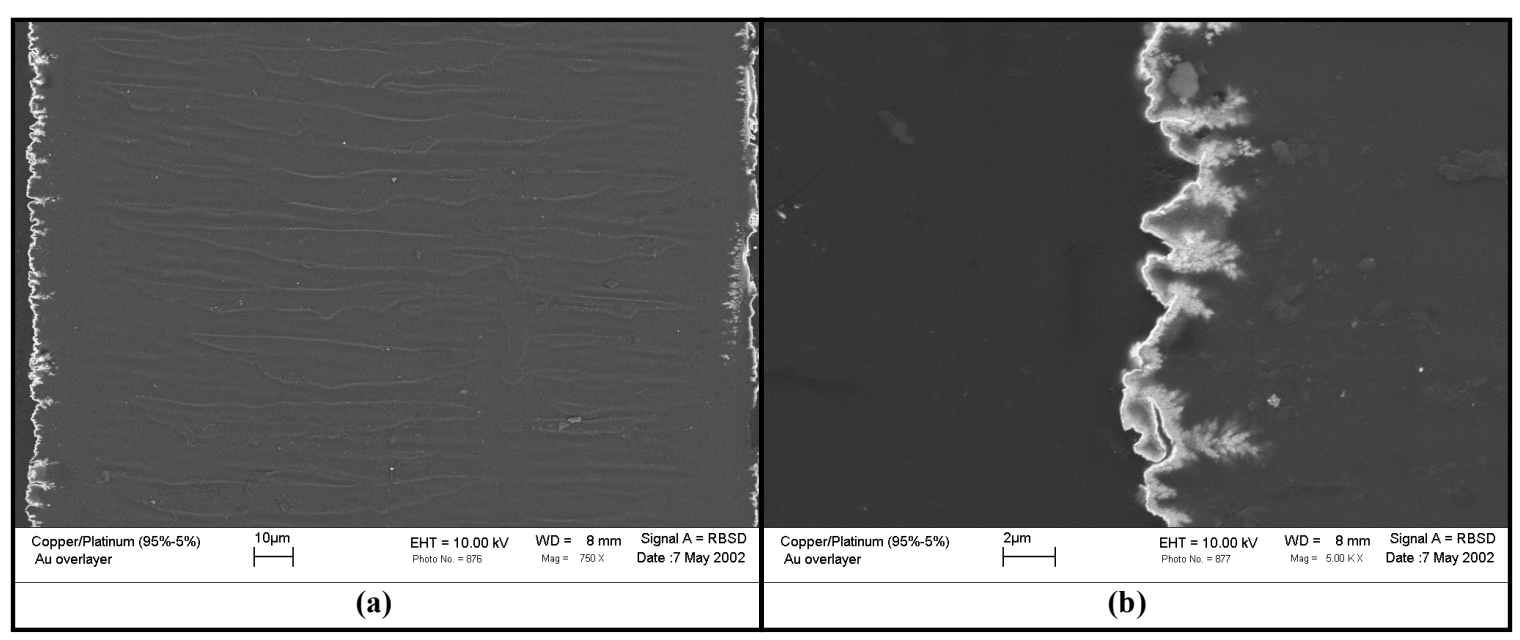

Figure 4.8: (a) Scanning electron micrographs of the cross-section of a sample plated using a $95 \%$ copper, $5 \%$ platinum plating solution. (b) Close-up of the left electrode. Note that the gold overlayer is visible in these images.

\subsection{Characterization of New Process}

Once a process for making ionic polymer transducers using non-precious metal electrodes had been developed and tested, further testing was performed in order to evaluate the mechanical and electrical properties of these devices. Of partucular interest are measurements of the sensing coefficient, density, energy output, modulus of elasticity, and longevity. These metrics are useful for comparing these new ionic polymer actuators to the baseline material kindly supplied by Dr. Shahinpoor of the University of New Mexico, as well as for making comparisons to other types of smart material technologies.

Newbury and Leo have reported on the sensing capabilities and energy densities of the baseline material, which is composed of Nafion ${ }^{\mathrm{TM}}-117$ polymer membrane with gold electrodes. In their tests, Newbury and Leo have found the sensing coefficient of this material to be about $1.0 \frac{\mu \mathrm{A}}{\mathrm{mm} / \mathrm{s}}$ for a sample $5 \mathrm{~mm}$ wide with a $17 \mathrm{~mm}$ free length $(37 ; 36)$. This coefficient was determined by clamping the sample in a cantilever configuration and applying a quasi-step change in the displacement at the tip of the sample while the velocity of the tip and the short-circuit current through the sample were recorded. Further experiments using the frequency response method described in Chapter 2 have shown this value to be on the order of $0.2-0.8 \frac{\mu \mathrm{A}}{\mathrm{mm} / \mathrm{s}}$ for $5 \mathrm{~mm}$ by $15 \mathrm{~mm}$ samples. They have also measured the blocked force and free deflection of these cantilever benders in order to determine the useful energy density of these devices. In these tests, a sample $5 \mathrm{~mm}$ wide was clamped in a 


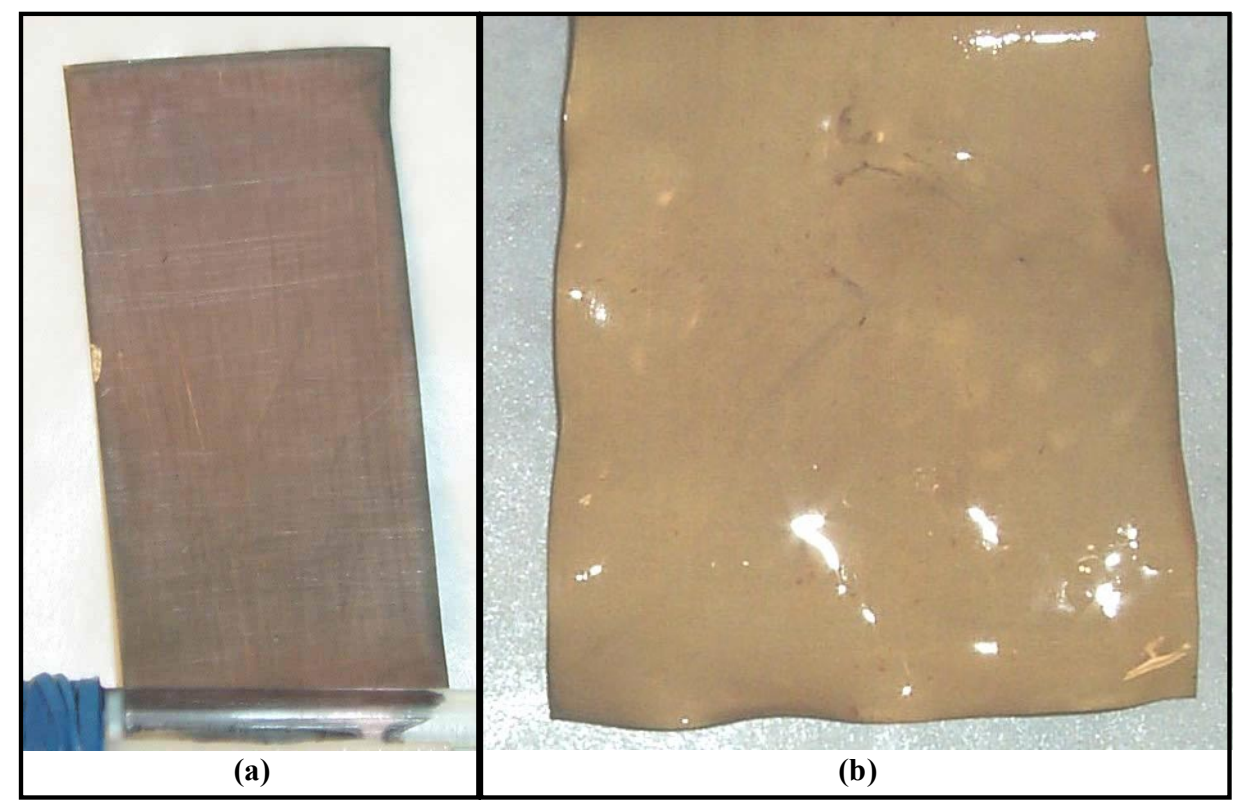

Figure 4.9: Photos of samples of Nafion ${ }^{\mathrm{TM}}$ processed using the new method (a) before deposition of the gold layer, (b) after deposition of the gold layer.

cantilevered configuration such that its free length was $17 \mathrm{~mm}$. A step voltage was then applied across the electrodes of the device and the force and displacement at the tip was recorded. This experiment was performed several times, with the amount that the polymer deflected before contacting the load cell varied from one test to the next. By this method, a plot of the generated tip force versus the allowed deflection was formed. It should be noted that these polymers experience a large initial deflection followed by a slow decay and that these force/deflection curves represent peak, not steady-state, values. Newbury and Leo discovered several important properties of these ionic polymer benders that will now be discussed. First, the peak force/deflection curves are linear. That is, the amount of peak force generated at the tip of a bender when a voltage is applied is a linear function of the amount that the bender deflects from its neutral position. Second, the amount of force and deflection generated depends on the initial curvature of the sample. The samples supplied by Dr. Shahinpoor had an initial curvature even with no voltage or external force applied. When this initial curvature was in the same direction as the induced motion caused by the actuation voltage, a blocked force of $0.67 \mathrm{mN}$, a free deflection of $0.51 \mathrm{~mm}$, and a corresponding energy density of $3.10 \mathrm{~mJ} / \mathrm{kg}$ was measured, for a step input of $1.25 \mathrm{~V}$. When the initial curvature opposed the induced motion, a blocked force of $1.63 \mathrm{mN}$, a free deflection of $0.78 \mathrm{~mm}$, and a corresponding energy density of $11.52 \mathrm{~mJ} / \mathrm{kg}$ was measured. 


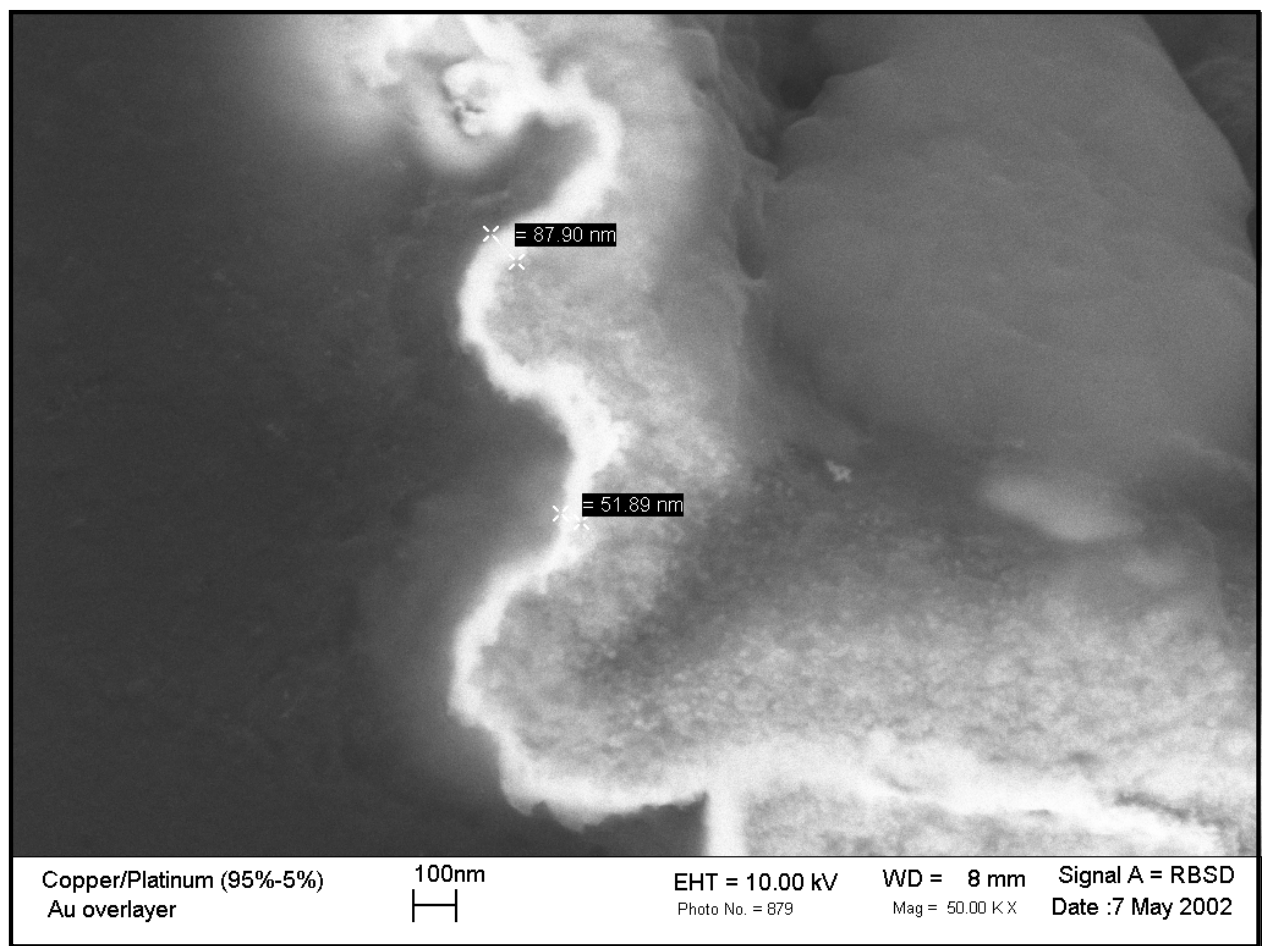

Figure 4.10: Scanning electron micrograph showing a close-up of the gold overlayer on a sample plated with a copper/platinum alloy and subsequently with gold. Note that the gold layer is approximately 50-80 nanometers thick.

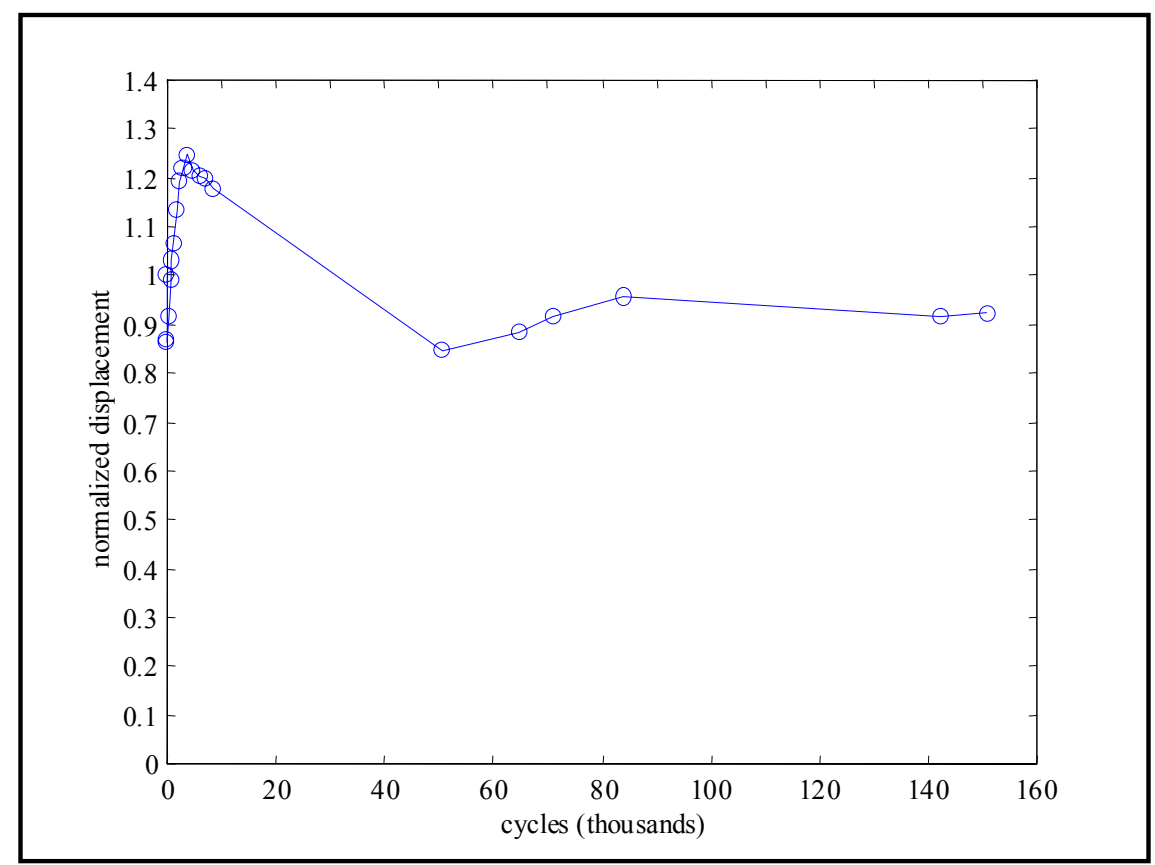

Figure 4.11: Normalized tip displacement versus the number of actuation cycles for a sample plated using an exchanging solution of $95 \%$ copper ion and $5 \%$ tetraammine platinum ion with a protective gold layer. The input was a 1.25 $\mathrm{V}, 1.0 \mathrm{~Hz}$ sine wave. 
Table 4.1: Sensitivity coefficients of $5 \mathrm{~mm}$ wide strips of $\mathrm{Nafion}^{\mathrm{TM}}-117$ and Nafion $^{\text {TM }}$-115 plated using the new process and clamped in a cantilevered configuration with a $17 \mathrm{~mm}$ free length. All data are in $\frac{\mu \mathrm{A}}{\mathrm{mm} / \mathrm{s}}$.

\begin{tabular}{|r|r|}
\hline Nafion $^{\mathrm{TM}}-117$ & Nafion $^{\mathrm{TM}}-115$ \\
\hline 0.0757 & 0.0672 \\
\hline 0.0917 & 0.0791 \\
\hline 0.1075 & 0.0823 \\
\hline 0.0889 & 0.0895 \\
\hline
\end{tabular}

In order to compare the ionic polymer devices fabricated using the current method to those supplied by Dr. Shahinpoor, similar testing of the sensing capability and energy density was performed. In the current work, the sensing coefficient is measured using the procedure described in Chapter 2, in which the sample is clamped in a cantilever configuration and the tip is deflected using an electromagnatic shaker. During this test the short-circuit current is recorded as a function of input frequency for a range of frequencies from 1 to $20 \mathrm{~Hz}$. This test demonstrated that these polymers exhibit a nearly flat frequency response with a steady loss of phase over this range of frequencies - see Figure 4.12. The sensitivity coefficient of these samples is then taken as the average of the measured sensitivities over the range of input frequencies. Please refer to Table 4.1 for the sensitivity coefficients of several samples of Nafion ${ }^{\mathrm{TM}}-117$ (nominal thickness 180 microns) and Nafion ${ }^{\mathrm{TM}}-115$ (nominal thickness 125 microns) that have been plated using the new method - these samples are $5 \mathrm{~mm}$ wide with a $17 \mathrm{~mm}$ free length. Note that the average of these coefficients is $0.0910 \frac{\mu \mathrm{A}}{\mathrm{mm} / \mathrm{s}}$ for the samples of Nafion ${ }^{\mathrm{TM}}-117$ and $0.0795 \frac{\mu \mathrm{A}}{\mathrm{mm} / \mathrm{s}}$ for the samples of Nafion ${ }^{\mathrm{TM}}-115$. The reason that the sensitivity coefficient is smaller for the samples of Nafion ${ }^{\mathrm{TM}}-115$ is that these samples have a smaller thickness than the samples of Nafion ${ }^{\mathrm{TM}}-117$, therefore the rate of induced bending strain for a given velocity input is smaller. Also, it is interesting to note that the measured sensitivity coefficient for the samples of Nafion ${ }^{\mathrm{TM}}-117$ is about a factor of ten less than the measured sensitivity coefficient for the baseline material. This may be due to the differences in the way that the sensing test was performed between the two materials. Another explanation may be that differences in the other properties of these materials, which will be discussed, play a role.

Another important property of the Nafion ${ }^{\mathrm{TM}}$ membranes plated using the new method is their mass density. The mass density of these transducers is useful for determining the energy density and comparing these devices to others. The density was measured 


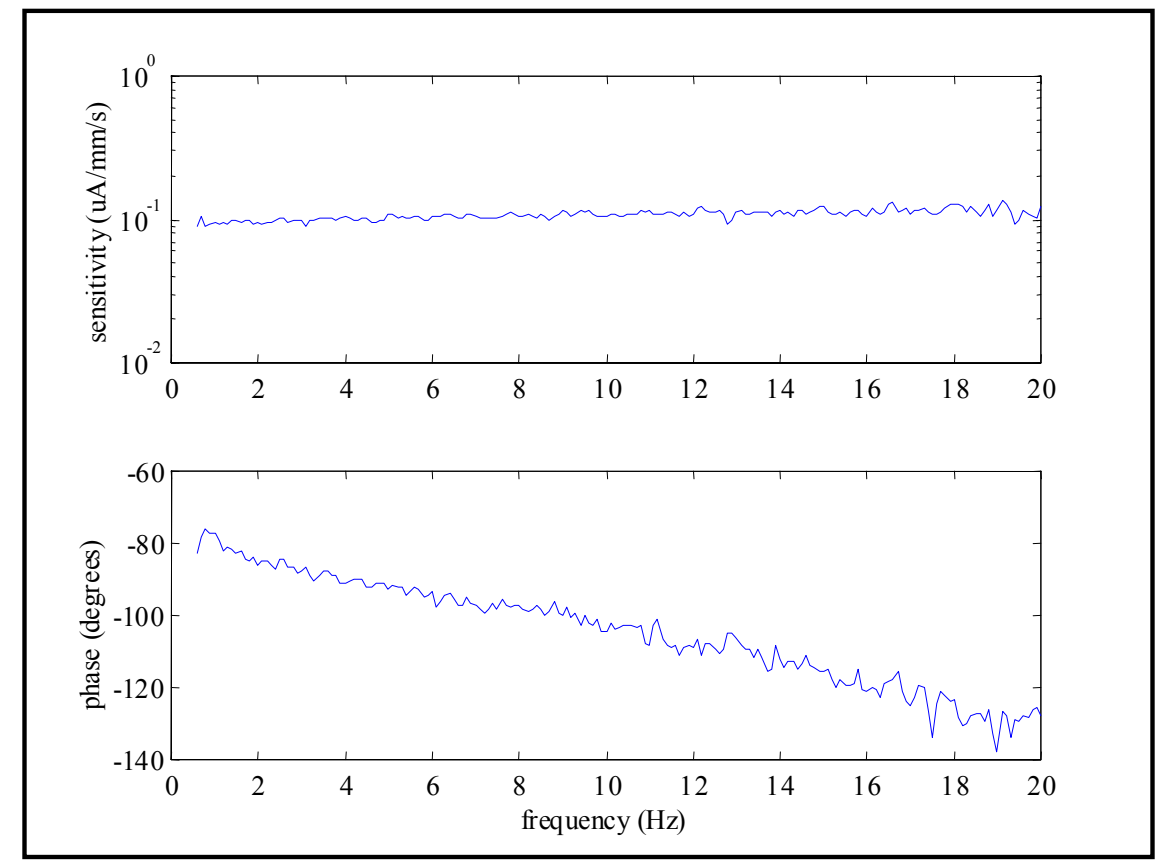

Figure 4.12: Sensing response versus frequency of a sample of Nafion ${ }^{\mathrm{TM}}-117$ plated with a copper/platinum alloy and a gold overlayer. The average sensitivity over the indicated frequency range is $0.1075 \frac{\mu \mathrm{A}}{\mathrm{mm} / \mathrm{s}}$.

using the procedure outlined in Chapter 2, in which individual samples were weighed to $0.0001 \mathrm{gm}$ using an electronic analytical balance and their size measured using a steel scale graduated in millimeters. The membranes were weighed in their hydrated state, although their surfaces were dried prior to weighing. Also, the membrane samples were in the lithium ion form when these measurements were taken. The volume of each sample was calculated by multiplying its width, length, and thickness, where the thickness used was the nominal thickness of the dry Nafion ${ }^{\mathrm{TM}}$ membrane (180 microns for Nafion ${ }^{\mathrm{TM}}-117,125$ microns for Nafion ${ }^{\mathrm{TM}}-115$ ). Please refer to Table 4.2 for the measured densities of several samples of Nafion $^{\mathrm{TM}}-117$ and Nafion ${ }^{\mathrm{TM}}-115$ plated using the current method. The average density of the samples of Nafion ${ }^{\mathrm{TM}}-117$ is $2034.7 \mathrm{~kg} / \mathrm{m}^{3}$ and the average density of the samples of Nafion ${ }^{\mathrm{TM}_{-}}-115$ is $2158.4 \mathrm{~kg} / \mathrm{m}^{3}$. Please note that this test is not well controlled, and this difference could easily be attributed to experimental error, but another explanation may exist. The electrodes in the two plated samples are likely to be approximately the same thickness and composition, since the two samples were processed in the same way. By this argument, the electrodes in the sample of Nafion ${ }^{\mathrm{TM}}-115$ would constitute a larger percentage of the total mass than the electrodes in the sample of Nafion ${ }^{\mathrm{TM}}-117$. Because 
Table 4.2: Measured densities of several strips of Nafion ${ }^{\mathrm{TM}}-117$ and Nafion $^{\mathrm{TM}}$ 115 plated using the current method. All data are in $\mathrm{kg} / \mathrm{m}^{3}$.

\begin{tabular}{|r|r|}
\hline Nafion $^{\text {TM }}-117$ & Nafion $^{\text {TM}}-115$ \\
\hline 2231.1 & 2056.2 \\
\hline 1840.0 & 2220.4 \\
\hline 2031.1 & 2233.7 \\
\hline 2084.4 & 2208.0 \\
\hline 1986.7 & 2073.6 \\
\hline
\end{tabular}

the densities of the metals in the electrodes are higher than the density of the Nafion ${ }^{\mathrm{TM}}$, the situation described would predict that as the thickness of the Nafion ${ }^{\mathrm{TM}}$ membrane is decreased, the overall density of the plated device is increased.

In their work with the baseline material, Newbury and Leo found the density of this material to be approximately $3200 \mathrm{~kg} / \mathrm{m}^{3}$ using the same method as described in Chapter 2. Therefore, although the new materials generate less output energy than the baseline material, the energy density of the new material is at least as high as that of the baseline material because the mass density of the new material is so much lower. The reason that the density of the baseline material is over $50 \%$ higher than the materials made using the new process is that the baseline material contains more metal than the new materials. This is evident by comparing the thickness of the electrodes. While the electrodes on the baseline material are 4-15 microns thick, the electrodes on the new materials are only about 2 microns thick. Also contributing to its higher density is the presence of isolated gold particles throughout the baseline material. Although the thicker electrodes of the baseline material may offer improved charge transfer to the device, the larger mass of these electrodes adversely affects the energy density and bandwidth of these devices. The optimal electrode will be the one that results in the the lowest mass and the largest energy output of the actuator.

Once the density of the plated Nafion ${ }^{\mathrm{TM}}$ membranes is known, the mass of the active part of a cantilevered bender made of this material can be inferred from its volume. This allows for measurements of peak blocked force and peak free deflection to be directly converted into mass energy densities. As described in Chapter 2, the blocked force and free deflection are measured with the polymer samples clamped in a cantilevered configuration.

$$
e_{m}=\frac{F_{\text {blocked }} * \delta_{\text {free }} * V_{\text {active }}}{2 * \rho}
$$


Table 4.3: Measured peak blocked force, peak free deflection, and resulting peak energy densities of five strips of Nafion ${ }^{\mathrm{TM}}-117$ plated using the new process. Each strip was $5 \mathrm{~mm}$ wide with a $17 \mathrm{~mm}$ free length and the input was a 1.22 V step.

\begin{tabular}{|r|r|r|r|}
\hline sample & blocked force $(\mathrm{mN})$ & free deflection $(\mathrm{mm})$ & energy density $(\mathrm{mJ} / \mathrm{kg})$ \\
\hline 1 & 0.285 & 1.518 & 6.949 \\
\hline 2 & 0.135 & 1.424 & 3.088 \\
\hline 3 & 0.284 & 1.121 & 5.113 \\
\hline 4 & 0.229 & 0.957 & 3.520 \\
\hline 5 & 0.220 & 1.100 & 3.887 \\
\hline
\end{tabular}

Based on the work by Newbury and Leo, the force/deflection curves were assumed to be linear and were therefore constructed by measuring only the limiting cases of zero deflection from the neutral position and zero force generated at the tip. The useful peak energy available at the tip of a cantilevered sample is then the area under the peak force/delfection curve, which is half of the product of the peak blocked force and peak free deflection. Using equation 4.1, the mass energy densities of several samples of Nafion ${ }^{\mathrm{TM}}$ plated using the current method were measured, in order to characterize this material and compare it to the material supplied by Dr. Shahinpoor. Please refer to Table 4.3 for the measured peak blocked force, peak free deflection, and corresponding peak energy density of five strips of Nafion $^{\mathrm{TM}}-117$ that were plated with a copper/platinum alloy and a gold overlayer using the current method. These samples were $5 \mathrm{~mm}$ wide with a $17 \mathrm{~mm}$ free length and were in the lithium ion form. For an applied step input of $1.22 \mathrm{~V}$, the average peak energy density of these samples is $4.511 \mathrm{~mJ} / \mathrm{kg}$. As mentioned above, Newbury and Leo have measured the energy density of identical strips of the baseline material to be between $3.10 \mathrm{~mJ} / \mathrm{kg}$ and $11.52 \mathrm{~mJ} / \mathrm{kg}$. Therefore, the energy density of the new samples compares well with the baseline material. A similar characterization was performed on five strips of Nafion ${ }^{\mathrm{TM}}-115$ plated using the current method. As with the other samples, these strips were $5 \mathrm{~mm}$ wide with a $17 \mathrm{~mm}$ free length and were in the lithium ion form. Refer to Table 4.4 for the peak blocked force, peak free deflection, and peak energy density results. In this case some of the tests were performed twice and the two runs averaged in order to reduce the effects of random error in the data. For an applied input of $1.22 \mathrm{~V}$, the average energy density of these samples is $20.113 \mathrm{~mJ} / \mathrm{kg}$, which is much higher than the measured energy density for the baseline material or the samples of Nafion ${ }^{\mathrm{TM}}-117$ plated using the current process. The large difference in these metrics highlights the fact that the fabrication process for these 
Table 4.4: Measured peak blocked force, peak free deflection, and resulting peak energy densities of five strips of Nafion ${ }^{\mathrm{TM}}-115$ plated using the new process. Each strip was $5 \mathrm{~mm}$ wide with a $17 \mathrm{~mm}$ free length and the input was a 1.22 V step.

\begin{tabular}{|r|r|r|r|r|r|r|r|}
\hline \multirow{2}{*}{ sample } & \multicolumn{3}{|c|}{ blocked force $(\mathrm{mN})$} & \multicolumn{3}{c|}{ free deflection $(\mathrm{mm})$} & \\
\cline { 2 - 7 } & trial 1 & trial 2 & average & trial 1 & trial 2 & average & energy density $(\mathrm{mJ} / \mathrm{kg})$ \\
\hline 1 & 0.271 & 0.284 & 0.277 & 2.310 & 2.101 & 2.205 & 13.338 \\
\hline 2 & 0.310 & 0.280 & 0.295 & 2.413 & 2.101 & 2.257 & 14.501 \\
\hline 3 & 0.391 & 0.348 & 0.370 & 3.106 & 2.726 & 2.916 & 23.491 \\
\hline 4 & 0.336 & 0.327 & 0.331 & 3.001 & - & 3.001 & 21.684 \\
\hline 5 & 0.421 & 0.400 & 0.410 & 3.081 & - & 3.081 & 27.549 \\
\hline
\end{tabular}

transducers may not be well controlled and thus inconsistencies in the measured properties are often observed.

Another property of these trandsducers that was measured is their elastic modulus. The elastic or Young's modulus of a material defines how stress and strain relate to each other in a material and is important in defining models to predict electromechanical behavior. The elastic modulus was measured as described in Chapter 2, by pulling strips of plated Nafion ${ }^{\mathrm{TM}}$ between two clamps and measuring the force in the material and the amount of deflection in the material. From this data and the geometry of the sample, the stress and strain were calculated. As with the measurement of density, the nominal thickness of the membrane was used as the thickness of the samples. The elastic modulus is then the slope of the stress/strain curve. It should be pointed out that like all polymers, Nafion $^{\mathrm{TM}}$ is a viscoelastic material. In the current test, creep and relaxation effects in the polymer were neglected and the modulus data reported here should be considered to be essentially a steady-state or DC value. Refer to Table 4.5 for the measured elastic modulus of several samples of Nafion ${ }^{\mathrm{TM}_{-}}-117$ and Nafion ${ }^{\mathrm{TM}}-115$ that were plated using the current method. The average modulus for the samples of Nafion ${ }^{\mathrm{TM}}-117$ is $52.40 \mathrm{MPa}$ and the average modulus for the samples of Nafion ${ }^{\mathrm{TM}}-115$ is $61.41 \mathrm{MPa}$. Note that there is a lot of scatter in the data, with some of the measured values differing from the average by as much as $11.9 \%$. There are several possible reasons for these discrepancies, with the most likely being inconsistencies in the Nafion ${ }^{\mathrm{TM}}$ membranes or the metallic electrodes, errors in the measurement of the sample geometry, slight changes in the orientation of the samples as clamped in the fixture, and inconsistencies in the applied strain rates during the test. Also, DuPont reports the tensile elastic modulus of bare Nafion ${ }^{\mathrm{TM}}$ that has been hydrated by 
Table 4.5: Measured elastic modulus of several strips of Nafion ${ }^{\mathrm{TM}}-117$ and Nafion $^{\text {TM }}-115$ plated using the current method. All data are in MPa.

\begin{tabular}{|r|r|}
\hline Nafion $^{\mathrm{TM}}-117$ & Nafion $^{\mathrm{TM}}-115$ \\
\hline 46.17 & 54.10 \\
\hline 61.95 & 61.22 \\
\hline 57.17 & 58.01 \\
\hline 49.92 & 66.85 \\
\hline 46.81 & 66.89 \\
\hline
\end{tabular}

boiling in water to be $64 \mathrm{MPa}$ (11). It is interesting to note that this value is larger than the measured modulus of the new materials, which should be stiffer than the bare polymer due to the addition of the metal electrodes. Some possible reasons for this discrepancy are differences in the way that the tests were performed and differences in the mobile cations that were used.

Although there is quite a spread in the data, the moduli of the samples of Nafion ${ }^{\mathrm{TM}}$ 115 are consistently higher than for the samples of Nafion ${ }^{\mathrm{TM}_{-}}$117. A reasonable explanation for this trend is the same one used to explain the differences in the densities of the two batches of material. Because the two samples were processed in the same way, the electrodes in both cases are likely very similar. These electrodes would occupy a larger percentage of the total volume in the thinner membrane and have a higher modulus than the polymer, owing to the higher measured modulus of the samples of Nafion ${ }^{\mathrm{TM}}-115$. Parrot, Nicoliasen, and Leo have studied the elastic modulus of the baseline material supplied by Dr. Shahinpoor using a similar method to the one described and have found it to be approximately $190 \mathrm{MPa}$ (45). As with the density measurements, the reason for the large discrepancy between the measured elastic modulus of the new materials and the baseline material is that the baseline material contains larger amounts of metal as the electrodes. Because the modulus of the baseline material is larger, samples of this material typically generate more blocked force for a given voltage input than samples of the new material, while samples of the new material generally exhibit larger free deflections.

\subsection{Summary}

In this chapter, the theory and process behind a new method for plating metal electrodes onto Nafion $^{\mathrm{TM}}$ membranes has been presented, including experimental results and perfor- 
mance data. These results provide evidence that multiple metals can be simulataneously plated onto Nafion ${ }^{\mathrm{TM}}$ membranes using an impregnation/reduction process. The results also indicate that the stability of non-precious metals in these electrodes can be improved by alloying the electrodes with precious metals. Longevity testing of samples that were plated with a copper/platinum alloy indicate that increasing the amount of platinum present in the membrane will improve the stability of the electrodes and that the addition of a gold layer over the copper/platinum alloy electrode will improve the stability further.

The results with this new process indicate that the relative amounts of different metals in the polymer can be affected by changing the relative proportions of the metals in the solution used to perform the ion exchange and by changing the soak time of the membrane in the solution. This supports the results of work by other researchers to characterize the ion exchange properties of Nafion ${ }^{\mathrm{TM}}$ membranes. Experimental findings indicate that stable electrodes are obtained when the membranes are plated using a solution of $0.0005 \mathrm{M}$ $\left[\mathrm{Pt}\left(\mathrm{NH}_{3}\right)\right] \mathrm{Cl}_{2}+0.0095 \mathrm{M} \mathrm{CuSO}_{4}$ with subsequent electroless plating of gold.

Characterization of these new materials indicates that the mass energy density compares well to that of a baseline material obtained from Dr. Mohsen Shahinpoor of the University of New Mexico. Measurement of the elastic modulus reveals that these new materials are more compliant than the baseline material. Measurements of the blocked force and free deflection show that the new material generates less force and more deflection for a given input than the baseline material, which is what one would expect given its lower modulus. Also, the new material is found to have a density that is approximately $2 / 3$ of the density of the baseline material. The overall effect of the differences in the blocked force, free deflection, and mass density of these two materials is that the energy densities of the two materials are very similar.

Testing of the sensing capability of the new materials reveals that the coupling between strain rate and short-circuit current is lower than in the baseline material. Some possible reasons for this difference are the different ways that this metric was obtained in the two materials and differences in the mechanical properties of the two materials, although at this time a causal relationship has not been determined.

The results of the tests performed on the devices made using the new process has indicated that they seem to perform well with regard to acheivable forces and deflections as well as continuous operation with little degredation of performance. Discrepancies and 


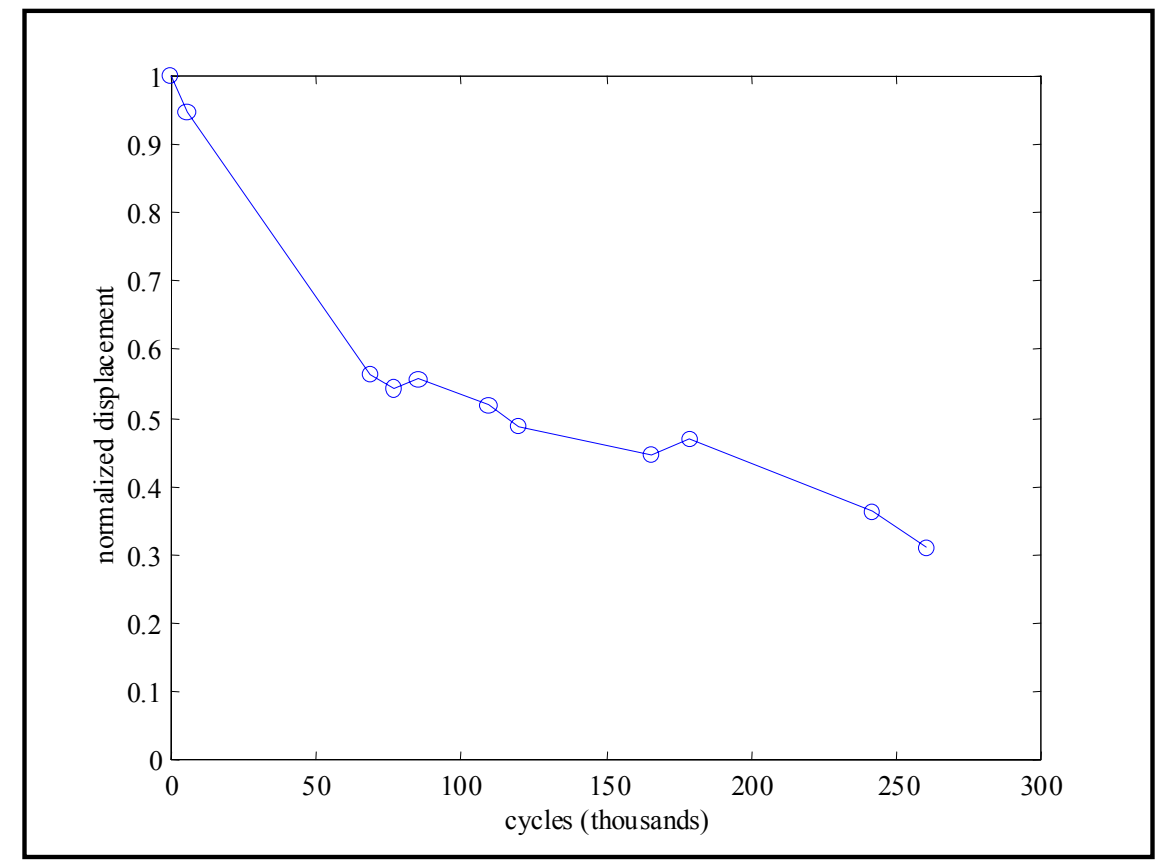

Figure 4.13: Normalized tip displacement versus the number of actuation cycles for a sample of Nafion ${ }^{\mathrm{TM}}-117$ plated using the new process. The input was a $1.25 \mathrm{~V}, 1.0 \mathrm{~Hz}$ sine wave.

scatter in the measured data reveal that inconsistencies in the processing still exists, however. To highlight this fact, refer to Figure 4.13. This figure shows a longevity result of a Nafion ${ }^{\mathrm{TM}}$ actuator treated using the new process and actuated continuously for over 250,000 cycles. This test was meant to confirm the results obtained in Figure 4.11, although degredation of the free tip deflection is more evident in this case, as can be seen from the figures. Using this new plating process, the stability of the metal electrodes has been significantly improved over that of the electrodes manufactured using the processes described in Chapter 3, but further work should be performed in order to optimize the process for indefinite actuator life with minimal noble metal loading. Improvements to the processing technique should also reduce the occurences of inconsistencies in the measured properties of these devices. 


\section{Chapter 5}

\section{Conclusions}

This thesis has presented the results of a study aimed at reducing the cost of electrodes on ionic polymer transducers. During this work, several different methods of plating metal electrodes onto Nafion ${ }^{\mathrm{TM}}$ membranes have been studied and analyzed for their effectiveness in making a good ionic polymer actuator and sensor. The critical characteristics of these electrodes have been identified and a new process for fabricating the electrodes has been developed. Ionic polymer devices made using this process have been tested and compared to a baseline material. Results indicate that the transducers made using the new process perform as well as the baseline material but have electrodes containing lower amounts of noble metal. Specific conclusions of this research are outlined in the following section.

\subsection{Key Results and Conclusions}

Based on the initial work with several different plating processes, several key features of the electrodes were identified. Samples of Nafion ${ }^{\mathrm{TM}}$ were plated with metal in processes resulting in interlocking of the polymer and the metal phases as well as in processes involving purely surface deposition. This interlocking was found to affect the adhesion of the electrode to the polymer as well as the electromechanical transduction properties of the device. Using the gold sputter-coating process and the electroless nickel plating process, there is not interpenetration of the metal into the polymer. The work with these two prcesses revealed that adhesion of the electrode to the polymer membrane was problematic. These electrodes were found to flake or rub off of the membrane during actuation or handling of the samples. Efforts to etch or roughen the surfaces of the membrane prior to plating 
had little effect on this adhesion problem. Another plating process was investigated that did involve penetration of the electrode into the polymer membrane. This process involves exchange of metal ions into the membrane followed by reduction of the ions to metallic state at the surface of the membrane. Adhesion of the electrode in this case was not a problem.

Also improved by the incorporation of interlocking electrodes was the actuation performance of the devices. Initial results with surface plating indicated that although electromechanical transduction with these devices was evident, the coupling in these devices was lower than in the baseline material. Results with the new process indicate that the new materials perform at least as well as the baseline material. The reason for the difference in the performance of the two types of actuators is that the actuators with interlocking electrodes experience a much higher charge density at the membrane/electrode interface when activated by a voltage. Because the polymer/metal interfacial area is higher in this case, the electric field for a given actuation voltage is higher, and the electrostatic force acting on the free cations in the membrane is higher.

Through these various plating methods, several different metals were also investigated as electrodes, including both precious and non-precious metals. The key issue with the use of non-precious metals in the electrodes of ionic polymer membrane devices has been identified as oxidation of the metal. This oxidation is not present in samples made using platinum electrodes or in the baseline material, which uses gold as the electrode. When nickel or copper is used as the electrode metal, oxidation is rapid and has extremely detrimental effects on the actuation performance of the device. This work has shown that the oxidation of non-precious metals in the electrode can be affected by the addition of precious metals as alloys. Further, experimental results demonstrate that the stability of the electrode is a direct function of the content of precious metal. Also, addition of a protective layer of precious metal over this initial alloy layer further improves the stability, allowing for stable sublayers containing lesser amounts of precious metal.

These precious/non-precious metal alloy layers are fabricated by simulataneously exchanging a precious and non-precious metal ion into the membrane and subsequently reducing these ions to metallic state at the surface of the membrane, resulting in a composite electrode that exists a few microns deep in the polymer. Studies were performed in order to determine the effect of the exchanging solution and the soak time of the membrane in the solution on the composition of the resulting electrode. The results of these studies reveals 
that the copper and tetraammine platinum ions (both of charge +2 ) behave in the way predicted by the results of Yeager and Steck $(54 ; 65 ; 63)$. That is, for ions of the same charge, larger ions will generally have higher selectivity coefficients (because they are less solvated) and smaller self-diffusion coefficients (because of their larger size).

As mentioned, the new plating process typically deposits metal electrodes to depths of 2-3 microns in the Nafion ${ }^{\mathrm{TM}}$ membrane. This alloy electrode is then covered with a protective gold layer that is on the order of 50 nanometers thick. Scanning electron micrographs of the baseline material reveals that it contains gold electrodes that penetrate into the membrane to depths of up to 15 microns and that an additional gold surface layer is deposited that is about 250 nanometers thick. Previous testing has shown that the mass energy density of the baseline material is on the order of $3-11 \mathrm{~mJ} / \mathrm{kg}$. Results with the new material indicate that its energy density ranges from 4 to $20 \mathrm{~mJ} / \mathrm{kg}$, which compares well to the baseline material. This indicates that ionic polymer actuators can be made using significantly less amounts of noble metal without sacrifices to the performance of these materials. Testing has also confirmed that because of the thinner electrodes, the elastic modulus and mass density of these materials is much lower than the baseline material. Thus although the new materials generate more free deflection but less blocked force than the baseline material, the mass energy density is similar to the baseline.

\subsection{Contributions}

During the course of this research project, several different methods for plating metal onto ionic polymers were developed and studied, including processes involving purely surface deposition of metals and methods involving interpenetration of the metal and the polymer. Several different metals were also plated as electrodes and the issues associated with the use of each of these metals and methods were analyzed. Based on these findings, a new process has been developed that involves significant reductions in the amount of noble metal required for deposition of stable electrodes, and ionic polymer transducers can be manufactured using this new processing method in less than five hours. Testing of these new materials indicates that their properties compare favorably with the baseline material supplied by Dr. Mohsen Shahinpoor of the University of New Mexico. 


\subsection{Suggestions for Future Work}

Although significant advancements to the processes for fabricating ionic polymer membrane actuators and sensors have been made in this study, there are still many aspects of research in this field that require attention. Some possible areas of future research include:

- Further improvements to the new plating process including studies into how various aspects of the electrodes and the performance of devices are affected by changes to the process and simulated and experimental optimizations of the process parameters. The process should be improved to the point that the amount of noble metal used is minimized and the resulting devices have indefinite life under actuation conditions. Efforts should also focus on improving the consistency of the devices made using the process by identifying and eliminating the major sources of the inconsistencies.

- Studies of other types of electrode materials would be useful. It is probable that other types of alloys may work as well or better than copper/platinum as far as actuation performance and stability of the electrode are concerned. Conductive oxides may also be effective as stable electrodes.

- In this study, only Nafion ${ }^{\mathrm{TM}}$ membranes were used, but many other types of ionic polymer membranes exist or are being developed. An understanding of the features of the polymer that benefit its electromechanical transduction would allow new types of polymers to be designed specifically for smart material applications.

- Several researchers have determined that the effect of changing the free cation in the polymer is that both the magnitude and speed of the response is changed. To date, researchers have only used actuators containing a single cation, however. This work and work by others proves that multiple ions can be exchanged into an ionic polymer membrane simultaneously. This opens the door for a study of the effect of exchanging multiple ions in a membrane on the membrane's performance as an electromechanical transducer, including the effect on both the sensing and actuation properties.

- Other ionic polymer actuation and sensing technologies exist, including conductive polymer actuators and carbon nanotube actuators. Potential exists for incorporation of these technologies with ionic polymer actuators to make hybrid actuators that may 
combine the benefits of these technologies and offer improved performance over the current state-of-the-art.

- A fundamental issue with the use of ionic polymer membranes in commercial applications is that they must remain hydrated in order to function. The development of an effective barrier coating would allow this technology to operate in many more environments than are currently considered possible and would make it competitive with other types of smart material technologies.

- Another approach to overcome the issue of material hydration is to select ionic polymer materials that do not require hydration for ionic motion to occur. The development of such a device would solve the problem of material hydration without requiring the development of a barrier coating.

- Although they generate appreciable displacements, ionic polymer membranes are too compliant to generate useful forces. One possibility is that these materials may be stacked in multiple layers such that the large bending displacement is preserved while forces of much greater magnitude can be produced.

- Most of the research into ionic polymers as actuators has focused on using them as cantilevered benders. While this is interesting, an analysis of the capabilities of these materials in other geometries is important. Such an analysis would likely result in the development of new actuation strategies for these materials that result in much greater available energy output, this increasing the usefulness of the material. 


\section{Bibliography}

[1] Y. Abe, A. Mochizuki, T. Kawashima, S. Yamashita, K. Asaka, and K. Oguro. Effect on bending behavior of counter cation species in perfluorinated sulfonate membraneplatinum composite. Polymers for Advanced Technologies, 9:520-526, 1998.

[2] Alfa Aesar, a Johnson Matthey Company. Gold Plating Solution, Electroless, Metal Content=3.7 g/l, Stock \#4230\%. Product Bulletin.

[3] Y. Bar-Cohen and S. Leary. Electro-active polymer (EAP) characterization methods. In EAP Actuators and Devices, volume 3987, pages 12-16. SPIE, 2000.

[4] Y. Bar-Cohen, S. Leary, M. Shahinpoor, J. Harrison, and J. Smith. Flexible low-mass devices and mechanisms actuated by electroactive polymers. In EAP Actuators and Devices, volume 3669, pages 51-56. SPIE, 1999.

[5] Y. Bar-Cohen, S. Leary, M. Shahinpoor, J. O. Harrison, and J. Smith. Electro-active polymer (EAP) actuators for planetary applications. In EAP Actuators and Devices, volume 3669, pages 57-63. SPIE, 1999.

[6] G. D. Bari. Nickel Currents, volume 6. Inco Limited, Fall 1999.

[7] J. V. Cathcart. The Structure and Properties of Thin Oxide Films, chapter 2, pages 27-29. American Society for Metals, 1970.

[8] Y.-L. Chen and T.-C. Chou. Metals and alloys bonded on solid polymer electrolyte for electrochemical reduction of pure benzaldehyde without liquid supporting electrolyte. Journal of Electroanalytical Chemistry, 360:247-259, 1993.

[9] R. L. Cook, R. C. MacDuff, and A. F. Sammells. Ambient temperature gas phase $\mathrm{CO}_{2}$ 
reduction to hydrocarbons at solid polymer electrolyte cells. Journal of the Electrochemical Society, 135:1470-1471, 1988.

[10] D. W. Dewulf and A. J. Bard. The electrochemical reduction of $\mathrm{CO}_{2}$ to $\mathrm{CH}_{4}$ and $\mathrm{C}_{2} \mathrm{H}_{4}$ at $\mathrm{Cu} /$ Nafion electrodes (solid polymer electrolyte structures). Catalysis Letters, 1:73-80, 1988.

[11] DuPont. Nafion Membranes NE-112, NE-1135, N-115, N-11\%. Product Information.

[12] T. D. Gierke and W. Y. Hsu. Perfluorinated Ionomer Membranes, chapter 13, pages 283-307. American Chemical Society, 1982.

[13] A. J. Grodzinsky and J. R. Melcher. Electromechanics of deformable charged polyelectrolyte membranes. In Proceedings of the 27th Annual Conference on Engineering in Medicine and Biology, volume 16, page 485. Alliance for Engineering in Medicine and Biology, 1974. paper 53.2.

[14] A. J. Grodzinsky and J. R. Melcher. Electromechanical transduction with charged polyelectrolyte membranes. IEEE Transactions on Biomedical Engineering, BME23(6):421-433, 1976.

[15] S. Guo, T. Fukuda, N. Kato, and K. Oguro. Development of underwater microrobot using ICPF actuator. In International Conference on Robotics and Automation, pages 1829-1834. IEEE, 1998.

[16] S. Guo, T. Fukuda, K. Kosuge, F. Arai, K. Oguro, and M. Negoro. Micro catheter system with active guide wire. In International Conference on Robotics and Automation, pages 79-84. IEEE, 1995.

[17] R. H. Hamlen, C. E. Kent, and S. N. Shafer. Electrolytically activated contractile polymer. Nature, 206:1149-1150, 1965.

[18] A. Katchalsky. Rapid swelling and deswelling of reversible gels of polymeric acids by ionization. Experientia, 5:318-319, 1948.

[19] A. Keshavarzi, M. Shahinpoor, K. J. Kim, and J. Lantz. Blood pressure, pulse rate, and rhythm measurement using ionic polymer-metal composites sensors. In EAP Actuators and Devices, volume 3669, pages 369-376. SPIE, 1999. 
[20] K. J. Kim and M. Shahinpoor. The synthesis of nano-scaled platinum particles (NSPP)their role in performance improvement of ionic polymer-metal composite (IPMC) artificial muscles. In EAP Actuators and Devices, volume 4329, pages 189-198. SPIE, 2001.

[21] W. Kuhn. Reversible dehung und kontraktion bei anderung der ionisation eines netzwerks polyvalenter fadenmolekulionen. Experientia, 5:318-319, 1948.

[22] W. Kuhn, B. Hargitay, A. Katchalsky, and H. Eisenberg. Reversible dialation and contraction by changing the state of high-polymer acid networks. Nature, 165:514-516, 1950.

[23] R. J. Lawrance and L. D. Wood. Method of making solid polymer electrolyte catalytic electrodes and electrodes made thereby. U.S. Patent 4,272,353, 1981.

[24] M. Le Guilly, M. Uchida, and M. Taya. Nafion based smart membrane as an actuator array. In EAP Actuators and Devices, volume 4695. SPIE, 2002. to appear.

[25] R. Liu, W.-H. Her, and P. Fedkiw. In situ electrode formation on a nafion membrane by chemical platinization. Journal of the Electrochemical Society, 139(1):15-23, 1992.

[26] Y. Liu, T. Zeng, Y.-X. Wang, H. Yu, , and R. Claus. Self-assembled flexible electrodes on electroactive polymer actuators. In Electroactive Polymer Actuators and Devices, volume 3669, pages 284-288. SPIE, 1999.

[27] K. Mallavarapu and D. J. Leo. Feedback control of resonant modes in bending response of ionic polymer actuators. In International Adaptive Structures and Materials Systems Symposium. ASME, 2001. to appear.

[28] K. Mallavarapu, K. Newbury, and D. J. Leo. Feedback control of the bending response of ionic polymer-metal composites actuators. In EAP Actuators and Devices, volume 4329, pages 301-310. SPIE, 2001.

[29] P. Millet. Noble metal-membrane composites for electrochemical applications. Journal of Chemical Education, 76(1):47-49, 1999. 
[30] P. Millet, F. Andolfatto, and R. Durand. Preparation of solid polymer electrolyte composites: Investigation of the precipitation process. Journal of Applied Electrochemistry, 25:233-239, 1995.

[31] P. Millet, F. Andolfatto, and R. Durand. Preparation of solid polymer electrolyte composites: Investigation of the ion-exchange process. Journal of Applied Electrochemistry, 25:227-232, 1995.

[32] P. Millet, R. Durand, E. Dartyge, G. Tourillon, and A. Fontaine. Precipitation of metallic platinum into nafion ionomer membranes. Journal of the Electrochemical Society, 140(5):1373-1379, 1993.

[33] P. Millet, M. Pineri, and R. Durand. New solid polymer electrolyte composites for water electrolysis. Journal of Applied Electrochemistry, 19:162-166, 1989.

[34] M. Mojarrad and M. Shahinpoor. Ion-exchange-metal composite sensor films. In Smart Materials and Structures, volume 3042, pages 52-60. SPIE, 1997.

[35] S. Nemat-Nasser and J. Y. Li. Electromechanical response of ionic polymer-metal composites. Journal of Applied Physics, 87(7):3321-3331, 2000.

[36] K. Newbury and D. Leo. Electromechanical modeling and characterization of ionic polymer benders. In 12th International Conference on Adaptive Structures and Technologies, 2001. to appear.

[37] K. Newbury and D. J. Leo. Mechanical work and electromechanical coupling in ionic polymer bender actuators. In Proceedings of the ASME International Mechanical Engineering Conference and Exposition. ASME, 2001. paper AD-23705.

[38] Z. Ogumi, T. Kuroe, and Z. ichiro Takehara. Gas permeation in spe method ii. oxygen and hydrogen permeation through nafion. Journal of the Electrochemical Society, 132(11):2601-2605, 1985.

[39] K. Oguro. Actuator element. U.S. Patent 5,268,082, 1993.

[40] K. Oguro. Preparation Procedure Ion-Exchange Polymer Metal Composites (IPMC) Membranes. Osaka National Research Institute, AIST, Japan, 2001. URL: http://ndeaa.jpl.nasa.gov/nasa-nde/lommas/eap/IPMC_PrepProcedure.htm. 
[41] K. Oguro, N. Fujiwara, K. Asaka, K. Onishi, and S. Sewa. Polymer electrolyte actuator with gold electrodes. In EAP Actuators and Devices, volume 3669, pages 64-71. SPIE, 1999.

[42] K. Oguro, Y. Kawami, and H. Takenaka. Bending of an ion-conducting polymer filmelectrode composite by an electric stimulus at low volage. Journal of Micromachine Society, 5:27-30, 1992.

[43] K. Onishi, S. Sewa, K. Asaka, N. Fujiwara, and K. Oguro. Bending response of polymer electrolyte actuator. In EAP Actuators and Devices, volume 3987, pages 121-128. SPIE, 2000.

[44] K. Onishi, S. Sewa, K. Asaka, N. Fujiwara, and K. Oguro. Bending response of polymer electrolyte actuator. In EAP Actuators and Devices, volume 3987, pages 121-128. SPIE, 2000.

[45] O. Parrott, A. Nicolaisen, and D. Leo. Extensional transduction properties of ionic polymer materials. In EAP Actuators and Devices. SPIE, 2002. to appear.

[46] T. Rashid and M. Shahinpoor. Force optimization of ionic polymermic platinum composite artificial muscles by means of orthogonal array manufacturing method. In EAP Actuators and Devices, volume 3669, pages 289-298. SPIE, 1999.

[47] K. Sadeghipour, R. Salomon, and S. Neogi. Development of a novel electrochemically active membrane and 'smart' material based vibration sensor/damper. Smart Materials and Structures, 1:172-179, 1992.

[48] D. Segalman, W. Witkowski, D. Adolf, and M. Shahinpoor. Theory of electrically controlled polymeric muscles as active materials in adaptive structures. Smart Materials and Structures, 1:44-54, 1992.

[49] M. Shahinpoor, Y. Bar-Cohen, J. Simpson, and J. Smith. Ionic polymer-metal composites (IPMCs) as biomimetic sensors, actuators and artificial muscles - a review. Smart Materials and Structures, 7(6):R15-R30, 1998.

[50] M. Shahinpoor and K. Kim. Ionic polymer-metal composites: I. fundamentals. Smart Materials and Structures, 10:819-833, 2001. 
[51] M. Shahinpoor and K. Kim. Novel physically loaded and interlocked electrode developed for ionic polymer-metal composites (IPMC's). In Electroactive Polymer Actuators and Devices, volume 4329, pages 174-181. SPIE, 2001.

[52] M. Shahinpoor and K. J. Kim. Effect of counter-ions on the performance of IPMCs. In EAP Actuators and Devices, volume 3987, pages 110-120. SPIE, 2000.

[53] M. Shahinpoor and K. J. Kim. The effect of surface-electrode resistance on the performance of ionic-polymer metal composite (IPMC) artificial muscles. Smart Materials and Structures, 9:543-551, 2000.

[54] A. Steck and H. L. Yeager. Water sorption and cation-exchange selectivity of a perfluorosulfonate ion-exchange polymer. Analytical Chemistry, 52(8):1215-1218, 1980.

[55] S. Surampudi, H. A. Frank, S. R. Narayanan, W. Chun, B. Jeffries-Nakamura, A. Kindler, and G. Halpert. Direct methanol feed fuel cell and system. U.S. Patent 6,265,093, 2001.

[56] S. Surampudi, H. A. Frank, S. R. Narayanan, W. Chun, B. Jeffries-Nakamura, A. Kindler, and G. Halpert. Direct methanol feed fuel cell and system. U.S. Patent $6,254,748,2001$.

[57] S. Tadokoro, T. Murakami, S. Fuji, R. Kanno, M. Hattori, and T. Takamori. An elliptical friction drive element using an ICPF (ion conducting polymer gel film) actuator. In International Conference on Robotics and Automation, pages 205-212. IEEE, 1996.

[58] H. Takenaka and E. Torikai. Japanese Patent 5,538,934, 1980.

[59] H. Takenaka, E. Torikai, Y. Kawami, and N. Wakabayashi. Solid polymer electrolyte water electrolysis. International Journal of Hydrogen Energy, 7(5):397-403, 1982.

[60] C. D. Wagner, W. M. Riggs, L. E. Davis, and J. F. Moulder. Handbook of X-ray Photoelectron Spectroscopy. Perkin-Elmer Corporation, Physical Electronics Division, 6509 Flying Cloud Drive, Eden Prarie, Minnesota, 55344, 1979.

[61] M. S. Wilson and S. Gottesfeld. Thin-film catalyst layers for polymer electrolyte fuel cell electrodes. Journal of Applied Electrochemistry, 22:1-7, 1992. 
[62] I. V. Yannas and A. J. Grodzinsky. Electromechanical energy conversion with collagen fibers in an aqueous medium. Journal of Mechanochemistry and Cell Motility, 2:113$125,1973$.

[63] H. L. Yeager. Perfluorinated Ionomer Membranes, chapter 3-4, pages 25-63. American Chemical Society, 1982.

[64] H. L. Yeager and A. Eisenberg. Perfluorinated Ionomer Membranes, chapter 1, pages 1-6. American Chemical Society, 1982.

[65] H. L. Yeager and A. Steck. Ion-exchange selectivity and metal ion separations with a perfluorinated cation-exchange polymer. Analytical Chemistry, 51(7):862-865, 1979.

[66] H. L. Yeager and A. Steck. Cation and water diffusion in nafion ion exchange membranes: Influence of polymer structure. Journal of the Electrochemical Society, 128:1880-1884, 1981.

[67] W. Zhou, W. J. Li, N. Xi, and S. Ma. Development of force-feedback controlled nafion micromanipulators. In EAP Actuators and Devices, volume 4329, pages 401-410. SPIE, 2001. 


\section{Vita}

The author, Matthew Damon Bennett was born on July 31, 1977 to Daniel Bennett and Cheryl Gill. He grew up in South Carolina with his mother and stepfather, Robert Gill. Matthew attended high school at Riverside High School in Greer, South Carolina and was always very interested in science and engineering. He participated in numerous science fairs and in 1995 was on a team of students that took first place in the TEAMS competition, a statewide engineering competition among high school students. In the fall of 1995 Matthew left home to attend Virginia Tech, where he majored in mechanical engineering. During his time as an undergraduate, Matthew participated in many student design projects, including a project during his freshman year in which he assisted a group of seniors in the design of a mixing nozzle using MEMS technology. During the summer after his sophomore year he assisted Dr. Jan Helge Bøhn in an undergraduate project to build an aircrew oxygen mask using rapid prototyping. In his senior year Matthew served as the propulsion design crew chief on Virginia Tech's Human Powered Submarine Team. That year the team won first place in five out of eight categories at the ASME-sponsored international competition and took second place in the other three categories. Following graduation with a B.S. degree in May of 2000, Matthew enrolled in the graduate school at Virginia Tech. In the first semester of his Master's work, Matthew was a graduate teaching assistant for Mechanical Engineering Lab II, a senior-level course in the mechanical engineering department. After this Matthew studied ionic polymers under the advisement of Dr. Don Leo. After completion of his Master's work, Matthew will move on to pursue a Doctor of Philosophy under the advisement of Dr. Leo. During this work Matthew will study other aspects of ionic polymer transducers and their use. Following the completion of this degree, Matthew would like either to pursue a career in academia or to become an entrepreneur and start a company.

In his free time Matthew enjoys water skiing, snowboarding, SCUBA diving, camping, and playing guitar. He also enjoys movies and good music, especially jazz, and loves to travel. 